\title{
S3-Leitlinie „Sedierung in der gastrointestinalen Endoskopie“ 2008 (AWMF-Register-Nr. 021/014)
}

\section{S3-Guidelines - Sedation in Gastrointestinal Endoscopy}

Authors

Affiliation
A. Riphaus, T. Wehrmann, B. Weber, J. Arnold, U. Beilenhoff, H. Bitter, S. von Delius, D. Domagk, A. F. Ehlers, S. Faiss, D. Hartmann, W. Heinrichs, M.-L. Hermans, C. Hofmann, S. In der Smitten, M. Jung, G. Kähler, M. Kraus, J. Martin, A. Meining, J. Radke, T. Rösch, H. Seifert, A. Sieg, B. Wigginghaus, I. Kopp

Die Institutsangaben sind in $\bullet$ Tab. 1 des Beitrages gelistet.
Bibliography

DOI 10.1055/s-2008-1027850

Z Gastroenterol 2008; 46:

1298 - 1330 @ Georg Thieme

Verlag KG Stuttgart - New York. ISSN 0044-2771

\section{Correspondence} Dr. Andrea Riphaus

Medizinische Klinik I, Klinikum Region Hannover $\mathrm{GmbH}$, Krankenhaus Siloah Roesebeckstr. 15

30449 Hannover

Tel.: ++ 49/511/9272231

Fax: ++49/511/9272669

ariphaus@web.de

\section{Prof. Dr. Till Wehrmann}

Fachbereich Gastroenterologie, Deutsche Klinik für Diagnostik Aukammallee 33

65193 Wiesbaden

Tel.: ++ 49/611/57 7212

Fax: $++49 / 611 / 577460$

till.wehrmann@dkd-

wiesbaden.de
Herausgeber der Leitlinie ist die Sektion Endoskopie im Auftrag der Deutschen Gesellschaft für Verdauungs- und Stoffwechselerkrankungen e.V. (DGVS), die auch die federführende Fachgesellschaft ist. Mitherausgeber sind die an der Leitlinienentwicklung beteiligten Fachgesellschaften und Organisationen:

- Bundesverband Niedergelassener Gastroenterologen Deuschlands e.V. (Bng)

- Chirurgischen Arbeitsgemeinschaft für Endoskopie und Sonographie der Deutschen Gesellschaft für Allgemein- und Viszeralchirurgie (DGAV)

- Deutsche Morbus Crohn/Colitis ulcerosa Vereinigung e.V. (DCCV)

- Deutsche Gesellschaft für Endoskopie-Assistenzpersonal (DEGEA)

- Deutsche Gesellschaft für Anästhesie und Intensivmedizin (DGAI)

- Gesellschaft für Recht und Politik im Gesundheitswesen (GPRG)

Unter der Leitung von: T. Wehrmann, A. Riphaus, I. Kopp 


\section{Inhaltsverzeichnis}

\begin{tabular}{|c|c|c|}
\hline & Einleitung & 1300 \\
\hline 1. & $\begin{array}{l}\text { Themenkomplex I: Indikationen/Ziele/be- } \\
\text { kannte Risiken/Patienten/Qualitätsziele }\end{array}$ & 1305 \\
\hline 1.1. & Empfehlung Sedierungsangebot & 1305 \\
\hline 1.2 & $\begin{array}{l}\text { Empfehlung zur Indikationsstellung für eine } \\
\text { Sedierung }\end{array}$ & 1305 \\
\hline 1.3. & Empfehlung Untersuchungsqualität & 1305 \\
\hline 1.4. & $\begin{array}{l}\text { Empfehlung Risikoabschätzung und Struk- } \\
\text { turqualität }\end{array}$ & 1306 \\
\hline 1.5. & Empfehlung Anästhesie/Intubation & 1307 \\
\hline 1.6. & Empfehlung Schutzintubation & 1307 \\
\hline 1.7. & Empfehlung Lagerung & 1308 \\
\hline 2. & $\begin{array}{l}\text { Themenkomplex II: Sedativa/Analgetika/ve- } \\
\text { getativ wirksame Pharmaka/Kombinations- } \\
\text { therapie/Applikationstechniken }\end{array}$ & 1308 \\
\hline 2.1 . & Akzeptanz Patient und Untersucher & 1308 \\
\hline 2.1.1. & Patientenakzeptanz/Zufriedenheit & 1308 \\
\hline 2.1.2. & Untersucherzufriedenheit & 1308 \\
\hline 2.2 & Monotherapien & 1309 \\
\hline 2.2.1. & Propofol & 1309 \\
\hline 2.2.1.1. & Allgemeines & 1309 \\
\hline 2.2.1.2. & Techniken der Propofol-Applikation & 1310 \\
\hline 2.2.1.2.1. & Intermittierende Propofol-Bolusapplikation & 1310 \\
\hline 2.2.1.2.2. & $\begin{array}{l}\text { Kontinuierliche Propofol-Applikation mittels } \\
\text { Perfusorsystemen }\end{array}$ & 1310 \\
\hline 2.2.1.2.3. & $\begin{array}{l}\text { Patientenkontrollierte (Analgo)-Sedierung } \\
\text { (PCS) }\end{array}$ & 1310 \\
\hline 2.2.1.2.4. & Target-Controlled Infusion (TCI) & 1310 \\
\hline 2.2.1.2.5. & $\begin{array}{l}\text { Computer-Assisted Personalized Sedation } \\
\text { (CAPS) }\end{array}$ & 1311 \\
\hline 2.2.2. & Benzodiazepine & 1311 \\
\hline 2.2.2.1. & Diazepam & 1311 \\
\hline 2.2.2.2. & Midazolam & 1311 \\
\hline 2.2.2.2.1. & $\begin{array}{l}\text { Antagonisierung der Wirkung von Midazolam } \\
\text { durch Flumazenil }\end{array}$ & 1312 \\
\hline 2.2.2.3. & Amnesie - Diazepam vs. Midazolam & 1312 \\
\hline 2.2.3. & Propofol versus Midazolam & 1312 \\
\hline 2.2.3.1. & Kardiorespiratorische Komplikationen & 1312 \\
\hline 2.2.4. & Sonstige Pharmaka als Monotherapeutika & 1313 \\
\hline 2.2.4.1. & Einleitung & 1313 \\
\hline 2.2.4.2. & Opiate als Monotherapeutikum & 1313 \\
\hline 2.2.4.2.1. & Fentanyl & 1313 \\
\hline 2.2.4.2.1.2. & Allgemeines & 1313 \\
\hline 2.2.4.2.2 & Remifentanil & 1314 \\
\hline 2.2.4.2.2.1. & Allgemeines & 1314 \\
\hline 2.2.4.3. & Ketamin als Monotherapeutikum & 1314 \\
\hline 2.2.4.3.1. & Allgemeines & 1314 \\
\hline 2.2.4.4 & $\begin{array}{l}\text { Inhalationsanästhetika als Monotherapeuti- } \\
\text { kum }\end{array}$ & 1314 \\
\hline 2.2.4.4.1. & Distickstoffmonoxid (Lachgas) & 1314 \\
\hline 2.2.4.4.1.2. & Allgemeines & 1314 \\
\hline 2.2.4.5 & Nicht steroidale Antiphlogistika & 1315 \\
\hline 2.2.4.5.1. & Keterolac trometamol & 1315 \\
\hline 2.2.4.5.1.2. & Allgemeines & 1315 \\
\hline 2.3 . & Kombinationstherapien & 1315 \\
\hline 2.3.1. & Allgemeines & 1315 \\
\hline 2.3.2. & Spezifische Kombinationen & 1315 \\
\hline 2.3.2.1. & Kombination Sedativum plus Opiat & 1315 \\
\hline 2.3.2.2. & Kombination Sedativum mit Propofol & 1316 \\
\hline 2.3.2.3. & Kombination Sedativum plus Spasmolytikum & 1316 \\
\hline 2.3.3. & Nebenwirkungen bei Kombinationstherapie & 1316 \\
\hline 2.3.3.1. & Allgemeines & 1316 \\
\hline 2.3.4. & Monitoring/Strukturqualität & 1316 \\
\hline 2.4 . & Einfluss der Ko-Morbidität & 1317 \\
\hline
\end{tabular}

\begin{tabular}{|c|c|c|}
\hline 2.4.1. & Allgemeines & 1317 \\
\hline 2.4.2. & Risikopatienten & 1317 \\
\hline 2.4.3. & Substanzart & 1317 \\
\hline 2.5 . & Musik in der Endoskopie & 1318 \\
\hline 3. & $\begin{array}{l}\text { Themenkomplex III: Strukturqualität persönli- } \\
\text { che/personelle/apparative Voraussetzungen }\end{array}$ & 1318 \\
\hline 3.1. & Persönliche Voraussetzungen & 1318 \\
\hline 3.2. & Ausbildungs- und Trainingskurse & 1318 \\
\hline 3.3. & Personelle Voraussetzungen & 1319 \\
\hline 3.3.1. & Ausbildungsvoraussetzungen & 1319 \\
\hline 3.3.2. & Überwachung der Sedierung & 1319 \\
\hline 3.3.3. & Durchführung der Sedierung & 1319 \\
\hline 3.3.3.1. & $\begin{array}{l}\text { Voraussetzungen bei durch Pflegepersonal } \\
\text { verabreichter Propofol-Sedierung (sog. „Nur- } \\
\text { se-administered propofol sedation [NAPS]“) }\end{array}$ & 1319 \\
\hline 3.3.3.2. & $\begin{array}{l}\text { Einschränkungen für die durch Pflegepersonal } \\
\text { verabreichte Propofol-Sedierung („NAPS“) }\end{array}$ & 1319 \\
\hline 3.3.4. & Überwachung nach der Endoskopie & 1319 \\
\hline 3.4 & Räumliche Ausstattung & 1320 \\
\hline 3.4.1 & Allgemeines & 1320 \\
\hline 3.5. & Apparative Ausstattung & 1320 \\
\hline 3.5.1. & Klinische Überwachung/Standard-Monitoring & 1320 \\
\hline 3.5.2. & Erweitertes Monitoring & 1320 \\
\hline 3.5.2.1. & Kapnografie & 1320 \\
\hline 3.5.2.2. & Monitoring mittels EEG & 1320 \\
\hline 4. & $\begin{array}{l}\text { Themenkomplex IV: Prozessqualität Aufklä- } \\
\text { rung, Einwilligung/Voraussetzung für Durch- } \\
\text { führung der Sedierung/Sicherung vitaler } \\
\text { Funktionen/klinische Überwachung/Zwi- } \\
\text { schenfallmanagement }\end{array}$ & 1321 \\
\hline 4.1. & Patientenaufklärung und -einwilligung & 1321 \\
\hline 4.1.1. & Allgemeine und rechtliche Aspekte & 1321 \\
\hline 4.1.2. & Aufklärende Person & 1321 \\
\hline 4.1.3. & Durchführung der Aufklärung & 1321 \\
\hline 4.1.4. & Inhalte des Aufklärungsgesprächs & 1321 \\
\hline 4.1.5. & $\begin{array}{l}\text { Sicherungsaufklärung (Verhalten nach der } \\
\text { Sedierung) }\end{array}$ & 1322 \\
\hline 4.2 . & $\begin{array}{l}\text { Voraussetzung für die Durchführung der Se- } \\
\text { dierung }\end{array}$ & 1322 \\
\hline 4.3. & Sicherung vitaler Funktionen & 1322 \\
\hline 4.4 . & Zwischenfallmanagement & 1322 \\
\hline 4.4.1. & Hypoxie & 1322 \\
\hline 4.4.2. & Herzrhythmusstörungen & 1323 \\
\hline 4.4.2.1. & Allgemeines & 1323 \\
\hline 4.4.2.2. & Tachykarde Herzrhythmusstörungen & 1323 \\
\hline 4.4.2.3. & Bradykarde Herzrhythmusstörungen & 1323 \\
\hline 4.4.3. & Arterielle Hypotonien & 1323 \\
\hline 4.4.4. & Myokardiale Ischämien & 1323 \\
\hline 4.4.5. & Seltene Ereignisse bei Sedierung & 1323 \\
\hline 5. & $\begin{array}{l}\text { Themenkomplex V: Ergebnisqualität durch } \\
\text { Qualitätsziele/interne Qualitätssicherung/Ent- } \\
\text { lassungskriterien/„Street-Fitness“/Arbeitsfä- } \\
\text { higkeit/Dokumentation/„Benchmarking“ }\end{array}$ & 1323 \\
\hline 5.1. & Interne Qualitätssicherung & 1323 \\
\hline 5.2 & Entlassungskriterien & 1324 \\
\hline 5.2.1. & Patienteninstruktionen & 1324 \\
\hline 5.2.2. & Minimalkriterien für die Entlassung & 1324 \\
\hline 5.2.3. & $\begin{array}{l}\text { Verwendung von Score-Systemen für die Ent- } \\
\text { lassung }\end{array}$ & 1324 \\
\hline 5.3 & „Street-Fitness" & 1324 \\
\hline 5.3.1. & Verkehrstauglichkeit & 1324 \\
\hline 5.4 & Dokumentation & 1325 \\
\hline 5.4.1. & Allgemeines & 1325 \\
\hline 5.4.2. & Arbeitsunfähigkeit & 1325 \\
\hline 5.5 . & Benchmarking & 1325 \\
\hline
\end{tabular}




\begin{tabular}{|c|c|}
\hline $\begin{array}{l}\text { Fachgesellschaft/Arbeitsgemein- } \\
\text { schaft/Organisation }\end{array}$ & stimmberechtigte Autoren \\
\hline $\begin{array}{l}\text { Sektion Endoskopie im Auftrag der } \\
\text { Deutsche Gesellschaft für Verdau- } \\
\text { ungs- und Stoffwechselerkrankungen } \\
\text { e.V. (DGVS) }\end{array}$ & $\begin{array}{l}\text { Prof. Dr. Joachim Arnold } \\
\text { II. Medizinische Klinik, Diakoniekrankenhaus Rothenburg (Wümme) } \\
\text { Dr. Stefan von Delius } \\
\text { II. Medizinische Klinik und Poliklinik des Klinikusm rechts der Isar der Techni- } \\
\text { schen Universität München } \\
\text { PD Dr. Dirk Domagk } \\
\text { Medizinische Klinik und Poliklinik B Universitätsklinikum Münster } \\
\text { PD. Dr. Siegbert Faiss } \\
\text { III. Medizinische Abteilung Asklepios Klinik Barmbek, Hamburg } \\
\text { Dr. Dirk Hartmann } \\
\text { Medizinische Klinik C Klinikum Ludwigshafen } \\
\text { Dr. Christopher Hofmann } \\
\text { Klinik für Innere Medizin und Gastroenterologie, Katholisches Klinikum Mainz } \\
\text { Prof. Dr. Michael Jung } \\
\text { Klinik für Innere Medizin und Gastroenterologie, Katholisches Klinikum Mainz } \\
\text { PD Dr. Alexander Meining } \\
\text { II. Medizinische Klinik und Poliklinik des Klinikums rechts der Isar der Techni- } \\
\text { schen Universität München } \\
\text { Dr. Andrea Riphaus } \\
\text { Medizinische Klinik I, Klinikum Region Hannover GmbH, Krankenhaus Silaoh } \\
\text { Prof. Dr. Thomas Rösch } \\
\text { Klinik für Innere Medizin m. S. Hepatologie/Gastroenterologie Universitäts- } \\
\text { medizin Berlin Charité, Campus Virchow-Klinikum } \\
\text { PD Dr. Hans Seifert } \\
\text { Klinik für Innere Medizin I Klinikum Oldenburg GmbH } \\
\text { Prof. Dr. Andreas Sieg } \\
\text { Praxis für Gastroenterologie Heidelberg } \\
\text { Prof. Dr. Till Wehrmann } \\
\text { FB Gastroenterologie, Stiftung Deutsche Klinik für Diagnostik GmbH }\end{array}$ \\
\hline $\begin{array}{l}\text { Deutsche Gesellschaft für Anästhesie } \\
\text { und Intensivmedizin (DGAI) }\end{array}$ & $\begin{array}{l}\text { Prof. Dr. Wolfgang Heinrichs } \\
\text { Klinik für Anästhesiologie Universitätskliniken Mainz } \\
\text { PD Dr. Jörg Martin } \\
\text { Klinik für Anästhesiologie, Klinik am Eichert, Kliniken des Landkreises Göppin- } \\
\text { gen GmbH } \\
\text { Prof. Dr. Joachim Radke } \\
\text { Universitätsklinik für Anästhesiologie und operative Intensivmedizin, Univer- } \\
\text { sitätsklinikum der Martin-Luther- Universität Halle-Wittenberg }\end{array}$ \\
\hline $\begin{array}{l}\text { Berufsverband Niedergelassener } \\
\text { Gastroenterologen (bng) }\end{array}$ & $\begin{array}{l}\text { Dr. Marie-Luise Hermans } \\
\text { Praxis für Gastroenterologie Euskirchen } \\
\text { Dr. Bernd Wigginghaus } \\
\text { Praxis für Gastroenterologie Osnabrück }\end{array}$ \\
\hline $\begin{array}{l}\text { Chirurgische Arbeitsgemeinschaft für } \\
\text { Endoskopie und Sonographie (CAES) } \\
\text { der Deutschen Gesellschaft für Allge- } \\
\text { mein- und Viszeralchirurgie (DGAV) }\end{array}$ & $\begin{array}{l}\text { Dr. Martin Kraus } \\
\text { Klinik für Chirurgie, Universitätsklinikum Lübeck } \\
\text { PD Dr. Georg Kähler } \\
\text { Sektion Chirurgische Endoskopie, Universitätsklinik Mannheim }\end{array}$ \\
\hline $\begin{array}{l}\text { Gesellschaft für Recht und Politik im } \\
\text { Gesundheitswesen (GRPG) }\end{array}$ & $\begin{array}{l}\text { Dr. Horst Bitter } \\
\text { Prof. Dr. Dr. Alexander Friedrich Ehlers } \\
\text { Rechtsanwaltssocietät Ehlers, Ehlers \& Partner }\end{array}$ \\
\hline $\begin{array}{l}\text { Deutsche Gesellschaft für Endosko- } \\
\text { pieassistenzpersonal (DEGEA) }\end{array}$ & $\begin{array}{l}\text { Ulrike Beilenhoff } \\
\text { DEGEA, Ulm }\end{array}$ \\
\hline $\begin{array}{l}\text { Deutsche Morbus Crohn/Colitis ulce- } \\
\text { rosa Vereinigung e. V. (DCCV) }\end{array}$ & $\begin{array}{l}\text { Dr. Susanne In der Smitten } \\
\text { Deutsche Morbus Crohn/Colitis ulcerosa Vereinigung DCCV-Bundesgeschäfts- } \\
\text { stelle Leverkusen }\end{array}$ \\
\hline
\end{tabular}

Tab. 1 Leitliniengruppe: Beteiligte Fachgesellschaften und Organisationen.

\section{Einleitung}

$\nabla$

Hintergrund, Rationale und Ziele der Leitlinie

Der Stellenwert der Sedierung in der gastrointestinalen Endoskopie hat in den letzten Jahren zunehmend an Interesse gewonnen und ist derzeit Gegenstand vieler, teils auch kontroverser Diskussionen.

Ein Kernpunkt ist die differenzierte Indikationsstellung: Die Notwendigkeit einer Prämedikation bei gastroenterologischer Endoskopie ist nicht bei allen Eingriffen gegeben, sondern hängt vielmehr von der Art der Untersuchung, der Untersuchungsdauer, der Komplexität und der Invasivität der Untersuchung sowie von Patientenmerkmalen ab. Allerdings trägt die Prämedikation sowohl für den Patienten, als auch den Untersucher erheblich zum Untersuchungskomfort bei und schafft insbesondere bei komplexen therapeutischen Eingriffen oftmals erst die Voraussetzung für die erfolgreiche und risikoarme Untersuchung. Während in den USA und Großbritannien in bis zu $88 \%[1,2]$ der endoskopischen Untersuchungen eine Sedierung der Patienten erfolgt, lag die Sedierungsfrequenz in Deutschland und der 
Schweiz Mitte der 90er-Jahre mit ca. 9\% [3, 4] deutlich niedriger. Aktuellste Umfrageergebnisse einer „bundesweiten Evaluation zur Sedierung in der gastrointestinalen Endoskopie in Deutschland" zeigen jedoch auch bei uns eine deutliche Zunahme der Sedierungsfrequenz bei endoskopischen Eingriffen von bis zu $88 \%$ [5]. Dies ist am ehesten bedingt durch eine Zunahme an interventionellen Maßnahmen und zum anderen Folge des Patientenwunsches, z.B. im Rahmen der Kolonkarzinomvorsorge. Neben der zunächst lange Zeit gebräuchlichsten Form der Sedierung mittels Sedativa wie Benzodiazepinen, teils in Kombination mit Opoiden, findet zunehmend das kurzwirksame Hypnotikum Propofol Anwendung, mit einer Plasma-Halbwertszeit von 7-8 min. Der Vorteil bei der Anwendung von Propofol, im Vergleich zu Benzodiazepinen, liegt in einer signifikant kürzeren Aufwachzeit, bei gleich guter Patiententoleranz [6, 7] sowie einer deutlich rascheren Erholung der psychomotorischen Fähigkeiten unter Verwendung eines Fahrsimulators [8]. Allerdings können mit einer einzelnen Dosis Propofol mitunter verschiedene Sedierungstiefen durchschritten werden, sodass die Sedierung unter Umständen tiefer wird als beabsichtigt. Auch steht für Propofol anders als bei Midazolam kein Antagonist zur Verfügung. Trotz nicht eindeutiger Datenlage gilt derzeit in den Empfehlungen der verschiedenen Fachgesellschaften noch die 24-h-Regel, weder aktiv noch passiv am Straßenverkehr teilzunehmen [9-11].

Sehr aktuell wird derzeit die Propofol-Sedierung durch medizinisches Assistenzpersonal („NAPS“, nurse administered propofol Sedation) diskutiert. Zunehmender Kostendruck unter DRGBedingungen und die damit notwendige Kostenreduktion für die einzelne Untersuchung ist Ausgangspunkt einer Diskussion, ob geschultes nicht ärztliches Assistenzpersonal die Sedierung vornehmen kann. Derzeit wurden mehr als 200000 Patienten von sog. NAPS sediert, ohne dass die Notwendigkeit einer endotrachealen Intubation bestand [12-18]. Allerdings wurden hier meist gesunde Patienten im Rahmen diagnostischer Untersuchungen und der Verwendung von eher niedrigen PropofolDosierungen sediert. Eine unkritische Übernahme dieses Konzepts ist daher in keinem Fall zu empfehlen. Vielmehr bedarf es in Deutschland noch festzulegender Voraussetzungen, die dieses Vorgehen für den Patienten sicher machen (z. B. entsprechende Ausbildungskurse zur Thema Sedierung und Notfallmanagement).

Welche strukturellen Voraussetzungen für eine Sedierung in der gastrointestinalen Endoskopie und die Beherrschung möglicher sedierungsassoziierter Komplikationen erforderlich sind, soll durch die von verschiedenen medizinischen Fachgesellschaften (u. a. Gastroenterologen, Chirurgen und Anästhesisten), und Patientenselbsthilfeorganisationen, auch unter Berücksichtigung juristischer Aspekte, jetzt vorgelegte S3-Leitlinie „Sedierung in der gastrointestinalen Endoskopie“ festgelegt werden.

Die wesentliche Rationale für die Erstellung der Leitlinie ist somit der zunehmende Stellenwert der Sedierung in der gastrointestinalen Endoskopie. Neben der optimalen Patientenvorbereitung, die zusätzlich zu einer adäquaten Aufklärung über die Sedierung auch die Risikostratifizierung des einzelnen Patienten beinhaltet, soll ein Überblick über die derzeit gebräuchlichsten Sedativa und Analgetika (insbesondere unter Berücksichtigung der zunehmend Verwendung findenden kurzwirksamen Substanzen), deren Wirkungs- und Nebenwirkungsprofil gegeben werden.

Neben der medikamentösen Therapie mit unterschiedlichen Substanzen werden diese hinsichtlich ihrer Wirksamkeit und Effektivität bei der Untersuchung sowie ihres Risikoprofils (ins- besondere bei den einzelnen Risikogruppen) verglichen. Darüber hinaus sollen Patientenpräferenz und das Qualitätsmanagement der Sedierung unter entsprechendem Monitoring sowie das Zwischenfall-Management dargelegt werden.

Die Ziele der S3-Leitlinie „Sedierung in der gastrointestinalen Endoskopie" sind wie folgt definiert:

- Berücksichtigung aktueller Erkenntnisse der evidenzbasierten Medizin und anerkannte Verfahren der Sedierung und des Monitorings in der Umsetzung der Leitlinie.

- Vergleich der medikamentösen Therapie unterschiedlicher Substanzen hinsichtlich ihrer Wirksamkeit und Effektivität bei der Untersuchung sowie ihres Risikoprofils (insbesondere bei den einzelnen Risikogruppen).

- Darlegung von Patientenpräferenz und dem Qualitätsmanagement der Sedierung unter entsprechendem Monitoring sowie dem Zwischenfall-Management.

- Unterstützung von Ärzten und Patienten bei medizinischen Entscheidungen durch evidenzbasierte und formal konsentierte Empfehlungen.

- Unterstützung der Einbindung der Patientinnen und Patienten in Therapieentscheidungen unter Berücksichtigung ihrer individuellen Bedürfnisse.

- Flächendeckende Umsetzung einer multidisziplinären, qualitätsgesicherten und sektorübergreifenden Versorgung von Patienten, die im Rahmen einer Endoskopie einer Sedierung bedürfen.

- Unterstützung der Dokumentation von im Rahmen der Sedierung auftretenden Komplikationen.

- Systematische Berücksichtigung der Empfehlungen in der Aus-, Fort- und Weiterbildung und in Qualitätsmanagementsystemen.

- Schaffung einer Grundlage für inhaltlich gezielte ärztliche und pflegerische Aus-, Fort- und Weiterbildungsmaßnahmen.

- Systematische Berücksichtigung der Empfehlungen und daraus abgeleiteten Qualitätsindikatoren in der externen, vergleichenden Qualitätssicherung und Vereinheitlichung der Dokumentationsanforderungen.

- Flächendeckende Umsetzung einer qualitätsgesicherten Versorgung der Patienten während einer Sedierung im Rahmen der gastrointestinalen Endoskopie.

- Optimierung der Patientensicherheit.

In Ergänzung zu und durch Vernetzung mit den bereits bestehenden Empfehlungen zur Sedierung in der gastrointestinalen Endoskopie durch Nicht-Anästhesisten [9-11, 19-25] sowie den weiteren, oben genannten Projekten und Maßnahmen soll mittel- und langfristig die Patientensicherheit erhöht werden.

Organisatorischer Ablauf methodischer Grundlagen des Konsensusprozesses (Kurzform des Leitlinienreports) Nach Beauftragung durch die DGVS wurde der organisatorische Ablauf mit der stellvertretenden Vorsitzenden der AWMF, Frau PD Dr. med. Ina Kopp besprochen, die auch die weitere methodische Betreuung der Leitlinie übernahm. Am 10.1.2007 erfolgte die Anmeldung des Leitlinienprojekts bei der AWMF (AWMFRegister-Nr. 014/021).

\section{Leitliniensteuergruppe und ihre Aufgaben}

Durch die Koordinatoren der federführenden Fachgesellschaft wurden die Zusammensetzung und Aufgabenbereiche der Leitliniensteuergruppe wie folgt festgelegt:

Leitlinienkoordination: Dr. Andrea Riphaus, Hannover Prof. Dr. Till Wehrmann, Hannover 
Projektmanagement:

Dr. Andrea Riphaus, Hannover

Prof. Dr. Till Wehrmann, Hannover

Birgit Weber, Hannover

Methodische Begleitung: PD Dr. Ina Kopp, AWMF, Marburg

Zu den Aufgaben der Steuergruppe gehörten die Kontaktaufnahme und Rückkopplung zu den beteiligten Fachgesellschaften und Organisationen, Umsetzung der methodischen Vorgaben für Leitlinien der Stufe 3 anhand eines Projektplans, Verwaltung der finanziellen Ressourcen, Unterstützung der inhaltlichen Arbeit der Experten, Zusammenführung und redaktionelle Bearbeitung der von den Experten in Arbeitsgruppen vorbereiteten Textentwürfe, Erstellung des Leitlinien-Methodenreports.

\section{Auswahlkriterien des Expertenkreises, Arbeitsgruppen und} ihre Aufgaben

Die Leitliniengruppe wurde von den Koordinatoren einberufen. Dabei wurden alle die Thematik der S3-Leitlinie unmittelbar betreffenden Fachgesellschaften, Arbeitsgemeinschaften und Organisationen angesprochen. Diese wurden gebeten, Experten als Mandatsträger zu ihrer Vertretung in den Abstimmungsprozessen (Konsensusverfahren) sowie für die inhaltliche Arbeit in themenspezifischen Gruppen zu benennen (zur Besetzung $\bullet$ Tab. 1, 2). Alle Experten wurden nach dem Prinzip der Fach- und Sachkompetenz ausgewählt und eingeladen. Ziel war die Gewährleistung einer dem Inhalt und dem Anwendungsbereich der Leitlinie entsprechenden Multidisziplinarität und Multiprofessionalität innerhalb der Leitliniengruppe. Eine Vertreterin der Selbsthilfeorganisationen wurde von Beginn an aktiv in den Leitlinienerstellungsprozess integriert mit dem Ziel, die Perspektive der Betroffenen stärker zu kontrastieren.

\section{Systematische Evidenzrecherche}

Die Literaturrecherche wurde dezentral innerhalb der Arbeitsgruppen mit Unterstützung durch das Leitlinienkoordinationsbüro durchgeführt. Die Suchstrategie für die Erstellung der Leitlinie wurde wie folgt festgelegt:

- Datenbanken: Pubmed, Cochrane Library

- Suchbegriffe:

- Allgemein: „sedation or conscious sedation“ und „endoscopy or gastrointestinal endoscopy“

Arbeitsgruppenspezifisch ( Tab.3)

- Zeitraum: 1990-2007

4079 Publikationen wurden so identifiziert. Nach einer Vorsichtung der Abstrakts durch die Arbeitsgruppenleiter, Ausschluss von nicht in englischer oder deutscher Sprache erhältlichen und inhaltlich nicht relevanten Arbeiten, wurden insgesamt 210 Quellen zur Bewertung ausgewählt. Zusätzlich erfolgte eine Handsuche und Ergänzung relevanter Arbeiten durch die Mitglieder der Arbeitsgruppen. So wurden zusätzlich 23 Publikationen in die die Leitlinie mit aufgenommen. Alle diese Quellen wurden vom Leitlinienkoordinationsbüro in eine Literaturdatenbank aufgenommen. 44 Arbeiten, die für mehr als eine Fragestellung relevant waren, wurden jeweils in Bezug auf diese Fragestellung gesondert bewertet und sind dementsprechend zum Teil mehrfach in den Evidenztabellen aufgeführt.

Zusätzlich zur Literaturrecherche erfolgte eine gezielte Leitlinien-Recherche nach folgender Strategie:

- Datenbank: Pubmed and Guidelines International Network (GIN), www.g-i-n.net Suchbegriff: sedation AND gastrointestinal endoscopy

- Zeitraum: 1990-2007
Hierdurch wurden 11 Publikationen identifiziert.

Die Bewertung der relevanten Literatur erfolgte durch die Mitglieder der einzelnen Arbeitsgruppen ( Tab. 2, 3).

\section{Klassifikation der Evidenzgrade, der Empfehlungsgrade} und der Konsensusstärke

Die vorliegende Leitlinie nutzt als Grundlage zur Evidenzdarlegung die Evidenzkategorien des Oxford Centre for Evidence Based Medicine (s. Anhang I). Die Graduierung der Empfehlungen erfolgte unter Bezugnahme auf den aktuellen Methoden-Report des Programms für Nationale Versorgungsleitlinien (s. Anhang II).

Tab. 2 Kapitelstruktur der Arbeitsgruppen der S3-Leitlinie 2008.

\begin{tabular}{|c|c|}
\hline Kapitel/Themenkomplex & Arbeitsgruppe, Sprecher \\
\hline \multicolumn{2}{|c|}{ Arbeitsgruppe I: Indikationen/Ziele/bekannte Risiken/Patienten/Qualitätsziele } \\
\hline - Ziele & $\begin{array}{l}\text { Jung, Seifert, Domagk, In der } \\
\text { Smitten }\end{array}$ \\
\hline \multicolumn{2}{|l|}{ - bekannte Risiken } \\
\hline \multicolumn{2}{|l|}{ - Patientenaspekte } \\
\hline \multicolumn{2}{|l|}{ - Qualitätsziele } \\
\hline \multicolumn{2}{|l|}{ Arbeitsgruppe Il: Pharmakologie } \\
\hline - Sedativa & $\begin{array}{l}\text { Meining, Heinrichs, von Delius, } \\
\text { Martin, Radke }\end{array}$ \\
\hline \multicolumn{2}{|l|}{ - Analgetika } \\
\hline \multicolumn{2}{|l|}{ - vegetativ wirksame Pharmaka } \\
\hline \multicolumn{2}{|l|}{ - Kombinationstherapien } \\
\hline \multicolumn{2}{|l|}{ - Applikationstechniken } \\
\hline \multicolumn{2}{|l|}{ Arbeitsgruppe III: Strukturqualität } \\
\hline - persönliche Voraussetzungen & $\begin{array}{l}\text { Arnold, Hartmann, Kraus, Radke, } \\
\text { Wehrmann }\end{array}$ \\
\hline \multicolumn{2}{|l|}{ - personelle Voraussetzungen } \\
\hline \multicolumn{2}{|l|}{ - apparative Ausstattung } \\
\hline \multicolumn{2}{|l|}{ Arbeitsgruppe IV: Prozessqualität } \\
\hline - Aufklärung, Einwilligung & $\begin{array}{l}\text { Sieg, Heinrichs, Beilenhoff, Ehlers, } \\
\text { Hofmann, Kähler, In der Smitten }\end{array}$ \\
\hline \multicolumn{2}{|l|}{ - Durchführung der Sedierung } \\
\hline \multicolumn{2}{|l|}{$\begin{array}{l}\text { - Monitoring, klinische Überwa- } \\
\text { chung, Steuerung }\end{array}$} \\
\hline \multicolumn{2}{|l|}{$\begin{array}{l}\text { - Zwischenfall Management, } \\
\text { Sicherung vitaler Funktionen }\end{array}$} \\
\hline \multicolumn{2}{|l|}{ Arbeitsgruppe V: Ergebnissqualität } \\
\hline - Entlassungskriterien & $\begin{array}{l}\text { Riphaus, Hermans, Bitter, Faiss, } \\
\text { Wigginghaus, Rösch }\end{array}$ \\
\hline - Street-Fitness/Arbeitsfähigkeit & \\
\hline $\begin{array}{l}\text { - Dokumentation, externe Quali- } \\
\text { tätssicherung, „Benchmarking“ }\end{array}$ & \\
\hline
\end{tabular}

Tab. 3 Literaturrecherche.

\begin{tabular}{|lcc|}
\hline $\begin{array}{l}\text { Themenkomplex } \\
\text { Themenkomplex I }\end{array}$ & $\begin{array}{l}\text { Referenzen } \\
\text { initial }\end{array}$ & $\begin{array}{c}\text { Für die LL als relevant } \\
\text { bewertete Referenzen }\end{array}$ \\
\hline $\begin{array}{l}\text { Indikationen } \\
\text { Themenkomplex II } \\
\text { Phamakologie }\end{array}$ & $\begin{array}{l}3179 \\
\text { (ohne narrati- } \\
\text { ve Reviews) }\end{array}$ & 72 \\
\hline $\begin{array}{l}\text { Themenkomplex III } \\
\text { Strukturqualität }\end{array}$ & 142 & 28 \\
\hline $\begin{array}{l}\text { Themenkoplex IV } \\
\text { Prozessqualität }\end{array}$ & 335 & 86 \\
\hline $\begin{array}{l}\text { Themenkomplex V } \\
\text { Ergebnissqualität }\end{array}$ & 355 & 16 \\
\hline
\end{tabular}




\begin{tabular}{|c|c|c|c|c|}
\hline \multirow{2}{*}{$\begin{array}{l}\text { Evidenzgrad } \\
\text { CEBM }\end{array}$} & \multicolumn{2}{|c|}{ vereinfachte Definition der Quellen } & \multirow{2}{*}{$\begin{array}{l}\text { Empfehlungs- } \\
\text { grad }\end{array}$} & \multirow[t]{2}{*}{ Beschreibung } \\
\hline & Therapie & Diagnostik & & \\
\hline I & $\begin{array}{l}\text { randomisierte kontrol- } \\
\text { lierte Studien }\end{array}$ & $\begin{array}{l}\text { validierende Kohorten- } \\
\text { studien }\end{array}$ & A & starke Empfehlung \\
\hline II & $\begin{array}{l}\text { kontrollierte Studien } \\
\text { ohne Randomisierung }\end{array}$ & $\begin{array}{l}\text { explorative Kohorten- } \\
\text { studien }\end{array}$ & B & Empfehlung \\
\hline III-V & Beobachtungs-Studien, & ertenmeinung & 0 & Empfehlung offen \\
\hline
\end{tabular}

Tab. 4 Vereinfachtes Schema zur Überleitung von Evidenz- und Empfehlungsstärken.
Tab.5 Definition der Konsensstärke.

\begin{tabular}{|ll|}
\hline starker Konsens & $\geq 98 \%$ der Teilnehmer \\
\hline Konsens & $>75-98 \%$ \\
\hline mehrheitliche Zustimmung & $>50-75 \%$ \\
\hline kein Konsens & $\leq 50 \%$ der Teilnehmer \\
\hline
\end{tabular}

Die in der Leitlinie verwendeten Bezeichnungen und das Schema zur Überleitung von Evidenz- zu Empfehlungsgraden sind in Tab. 4 zusammenfassend und vereinfacht dargestellt.

Für die Durchführung der formalen Konsensusverfahren wurden die Techniken des Nominalen Gruppenprozesses (NGP), formale Konsensuskonferenzen und die Delphi-Technik eingesetzt. Die Abstimmungsverläufe mit allen inhaltlichen Beiträgen sowie die Abstimmungsergebnisse und die Bewertung der Stärke des Konsens wurden unter Darstellung der Gründe dokumentiert ( Tab.5).

\section{Konsensusverfahren}

Im Rahmen eines ersten Konsensusprozesses im Januar 2007 in Hannover wurde das methodische Konzept für die LL-Erstellung sowie die Struktur und die Schlüsselfragen für die Literaturrecherche festgelegt. Nachfolgend wurden die Arbeitsgruppen festgelegt ( Tab.2). Weiterhin wurde die Vollständigkeit der LL-Gruppe überprüft. Eine Nachbenennung weiterer Experten oder die Hinzuziehung weiterer Fachgruppen wurde von der LL-Gruppe nicht als erforderlich angesehen.

Im Rahmen eines zweiten Konsensusprozesses erfolgte dann durch die Mitglieder der Arbeitsgruppen die Auswahl von Quellen, die Formulierung der Kernaussagen und Empfehlungen, die Darstellung der zugrunde liegenden Evidenzstärke und des sich aus der Primärliteratur ergebenden Empfehlungsgrads. Im Rahmen des zweiten, formalisierten Konsensusverfahrens wurden die so vorgeschlagenen Kernaussagen und Empfehlungen einschließlich der Graduierung diskutiert und unter Vorgabe der Kriterien (Konsensusaspekte) für die Graduierung der Empfehlungen abgestimmt. Abweichungen des Evidenzgrads vom Empfehlungsgrad wurden entsprechend begründet. In der Leitlinie werden alle Kernaussagen und Empfehlungen hinsichtlich der Evidenzstärke und die Empfehlungen zusätzlich dem Grad der Empfehlung ausgewiesen. Die Konsensfindung erfolgte im Rahmen einer 2- und einer 1-tägigen strukturierten Konsensuskonferenz der gesamten Leitliniengruppe im Juni 2007 und im September 2007 in Hannover bzw. Frankfurt am Main.

Der Ablauf der Konsensuskonferenzen erfolgte in mehreren Schritten:

Teil 1: Kurzvorträge

- Einführung in die Technik des formalen Konsensusverfahrens durch die Moderatorin.

- Darstellung des medizinisch-wissenschaftlichen Kenntnisstandes für jeden Themenkomplex durch die Arbeitsgruppensprecher.
- Gelegenheit zu Rückfragen zum methodischen Vorgehen, zur Begründung der Ergebnisse im Plenum.

Teil 2: Strukturierte Konsensfindung

- Kapitelweises Vorgehen, Aufruf jeder Kernaussage bzw. jeder Empfehlung einzeln durch die Moderatorin.

- Registrierung von Stellungnahmen aus dem Plenum durch die Moderatorin.

- Klarstellung und Begründung alternativer Vorschläge.

- Vorabstimmung über Erstentwurf und alle Alternativen.

- Feststellung von Diskussionspunkten und Dissens.

- Debattieren und Diskutieren.

- Endgültige Abstimmung.

Für die im Rahmen dieser Treffen nicht zur Abstimmung gekommenen Kernaussagen der AG II Pharmakologie und der AG IV Prozessqualität wurde eine Abstimmung im modifizierten Delphiverfahren durchgeführt.

Adressaten waren alle Mitglieder der Leitliniengruppe. Dabei wurden zu jedem Kapitel folgende Angaben erfragt:

- Zustimmung

- Keine Zustimmung

- Konkrete und begründete Änderungsvorschläge mit Literaturangabe

Im Rahmen des Delphi-Verfahrens wurden alle Mitglieder der Leitliniengruppe zusätzlich gebeten, aus ihrer Sicht relevante Qualitätsziele zu benennen, die sich aus Empfehlungen mit hohem Evidenzgrad (1-2) ableiten lassen.

Die Ergebnisse dieser ersten Befragung (erste Delphirunde) wurden zusammengefasst, der Leitliniengruppe zurückgespiegelt und die nicht konsensfähigen Abschnitte mit Änderungsvorschlägen im Juli 2008 erneut zur Abstimmung gebracht (zweite Delphirunde).

Die abschließende Konsentierung des Gesamtleitlinientexts erfolgte ebenfalls schriftlich, im modifizierten Delphiverfahren im August 2008.

Abschließend wurde der Gesamtleitlinientext im September 2008 den Präsidien und Vorständen der beteiligten Fachgesellschaften und Organisationen zur Zustimmung vorgelegt.

\section{Verbreitung und Implementierung der Leitlinie}

Die S3-Leitlinie „Sedierung in der gastrointestinalen Endoskopie" wird in folgenden Formaten publiziert:

1. Kurzversion in deutscher und englischer Sprache (Zeitschriften mit Peer-review-Verfahren), Kurzversion der Patienten-/ Patientinneninformation.

2. Vollversion (Kurz-, Lang-, Patientinnenversion und Methodenreport) im Internet (www.awmf-leitlinien.de; www. dgvs.de, www.dgai.de, www.dgvc.de, www.bng.de, www. dccv.de, www.degea.de).

3. Vollversion in englischer Sprache im Internet (Guidelines International Network, GIN: www.g-i-n.net). 
Die Publikation der S3-Leitlinie wird in elektronischer und gedruckter Form erfolgen. Über die Internet-Portale der AWMF (www.awmf-leitlinien.de, AWMF-Register Nummer 021/014), der Deutschen Gesellschaft für Verdauungs- und Stoffwechselerkrankungen (www.dgvs.de, Rubrik Leitlinien) und der Deutschen Gesellschaft für Anästhesie und Intensivmedizin für (www.dgai.de) wird die Leitlinie mit folgenden Elementen frei zugänglich gemacht:

- Langfassung: Volltext, Empfehlungen und Algorithmen mit ausführlichen Hintergrundinformationen und kurzgefasstem Methodenreport zur Begründung der einzelnen Empfehlungen und umfassendem Literaturverzeichnis.

- Kurzfassung im Anhang der Langfassung: Zusammenfassung der Versorgungsempfehlungen mit Angabe der Evidenzstärken und Empfehlungsgrade.

- Leitlinien-Methodenreport: ausführliche Darlegung der Methodik des Entwicklungsprozesses.

Sonderformate sind Bestandteile der Implementierungsstrategie. Es wird explizit angeregt, unter Bezugnahme auf die unter 1-4 ausgewiesenen Publikationen die Leitlinie in die Anwendung zu überführen. Hierzu zählen z.B.:

- Umsetzung von Algorithmen in Klinik und Praxis (klinische lokale Behandlungspfade, Schulungen und entsprechende lokale Entwicklung von Hilfsmitteln: z. B. Kitteltaschenformate und Einbindung in unterstützende Medien: elektronische Einbindungen in Klinik- und Praxis-Informationssysteme im Rahmen von Qualitätsmanagement).

- Einbindung der Leitlinie in existierende und zukünftige Schulungsprogramme (z.B. Simulationswokshops Analgosedierung und Komplikationsmanagement).

- Umsetzung der Patienteninformation in der Öffentlichkeitsarbeit, z. B. Postkartenformate, Internetdarstellungen, Broschüren.

Die Verbreitung und Implementierung der Leitlinie wird weiterhin durch die Steuergruppe unterstützt durch:

- öffentlichkeitswirksame Darstellung durch die Träger der Leitlinienerstellung,

> Pressemeldung an den Informationsdienst Wissenschaft idw (idw-online.de),

- Pressekonferenzen,

direkte Ansprache der Adressaten der Leitlinie,

- Beiträge in Fachzeitschriften, Buchbeiträge,

- Kongresse, Vorträge, Seminare,

- Unterstützung der Erstellung von Materialien für die Fortund Weiterbildung (CME-Akkreditierung entsprechend den Landesärztekammern).

\section{Finanzierung der Leitlinie und Darlegung möglicher} Interessenskonflikte

Die Erstellung der S3-Leitlinie wurde durch die Deutsche Gesellschaft für Verdauungs- und Stoffwechselkrankheiten mit $30000 €$ gefördert. Diese Mittel wurden eingesetzt für Personalkosten (Leitliniensekretariat), im Rahmen der Planungstreffen und Konsensusverfahren angefallene Kosten (Reisekosten für alle Teilnehmer, Saalmieten, Technik und Catering), Kosten für Büromaterial und Aufwandsentschädigungen für den Einsatz externer Experten zur methodischen Unterstützung. Die Erstellung der Leitlinie erfolgte in redaktioneller Unabhängigkeit von der finanzierenden Organisation. Den Autoren und Teilnehmern der Konsensusverfahren ist zu danken für ihre ausschließlich ehrenamtliche Arbeit. Alle Mitglieder der Leitliniengruppe legten eine schriftliche Erklärung zu eventuell be- stehenden Interessenskonflikten, vor allem gegenüber der Industrie vor.

\section{Gültigkeitsdauer und Aktualisierungsverfahren}

Die Leitlinie ist bis spätestens Dezember 2012 gültig. Eine komplette Revision und Neuauflage wird zu diesem Zeitpunkt angestrebt. Zwischenzeitliche Erkenntnisse, die eine Aktualisierung einzelner Abschnitte oder Empfehlungen erforderlich machen können, werden von der Leitliniengruppe beobachtet. Entsprechende Hinweise an den Koordinator sind auch von den Adressaten der Leitlinie erwünscht. Es wird angestrebt, dadurch kontinuierlich bedarfsgerechte Aktualisierungen vorzunehmen.

Das Datum der Veröffentlichung, das Datum der nächsten geplanten Überarbeitung sowie die Anmeldung der geplanten und/ oder zwischenzeitlichen Aktualisierungen werden im öffentlich zugänglichen Verzeichnis der AWMF (http://www-awmf-leitlinien.de) ausgewiesen. Gültig ist nur die jeweils neueste Version gemäß dem AWMF-Register.

\section{Anhang I \\ $\nabla$}

Methodische Qualität der wissenschaftlichen Belege: Klassifizierung der Evidenzstärke.

\begin{tabular}{|c|c|}
\hline Level & Studien zu Therapie/Prävention/Ätiologie \\
\hline $1 \mathrm{a}$ & $\begin{array}{l}\text { systematische Übersicht über randomisierte kontrollierte } \\
\text { Studien (RCT) }\end{array}$ \\
\hline $1 b$ & eine RCT (mit engem Konfindenzintervall) \\
\hline $1 c$ & Alle-oder-keiner-Prinzip \\
\hline $2 \mathrm{a}$ & systematische Übersicht gut geplanter Kohortenstudien \\
\hline $2 b$ & $\begin{array}{l}\text { eine gut geplante Kohortenstudie oder eine RCT minderer } \\
\text { Qualität }\end{array}$ \\
\hline $2 c$ & Outcome-Studien, ökologische Studien \\
\hline $3 a$ & systematische Übersicht über Fallkontrollstudien \\
\hline $3 b$ & eine Fallkontrollstudie \\
\hline 4 & $\begin{array}{l}\text { Fallserien oder Kohorten-/Fallkontrollstudien minderer } \\
\text { Qualität }\end{array}$ \\
\hline 5 & $\begin{array}{l}\text { Expertenmeinung ohne explizite Bewertung der Evidenz oder } \\
\text { basierend auf physiologischen Modellen/Laborforschung }\end{array}$ \\
\hline Level & Studien zu Diagnose \\
\hline 1a & $\begin{array}{l}\text { systematische Übersicht über Level-1-diagnostische Studien } \\
\text { oder diagnostische Entscheidungsregel, begründet auf } \\
\text { 1b-Studien, validiert in verschiedenen klinischen Zentren }\end{array}$ \\
\hline $1 b$ & $\begin{array}{l}\text { Validierungs-Kohortenstudie mit gutem Referenzstandard } \\
\text { oder diagnostische Entscheidungsregel, validiert in einem } \\
\text { Zentrum }\end{array}$ \\
\hline $1 c$ & Alle-oder-keiner-Prinzip \\
\hline $2 \mathrm{a}$ & systematische Übersicht über Level-2-diagnostische Studien \\
\hline $2 b$ & explorative Kohortenstudie mit gutem Referenzstandard \\
\hline $3 a$ & systematische Übersicht über Level-3-diagnostische Studien \\
\hline $3 b$ & $\begin{array}{l}\text { nicht konsekutive Studie oder ohne Konsistenz der angewen- } \\
\text { deten Referenzstandards }\end{array}$ \\
\hline 4 & $\begin{array}{l}\text { Fallkontrollstudie, schlechte oder nicht unabhängige Refe- } \\
\text { renzstandards }\end{array}$ \\
\hline 5 & $\begin{array}{l}\text { Expertenmeinung ohne explizite Bewertung der Evidenz oder } \\
\text { basierend auf physiologischen Modellen/Laborforschung }\end{array}$ \\
\hline
\end{tabular}

Level of Evidence/LoE nach Oxford Centre for Evidence Based Medicine 2001

B. Philipps, D. Sackett, D. Badenoch, S. Strauss, B. Haynes, M. Dawes (Centre of Evidence Based Medicine Oxford 2001, http://cebm.jr2ox.ac.uk/docs/levels.html) 
Von der Evidenz zur Empfehlung:

Visualisierung der klinischen Beurteilung

als Prozess der Kriteriengestützten Konsensusentscheidung

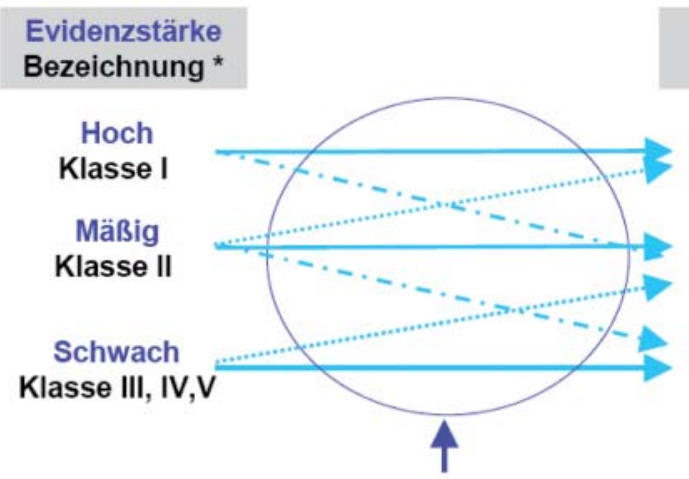

Empfehlungsgrad Symbole **

Starke Empfehlung A, $\Uparrow \Uparrow$

Empfehlung

$\mathbf{B}, \Uparrow$

Empfehlung offen

$0, \Leftrightarrow$
Abb. $1 *$ * Nach Oxford Centre of Evidence based Medicine, ${ }^{* *}=$ Symbole der Empfehlungsgraduierung im Programm für Nationale Versorgungsleitlinien. Die Empfehlungen werden nach Möglichkeit analog formuliert: Starke Empfehlung: „soll“ Empfehlung: „sollte“ Empfehlung offen: „kann“ (Handlungsoption). Negativ-Empfehlungen werden sprachlich ausgedrückt („,nicht“) bei gleichen Symbolen.

Kriterien für die Graduierung (Konsensusaspekte):

- Konsistenz der Studienergebnisse

- Klinische Relevanz der Endpunkte und Effektstärken

- Nutzen-Risiko-Verhältnis

- Ethische Verpflichtungen

- Patientenpräferenzen

- Anwendbarkeit, Umsetzbarkeit

\section{Anhang II}

$\nabla$

Von der Evidenz zur Empfehlung: Klassifizierung der Empfehlungsstärke

Graduierung von Empfehlungen nach: Bundesärztekammer, AWMF, Kassenärztliche Bundesvereinigung (Hrsg). Programm für Nationale Versorgungsleitlinien - Methodenreport. Berlin, ÄZQ www.methodik.n-v-l.de. Abbildung: Kopp I, Lelgemann M, Ollenschläeger G: EbM und Leitlinien. In: Kunz $\mathrm{R}$ et al. (Hrsg): Lehrbuch Evidenzbasierte Medizin in Klinik und Praxis. 2. Aufl. 2007, Deutscher Ärzteverlag, Köln ( Abb.1).

\section{Indikationen/Ziele/bekannte Risiken/Patienten/ Qualitätsziele}

\subsection{Empfehlung Sedierungsangebot}

Empfehlung

Jedem Patienten soll eine Sedierung vor der Endoskopie angeboten werden. Dabei soll über Vor- und Nachteile einer Sedierung diskutiert werden.

Empfehlungsgrad: A, Evidenzstärke 5, Konsens.

\section{Anmerkungen}

Jeder Patient hat das Recht auf eine möglichst schmerz- und stressfreie Endoskopie. Daher erscheint es ethisch nicht vertretbar, grundsätzlich Patienten eine Sedierung vorzuenthalten [26]. Endoskopische Untersuchungen können unangenehm sein, sodass eine Sedierung gewünscht oder zu empfehlen ist. Insbesondere länger dauernde, endoskopisch-interventionelle Eingriffe diffiziler Art (z.B. ERCP, schwierige Resektions- oder DrainageVerfahren) setzen zudem die Vermeidung unwillkürlicher Bewegungen des Patienten voraus. Darum soll eine Sedierung prinzipiell jedem Patienten angeboten werden. Nach entsprechender Aufklärung über die Sedierung soll den diesbezüglichen Patientenwünschen möglichst Rechnung getragen werden.
Empfehlung

Grundsätzlich können einfache endoskopische Untersuchungen (Gastroskopie, Sigmoidoskopie, Koloskopie etc.) bei geeigneten Patienten auch ohne Sedierung durchgeführt werden.

Empfehlungsgrad: A, Evidenzstärke 2b, starker Konsens.

\section{Anmerkungen}

Eine randomisierte kontrollierte Studie [27] und zwei prospektive Kohorten-Studien $[28,29]$ belegen diese Aussage, wobei die Zustimmung zu einer unsedierten Koloskopie nur 88\% betrug $[28,29]$. In einer weiteren Studie stimmten nur 20\% der Befragten einer Koloskopie ohne Sedierung zu. Männliches Geschlecht, höhere Bildung und geringere Ängstlichkeit waren positive prädikative Faktoren für den Patientenwunsch nach einer Koloskopie ohne Sedierung [30].

\subsection{Empfehlung zur Indikationsstellung für eine Sedie- rung}

Empfehlung

Bei der Entscheidung für oder gegen ein Verfahren zur Sedierung und/oder Analgesie sollen folgende Aspekte berücksichtigt werden:

- Patientencharakteristika(Risikoprofil,Komorbidität,Präferenzen)

- Art des endoskopischen Eingriffs (Anlass, Dauer, Invasivität)

- Strukturelle Voraussetzungen

Empfehlungsgrad: A, Evidenzstärke 5, starker Konsens.

\subsection{Empfehlung Untersuchungsqualität}

Empfehlung

Eine Aussage zur Beeinflussung der Komplikationsrate (durch den endoskopischen Eingriff) bei Verzicht auf eine Sedierung ist nicht möglich.

Evidenzstärke 5, starker Konsens.

\section{Anmerkungen}

Die Notwendigkeit einer Prämedikation bei gastroenterologischen Eingriffen ist zweifelsfrei nicht bei allen endoskopischen 
Eingriffen obligat und letztlich abhängig von der Art der Untersuchung, der Untersuchungsdauer, Komplexität und Invasivität der Untersuchung. Die Prämedikation trägt sowohl für den Patienten als auch für den Untersucher erheblich zum Untersuchungskomfort bei und schafft insbesondere bei komplexen therapeutischen Eingriffen oftmals erst die Voraussetzung für die Durchführung einer erfolgreichen und risikoarmen Untersuchung. Auch spielt die Patientenpräferenz eine nicht zu unterschätzende Rolle. Vorerfahrung mit bereits „durchgemachten“ Untersuchungen, bestehende Ängstlichkeit und die Einsichtsfähigkeit des Patienten sollten hierbei berücksichtigt werden. Darüber hinaus gibt es oftmals kulturell bedingte weltweit große Unterschiede im Umgang mit der Sedierung bei der gastro-intestinalen Endoskopie. Beispielsweise erfolgt in den USA und Großbritannien in bis zu 88\% [1, 2] der endoskopischen Untersuchungen eine Sedierung der Patienten. Hingegen lag die Sedierungsfrequenz in Deutschland und der Schweiz in den 90erJahren mit ca. 9\% [3, 4] deutlich niedriger, nimmt aber mit der Komplexität der Untersuchung zu. Aktuellste Umfrageergebnisse zeigen jedoch auch in Deutschland eine deutliche Zunahme der Sedierungsfrequenz bei endoskopischen Eingriffen von bis zu $87 \%$ [5]. Dies ist vermutlich zum einen bedingt durch eine vermehrte Häufung interventioneller Untersuchungen und zum anderen als Folge des Patientenwunsches z.B. im Rahmen der Kolonkarzinomvorsorge.

Allerdings existieren kaum Studien, die die Sicherheit der diagnostischen wie auch therapeutischen Endoskopie mit oder ohne Sedierung gegeneinander werten (siehe auch Evidenztabelle 1 und 2 im Methodenreport). Eine amerikanische Untersuchung verfehlte wegen mangelnder Patientenakzeptanz (hoher Sedierungswunsch) das angestrebte Vergleichsziel [27].

Das Ergebnis einer deutschen Studie zeigte, dass die Koloskopie in über $90 \%$ der Fälle ohne Sedierungsmaßnahme mit einer geringen Komplikationsrate vorgenommen werden kann [28], wobei allgemein bei den Patienten jedoch der Wunsch nach einer Prämedikation überwiegt $[28,31,32]$. Diese scheint das Ri- siko für Komplikationen durch die Endoskopie nicht nachhaltig zu beeinflussen.

So konnte in einer prospektiven Studie von Dillon et al. [33] bei 136 Kindern zur Koloskopie unter Allgemeinanästhesie gezeigt werden, dass die Perforationsrate nicht höher als bei Erwachsenen unter Sedierung war. Die Behauptung, es käme bei gut erhaltenen Schmerzreizen zu weniger Koloskopie-Perforationen, ist somit nicht belegt.

\subsection{Empfehlung Risikoabschätzung und Strukturqualität 1.4.1. Allgemeines}

Mit sedierend und analgetisch wirkenden Medikamenten können ineinander übergehende, nicht immer sicher abgrenzbare Zustände induziert werden, die von einer minimalen Sedierung (Anxiolyse) bis hin zur Allgemeinanästhesie reichen.

Sedierungs- und/oder Analgesieverfahren durch Nicht-Anästhesisten dürfen planbar keinen Sedierungsgrad erreichen, bei dem lebenserhaltende Reflexe beeinträchtigt oder ausgeschaltet werden.

Durchführung von geplanten Allgemeinanästhesien (mit Verlust des Bewusstseins/der Schutzreflexe) sind ausschließlich dem Anästhesisten vorbehalten.

Sollte im Einzellfall ein Sedierungsgrad erreicht werden, bei dem lebenserhaltende Reflexe beeinträchtigt oder ausgeschaltet werden (Allgemeinanästhesie) und der Eingriff fortgesetzt werden soll, ist ein Anästhesist hinzuzuziehen.

Trotz der fließenden, nicht immer sicher steuerbaren Übergänge lassen sich verschiedene Ausprägungsgrade der (Analgo-)Sedierung unterscheiden.

Der Grad (die Tiefe) der Sedierung kann durch eine validierte Skala bestimmt und eingeteilt werden. Während in der Anästhesie die modifizierte Richmond Agitation-Sedation-ScoreSkala (RASS-Skala) [34] ( Tab.6) gebräuchlich ist, wird in der Gastroenterologie meist die Einteilung der verschiedenen Sedierungsstadien nach der American Society of Anesthesiologists [19] ( Tab. 7) verwandt.

Tab. 6 Modifizierter Richmond-Agitation-Sedation-Score [34].

\begin{tabular}{|c|c|c|}
\hline Grad & Bezeichnung & Beschreibung \\
\hline 0 & aufmerksam und ruhig & \\
\hline-1 & schläfrig & nicht völlig alert, aber mindestens 10 -sekündige Wachphasen (Augen öffnen, Augenkontakt) bei Ansprache \\
\hline-2 & leichte Sedierung & Wachphasen (Augen öffnen, Augenkontakt) von weniger als 10 Sekunden Dauer bei Ansprache \\
\hline-3 & mittlere Sedierung & Bewegung oder Augen öffnen bei Ansprache (aber kein Augenkontakt) \\
\hline-4 & tiefe Sedierung & $\begin{array}{l}\text { keine Reaktion auf Ansprache, aber Bewegung oder Augen öffnen durch körperliche Stimulation (Schulter schüt- } \\
\text { teln oder Sternum reiben) }\end{array}$ \\
\hline-5 & nicht erweckbar & keine Reaktion auf Ansprache oder körperliche Stimulation \\
\hline
\end{tabular}

Tab. 7 Stadien der Sedierung (modifiziert nach der American Society of Anesthesiologists [19]).

\begin{tabular}{lllll} 
& $\begin{array}{l}\text { minimal } \\
\text { (Anxiolyse) }\end{array}$ & moderat & tief & Narkose \\
\hline $\begin{array}{l}\text { Reaktion auf } \\
\text { Ansprache }\end{array}$ & $\begin{array}{l}\text { Pat. reagiert } \\
\text { adäquat } \\
\text { aufverbale } \\
\text { Kommandos }\end{array}$ & $\begin{array}{l}\text { Bewusstseinstrübung, Reaktion auf } \\
\text { lautere Kommandos ggf. mit zusätzli- } \\
\text { cher taktiler Stimulation }\end{array}$ & $\begin{array}{l}\text { Bewusstseinstrübung, schwer erweckbar, rea- } \\
\text { giert zielgerichtet auf wiederholte taktile Sti- } \\
\text { mulation und Schmerzreize }\end{array}$ & $\begin{array}{l}\text { Pat. nicht erweck- } \\
\text { bar, selbst nicht auf } \\
\text { Schmerzreize }\end{array}$ \\
\hline Spontanatmung & unbeeinflusst & adäquat & $\begin{array}{l}\text { respiratorische Funktion gering einge- } \\
\text { schränkt }\end{array}$ & $\begin{array}{l}\text { inadäquat, ITN oder } \\
\text { Larynxmaske erfor- } \\
\text { derlich }\end{array}$ \\
& & & &
\end{tabular}




\section{Empfehlung}

Die Art und Intensität der Sedierung sowie die Wahl des Medikaments sollen sich nach der Art des Eingriffs und nach der ASA-Klassifikation sowie dem Risikoprofil des Patienten richten und setzen eine bestimmte räumliche, apparative und personelle Ausstattung voraus.

Liegen die unter „Strukturqualität“ definierten Voraussetzungen nicht vor, soll unter Abwägung von Nutzen, Risiko und Patientenwunsch von einer Sedierung abgesehen werden bzw. bei bestehender Indikation und/oder Patientenwunsch zu einer Sedierung eine Überweisung an eine Einrichtung erfolgen, die diese Voraussetzungen erfüllt.

Empfehlungsgrad: A, Evidenzstärke 5, starker Konsens.

\section{Anmerkungen}

Die American Society of Anesthesiologists und die American Society of Gastroenterologists [19,35] empfehlen, vor Beginn der Untersuchungen eine Risikoabschätzung eventueller kardiovaskulären und respiratorischer Probleme während der Endoskopie vorzunehmen.

Hierzu gehört eine ausführliche Anamnese mit der Frage nach

1. Erkrankungen des kardiovaskulären und respiratorischen Systems, Stridor, Schnarchen, Schlaf-Apnoe-Syndrom,

2. vorausgegangenen Komplikationen bei der Anwendung von Sedativa/Analgetika, regionaler und Allgemein-Anästhesie,

3. Medikamentenallergien, aktueller Medikation und möglichen Medikamenteninteraktionen,

4. Zeitpunkt und Art der letzten Nahrungsaufnahme,

5. Tabak, Alkohol, Drogenkonsum und der körperlichen Untersuchung, welche neben den Vitalparametern die Auskultation von Herz und Lunge beinhaltet.

Die ASA-Klassifikation [36] ( $\bullet$ Tab. 8) sowie die Strukturqualität sind hier Grundlage der bestehenden Leitlinien [9-11, 19, 20, 36-39, 40, 41]. Patienten ab der ASA-Klasse III ( Tab.9) haben ein deutlich erhöhtes Risikopotenzial für eine Sedierung bzw. Intervention.

Das Up-grading des o.a. Statements auf Empfehlungsgrad A, bei Evidenzstärke 5 ergibt sich aus der Evidenzstärke 2b für die aufgeführte ASA-Klassifikation und Ko-Morbidität sowie dem Aspekt der Patientensicherheit.

\subsection{Empfehlung Anästhesie/Intubation}

Empfehlung

Bei Patienten mit höherem Risikoprofil soll die Hinzuziehung eines Anästhesisten erwogen werden, dazu gehören: hohe ASA-Klassifi-

Tab. 8 ASA-Klassifikation.

\begin{tabular}{|ll}
\hline Grad I & kein Risiko \\
\hline Grad II & leichte Erkrankung ohne Leistungseinschränkung \\
\hline Grad III & schwere Erkrankung mit Leistungseinschränkung \\
\hline Grad IV & lebensbedrohliche Erkrankung \\
\hline Grad V & moribund, Tod binnen 1 Tag zu erwarten \\
\hline
\end{tabular}

Tab.9 Charakterisierung der ASA-Klassifikation-III/IV-Stadien durch patientenspezifizierte Risikofaktoren.

\begin{tabular}{|l|}
\hline dekompensierte Herzinsuffizienz \\
\hline koronare Herzerkrankung \\
\hline Herzklappenerkrankung/-ersatz \\
\hline Leber- und Niereninsuffizienz \\
\hline Lungenerkrankungen \\
\hline Gerinnungsstörungen
\end{tabular}

koronare Herzerkrankung

Herzklappenerkrankung/-ersatz

Lungenerkrankungen

Gerinnungsstörungen kation (III-IV) und schwierige endoskopische Eingriffe oder wenn durch pathologisch-anatomische Besonderheiten des Patienten ein erhöhtes Risiko für eine Atemwegsbehinderung während des Eingriffs gegeben ist (z.B. bei kraniofazialen Missbildungen, Tumoren des Zungengrunds, Larynx oder Hypopharynx, massiv eingeschränkter Beweglichkeit der HWS, massiv eingeschränkter Mundöffnung $<3 \mathrm{~cm}$, Mallampatti-Stadien 3 oder 4 bzw. eingeschränkter Kehlkopf-Kinnspitzen Abstand von weniger als $6 \mathrm{~cm}$ ).

Empfehlungsgrad: A, Evidenzstärke 5, Konsens.

\section{Anmerkungen}

Zum Risikoprofil gehören auch pathologisch-anatomische Besonderheiten, die zu respiratorischen Problemen führen können und die eine eventuell notwendige mechanische Atemhilfe bzw. Beatmung erschweren würden. Darüber hinaus geben bestehende Leitlinien weitere Hinweise zur Einschätzung des erhöhten Risikos einer Atemwegsbehinderung [9, 19, 35, 36, 42], bei Patienten mit bereits vorausgehenden Problemen bei einer Anästhesie oder Sedierung.

Dies sind:

1. Patienten mit Stridor, Schnarchen, Schlaf-Apnoe,

2. Patienten mit Fehlbildungen des Gesichtsschädels, wie z.B. Pierre-Robin-Syndrom oder Trisomie-21,

3. Patienten mit Missbildungen des Mundraums, wie kleine Öffnung ( $<3 \mathrm{~cm}$ bei Erwachsenen), Zahnlosigkeit, vorstehende Frontzähne, aus- oder abgebrochene Zähne, hoher gewölbter Gaumen mit Makroglossie, Tonsillenhypertrophie oder nicht sichtbare Uvula,

4. Patienten mit Abnormalitäten des Halses, wie die den Hals und das Gesicht einbeziehende Adipositas, kurzer Hals, eingeschränkte Beweglichkeit des Halses, verminderte HyoidKopf-Distanz ( $<6 \mathrm{~cm}$ bei Erwachsenen), Tumoren des Halses, Erkrankungen oder Traumen der Halswirbelsäule, Trachealveränderungen oder fortgeschrittene rheumatoide Arthritis,

5. Patienten mit Missbildungen des Kiefers, wie Mikrognathie, Retrognathie, Trisomie-Patienten oder ausgeprägte Malokklusion.

6. Auch Menschen mit Alkohol-, Medikamenten- oder sonstigem Drogenabusus sowie höherer ASA-Klassifikation und/ oder mangelnder Kooperationsfähigkeit lassen aufgrund ihres Risikoprofils höhere Anforderungen an die Sedierung erwarten.

\subsection{Empfehlung Schutzintubation \\ Empfehlung}

Eine tiefe Sedierung führt zu Beeinträchtigungen der Schutzreflexe (Schluck-, Hustenreflex). Dies kann Aspirationen Vorschub leisten. Bei besonderen Situationen in der Notfallendoskopie, mit erhöhtem Aspirationsrisiko (z.B. schwere obere gastrointestinale Blutung) unter Sedierung, sollte daher eine tracheale Intubation erwogen werden. Allerdings ist dabei zu berücksichtigen, dass die prophylaktische Intubation mit einem erhöhten Risiko für pneumonische Infiltrate einhergehen kann.

Empfehlungsgrad: B, Evidenzstärke 4, starker Konsens.

\section{Anmerkung}

Grundsätzlich führt eine tiefe Sedierung zur Beeinträchtigung der Schutzreflexe. Bei zusätzlichen Risiken für eine Aspiration, z.B. im Rahmen von Notfallendoskopien bei oberer gastrointestinaler Blutung, erscheint daher eine tracheale Intubation zur Prophylaxe von Aspirationen sinnvoll. Allerdings existieren keine hochwertigen Studien, die ein Überwiegen der Vorteile die- 
ser Maßnahme belegen. Eine grundsätzliche Empfehlung kann daher nicht ausgesprochen werden.

In einer retrospektiv erhobenen Fallkontrollstudie von Koch et al. [43] bei insgesamt 62 Patienten, von denen 42 Patienten im Rahmen einer Varizenblutung vor Beginn der Endoskopie eine prophylaktische endotracheale Intubation erhielten, konnte gezeigt werden, dass nachfolgend bei $17 \%$ der Patienten pneumonische Infiltrate nachweisbar waren, während dies bei den nicht intubierten Patienten nicht der Fall war. Auch lag die Gesamtmortalität bei Patienten mit prophylaktischer Intubation gegenüber nicht intubierten Patienten deutlich höher (21 vs. 5\%). In einer weiteren retrospektiv erhobenen Fallkontrollstudie von Rudoplh et al. [44] an insgesamt 220 Patienten konnte zunächst kein signifikanter Unterschied hinsichtlich des Auftretens von pneumonischen Infiltraten bei prophylaktisch intubierten und nicht intubierten Patienten und der Gesamtmortalität gezeigt werden. Allerdings lag das Auftreten fataler Episoden durch Aspiration mit 2 vs. 0\% höher, wenn keine prophylaktische Intubation erfolgt war.

Aufgrund der unklaren Patientenzuweisung zu beiden Gruppen in solchen retrospektiven Analysen und somit eines möglichen Bias (Intubation bei schwerer kranken Patienten) sind solche Erhebungen jedoch nur von begrenztem Wert.

\subsection{Empfehlung Lagerung \\ Empfehlung}

Analog zum Vorgehen bei der Allgemeinanästhesie bei Operationen soll auch bei sedierten Patienten auf eine korrekte Lagerung zur Vermeidung lagerungsbedingter Schäden geachtet werden. Empfehlungsgrad: A, Evidenzstärke 5, starker Konsens.

\section{Anmerkungen}

Lagerungsschäden spielen bei gastrointestinalen Endoskopien insgesamt nur eine untergeordnete Rolle, sind aber grundsätzlich zu vermeiden.

Vor allem bei flacher Sedierung können sich die Patienten unwillkürlich bewegen und sollten daher angemessen gesichert werden.

Lagerungsschäden treten am ehesten bei Patienten unter der ERCP (Bauch- und Seitenlage) auf bzw. bei Umlagerung vom Untersuchungstisch ins Bett. Direkte Evidenz zur Frage von Lagerungsschäden in der Endoskopie liegt nicht vor, die Empfehlung stützt sich auf die gemeinsame Empfehlung des Berufsverbands Deutscher Anästhesisten und des Berufsverbands der Deutschen Chirurgen [45].

\section{Sedativa|Analgetika/vegetativ wirksame Pharmaka| Kombinationstherapie|Applikationstechniken}

$\boldsymbol{\nabla}$

\subsection{Akzeptanz Patient und Untersucher}

2.1.1. Patientenakzeptanz/Zufriedenheit

Empfehlung

Die Sedierung erhöht den Patientenkomfort und somit die Akzeptanz des endoskopischen Eingriffs. Ideal erscheint hierbei eine Sedierung ohne erinnerliche Missempfindungen und dabei jedoch kurzer Wirkdauer.

Empfehlungsgrad: A, Evidenzstärke 1b, starker Konsens.

\section{Anmerkungen}

Eine Endoskopie unter Sedierung wird in 40-60\% gewünscht [46]. V.a. junge, ängstliche Patientinnen profitieren von einer Sedierung $[27,47]$. Patienten haben in erster Linie den Wunsch nach kompletter Schmerzfreiheit gefolgt von dem Wunsch, möglichst schnell wieder aufzuwachen [48].

In einer Studie von Abraham et al. [49] konnte gezeigt werden, dass Gastroskopien unter Sedierung weniger häufig Wiederholungsuntersuchungen nach sich zogen und mit einer erhöhten Patientenakzeptanz einhergingen. Die Erhöhung der Patientenakzeptanz durch Sedierung bei endoskopischen Eingriffen wurde auch durch andere Studien belegt [50-54].

Allerdings kann es bei der Verwendung von Benzodiazepinen zur Sedierung im Rahmen der Gastrsokopie bei den Patienten zu Beschwerden (insbesondere Brech- und Würgereiz) kommen, die von dem Untersucher u.U. nicht wahrgenommen werden. In einer Studie von Walmsley et al. [55] lag die Rate der vom Untersucher nicht wahrgenommenen o.g. Beschwerden der Patienten bei $12 \%$.

Auch im Rahmen der Koloskopie kann eine „moderate“ Sedierung mit Midazolam in wenigen Fällen zu Schmerzen führen [27], welche vom Untersucher nicht zwangsläufig wahrgenommen werden.

Hingegen zeigt sich bei der Verwendung von Propofol zur Sedierung im Rahmen der ÖGD, Koloskopie und ERCP eine - im Vergleich zu Midazolam - eine gleich gute oder höhere Patientenzufriedenheit ( Tab.10)

\subsubsection{Untersucherzufriedenheit \\ Empfehlung}

Eine Sedierung erhöht die technische Durchführbarkeit und die Vollständigkeit der Untersuchung und steigert somit die Untersucherzufriedenheit (vor allem bei interventionellen Eingriffen). Empfehlungsgrad: A, Evidenzstärke 1b, starker Konsens.

Tab. 10 Patientenzufriedenheit bei Sedierung mit Propofol im Vergleich zum Einsatz von Benzodiazepinen/Opioiden.

\begin{tabular}{|c|c|c|c|c|}
\hline Autor & Untersuchungsart & Sedierungs-Regime & Patientenzahl & Unterschiede \\
\hline $\begin{array}{l}\text { Roseveare } \\
1998[56]\end{array}$ & $\begin{array}{l}\text { patientenkontrollierte Sedierung } \\
\text { mit Propofol bei Koloskopie }\end{array}$ & Diazepam/Pethidin & 66 & Patientenzufriedenheit in beiden Gruppen gleich \\
\hline $\begin{array}{l}\text { Ulmer } \\
2003[57]\end{array}$ & $\begin{array}{l}\text { „Nurse-administered propofol se- } \\
\text { dation“ bei Koloskopie }\end{array}$ & Midazolam/Fentanyl & 100 & $\begin{array}{l}\text { Patientenzufriedenheit in beiden Gruppen ver- } \\
\text { gleichbar ( } 9,3 \text { vs. } 9,4, p>0,5,10 \text {-cm Visuelle-Ana- } \\
\text { log-Skala) }\end{array}$ \\
\hline $\begin{array}{l}\text { Vargo } \\
2002[58]\end{array}$ & $\begin{array}{l}\text { „Nurse-administered propofol se- } \\
\text { dation“ bei ERCP }\end{array}$ & Midazolam/Meperidine & 75 & $\begin{array}{l}\text { Patientenzufriedenheit in beiden Gruppen gleich } \\
\text { gut }\end{array}$ \\
\hline $\begin{array}{l}\text { Sipe } \\
2002[59]\end{array}$ & $\begin{array}{l}\text { „Nurse-administered propofol se- } \\
\text { dation“ bei Koloskopie }\end{array}$ & Midazolam & 80 & $\begin{array}{l}\text { Patientenzufriedenheit unter Propofol signifikant } \\
\text { höher ( } 9,3 \text { vs. } 8,6 ; p<0,05,10 \text {-Punkte visuelle } \\
\text { Analogskala) }\end{array}$ \\
\hline $\begin{array}{l}\text { Weston } \\
2003[60]\end{array}$ & $\begin{array}{l}\text { „Nurse-administered propofol se- } \\
\text { dation“ bei ÖGD bei Pat. mit Le- } \\
\text { berzirrhose }\end{array}$ & Midazolam & 20 & $\begin{array}{l}\text { Patientenzufriedenheit unter Propofol signifikant } \\
\text { höher }(p<0,05)\end{array}$ \\
\hline
\end{tabular}


Tab.11 Vergleich der Vitalparameter bei Sedierung mittels Propofol versus Midazolam/Dolantin (bzw. Meperidin) im Rahmen der ERCP (ns = nicht signifikant).

\begin{tabular}{|c|c|c|c|c|}
\hline Autor & Vitalparameter & Propofol & Midazolam/Pethidin (Meperidin) & Unterschiede \\
\hline \multirow[t]{3}{*}{ Vargo J] [58] } & $\mathrm{SpO}_{2}<90 \%$ & $21 / 37(57 \%)$ & $14 / 38(37 \%)$ & ns \\
\hline & RR $<75 \%$ vom Ausgangswert & $7 / 37(18,9 \%)$ & $6 / 38(15,8 \%)$ & ns \\
\hline & $\mathrm{HF}<75 \%$ vom Ausgangswert & $3 / 37(8,1 \%)$ & $0 / 38(0,0 \%)$ & ns \\
\hline \multirow[t]{4}{*}{ Riphaus A [121] } & $\mathrm{SpO}_{2}<90 \%$ & $7 / 75(9,0 \%)$ & $8 / 75(11 \%)$ & ns \\
\hline & mittlerer Abfall der $\mathrm{SpO}_{2}$ & $3 \%(2 \%)$ & $6 \%(3 \%)$ & $<0,01^{1}$ \\
\hline & $\mathrm{RR}<90 \mathrm{mmHg}$ & $4 / 75(5,3 \%)$ & $6 / 75(8 \%)$ & ns \\
\hline & $\mathrm{HF}<50 /$ Min. & $4 / 75(5,3 \%)$ & $3 / 75(4 \%)$ & ns \\
\hline \multirow[t]{4}{*}{ Wehrmann T[115] } & $\mathrm{SpO} 2<90 \%$ & $8 / 98(8,2 \%)$ & $11 / 99(11 \%)$ & ns \\
\hline & mittlerer Abfall der $\mathrm{SpO}_{2}$ & $3 \%(2 \%)$ & $5 \%(3 \%)$ & $<0,01^{1}$ \\
\hline & $\mathrm{RR}<90 \mathrm{mmHg}$ & $2 / 98(2,0 \%)$ & $7 / 99(7,1 \%)$ & ns \\
\hline & $\mathrm{HF}<50 /$ Min. & $2 / 98(2,0 \%)$ & $5 / 99(5,1 \%)$ & ns \\
\hline \multirow[t]{3}{*}{ Krugliak P [120] } & $\mathrm{n}$ & 14 & 15 & \\
\hline & RR $<20 \%$ vom Ausgangswert & $37,0 \pm 30,1$ & $25,2 \pm 18,6$ & ns \\
\hline & $\mathrm{HF}<20 \%$ vom Ausgangswert & $48,2 \pm 38,0$ & $14,6 \pm 25,0$ & $<0,01^{1}$ \\
\hline \multirow[t]{4}{*}{ Jung M [66] } & $\mathrm{n}$ & 40 & 40 & \\
\hline & Abfall Sp02 (\%) & -2 & -4 & ns \\
\hline & mittlerer RR Abfall (\%) & 14 & 17 & ns \\
\hline & Anstieg der HF (\%) & $+3,5$ & +2 & ns \\
\hline
\end{tabular}

1 Signifikant $(p<0,01)$.

\section{Empfehlung}

Bei interventionellen Endoskopien ist Propofol hinsichtlich der Untersucherzufriedenheit dem Midazolam überlegen.

Empfehlungsgrad: B, Evidenzstärke 2b, starker Konsens.

\section{Anmerkungen}

Die Sedierung mit Midazolam im Rahmen der ÖGD kann zwar zu einer hohen Patientenakzeptanz [61], bei jedoch unzufriedenem Untersucher führen [62]. Durch Kombination mit einem Opiat kann die Untersucherzufriedenheit verbessert werden.

So zeigten die Ergebnisse der randomisierten Studie von Laluna et al. [63] an 107 Patienten, die zur ÖGD entweder mittels Midazolam/Placebo oder Midazolam/Meperidin sediert wurden, dass die Untersucherzufriedenheit bei Verwendung des Kombinationsregimes signifikant besser $\operatorname{war}(\mathrm{p}<0,001)$. Für die Patientenakzeptanz konnte hingegen kein wesentlicher Unterschied gezeigt werden.

Bei Verwendung von Propofol konnte hinsichtlich der Patiententoleranz bzw. Untersucherzufriedenheit in einer Studie von $\mathrm{Ng}$ [64], in der 80 Patienten randomisiert eine PCS mit Propofol oder Midazolm zur Koloskopie erhielten, eine signifikant höhere Untersucherzufriedenheit bei der Verwendung von Propofol $(p=0,01)$ gezeigt werden.

Insbesondere bei länger dauernden und komplexeren Eingriffen ist eine tiefe Sedierung in höherem Maße erforderlich [65], um die Untersuchung ohne ungewünschte und unkontrollierbare Spontanbewegungen des Patienten sicher durchführen zu können.

In zwei Studien von Jung et al. [66] und Wehrmann et al. [67], in der die Patienten zur ERCP randomisiert eine Sedierung mittels Propofol oder Midazolam erhielten, zeigte sich eine signifikant höhere Untersucherzufriedenheit bei der Verwendung von Propofol.

Mit zunehmender Tiefe der Sedierung, durch höhere Dosen der verschiedenen Substanzen, steigt aber auch die Häufigkeit von unerwarteten/unerwünschten Nebenwirkungen (siehe auch Dosierungsempfehlungen der Fachinformationen der verschiedenen Hersteller und $\odot$ Tab. 11, Abschnitt 2.2.3.1.).

\subsection{Monotherapien}

\subsubsection{Propofol}

\subsubsection{Allgemeines}

Propofol ist ein Sedativum mit minimalen analgetischen Effekt. Der sedierende Effekt von Propofol beruht auf einer Akkumulation von Gamma-Amino-Buttersäure (GABA) durch eine verminderte Dissoziation des GABA-Rezeptors. Die genauen Wirkmechansimen von Propofol sind im Detail bis heute jedoch nicht vollständig geklärt. Propofol ist hochgradig lipophil und entfaltet seine Wirkung innerhalb von $30-45 \mathrm{~s}$. Aufgrund der raschen hepatischen Metabolisierung dauert der sedierende Effekt nur 4- 8 min nach Applikation. Wegen dieser Eigenschaften (rascher Wirkungseintritt bei kurzer Wirkdauer) ist es für die gastrointestinale Endoskopie ein geeignetes Sedativum [6-8, 59, 60, 68 - 70]. Die Wirkung von Propofol ist individuell in Abhängigkeit vom Alter [71, 72], Körpergewicht, Begleiterkrankungen oder Begleitmedikation unterschiedlich. Mitunter können mit einer einzigen Dosis verschiedene Sedierungstiefen ( $\bullet$ Tab.6, 7) durchschritten und eine kurzfristige Apnoe ausgelöst werden [73]. Im Gegensatz zu Midazolam existiert für Propofol allerdings kein Antagonist. Alle Endoskopie-Teams, die mit Propofol sedieren, müssen deshalb in der Lage sein, einen Atemstillstand kurzfristig zu beherrschen (s. Kap. 4. Prozessqualität).

Neben Hypoxämien können bei der Anwendung von Propofol typischerweise Hypotonien auftreten [12] ( $\bullet$ Tab. 11, Abschnitt 2.2.3.1.)

Raritäten sind Bakteriämien bis zur Sepsis bei unsachgemäßer Lagerung sowie in Einzelfällen Pankreatitiden [74, 75]. Ferner ist ein „Propofol-Infusions-Syndrom“ (mit Rhabdomyolyse) beschrieben, welches bisher jedoch nur bei langdauernder Applikation $(>48 \mathrm{~h})$ in hoher Dosierung $(>4 \mathrm{mg} / \mathrm{kg} / \mathrm{h})$ aufgetreten ist [76]. Propofol ist bei bekannter Allergie gegen Hühnereiweiß, Sojaeiweiß oder Sulfit kontraindiziert.

Die Applikation von Propofol erfolgt als initiale Bolusgabe zur Einleitung der Sedierung mit anschließender Aufrechterhaltung der Sedierung durch repetitive Bolusgabe oder mittels kontinuierlicher Applikation via Perfusor. 


\subsubsection{Techniken der Propofol-Applikation}

Alternative Applikationsformen zu der, derzeit in der deutschen Endoskopie gebräuchlichsten, intermittierenden Bolusgabe stellen die Perfusor-Applikation (mit initialem Einmalbolus zur Einleitung), die sog. „Target-controlled-Infusion“ (TCI), die patientenkontrollierte Sedierung (PCS) und die sog. „Computer-Assisted-Personalizied Sedation“ (CAPS) dar. Hierbei sind die intermittierende Bolusapplikation und die Perfusor-Applikation als Standardverfahren anzusehen, während die übrigen Verfahren zumindest hinsichtlich ihres Einsatzes in der Endoskopie noch experimentellen Charakter haben.

\subsection{Intermittierende Propofol-Bolusapplikation}

Hierbei wird zur Einleitung ein Gewichts- und ggf. auch Altersoder Komorbiditäts-adaptierter Bolus (z.B. $40 \mathrm{mg}$ bei $<70 \mathrm{~kg}$ KG bzw. $60 \mathrm{mg}$ bei $\geq 70 \mathrm{~kg} \mathrm{KG}$ ) intravenös appliziert und nachfolgend bedarfsadaptiert repetitive Boli von z.B. 10-20 mg zur Aufrechterhaltung der gewünschten Sedierungstiefe verabreicht. Gegebenfalls kann zur Einleitung zusätzlich ein Benzodiazepin und/oder Opiat hinzugefügt werden (s. Kapitel 2.3. Kombinationstherapie). Die intermittierende Bolusapplikation wurde nahezu in allen publizierten Studien zur Frage der Sedierungseffizienz von Propofol bei endoskopischen Untersuchungen/Behandlungen im Vergleich mit anderen Pharmaka (z.B. Midazolam) eingesetzt und ist daher zurzeit in der Endoskopie die bestdokumentierte und derzeit auch gebräuchlichste Applikationsweise.

\subsection{Kontinuierliche Propofol-Applikation mittels Perfusor- systemen}

Hierbei wird gleichfalls ein Gewichts- und ggf. auch Altersoder Komorbiditäts-adaptierter Bolus von Propofol (ggf. auch kombiniert mit anderen Pharmaka) zur Einleitung der Sedierung gegeben. Danach wird die Sedierung durch eine (in der Regel) gewichtsadaptierte Propofol-Dauerinfusion aufrechterhalten. Die Dosierung richtet sich auch nach der gewünschten Sedierungstiefe und dem individuellen Risikoprofil des Patienten. Die meisten Systeme erlauben die zusätzliche bedarfsadaptierte Gabe von Propofol-Boli. Spezielle Perfusorsysteme für die Anästhesie errechnen nach Eingabe verschiedener Patientenparameter (z.B. Gewicht, Größe, Alter) die Erhaltungsdosis von Propofol 1\%- oder 2\%-Lösung (nach Vorgabe der gewünschten Sedierungstiefe) selbstständig.

Die Perfusor-Applikation von Propofol ist derzeit die gebräuchlichste Methode zur Aufrechterhaltung der total-intravenösen Anästhesie (TIVA) in Deutschland. Für den Einsatz in der Endoskopie liegen dagegen bisher nur wenige Studien vor [77], ein randomisierter Vergleich zwischen Perfusor-Applikation und intermittierender Bolusgabe im Bereich der Endoskopie existiert unseres Wissens nicht.

\section{Empfehlung}

Die intermittierende Bolusapplikation von Propofol kann derzeit als Standardverfahren zur Anwendung von Propofol in der Endoskopie angesehen werden. Die Propofol-Gabe mittels Perfusor ist in der Anästhesie sehr umfassend dokumentiert und gilt als Standard für die total-intravenöse Anästhesie, hinsichtlich des Einsatzes in der Endoskopie liegen bisher jedoch nur wenige publizierte Daten vor.

Empfehlungsgrad A, Evidenzstärke 2b, starker Konsens.

\subsection{Patientenkontrollierte (Analgo-)Sedierung (PCS)}

Die patientenkontrollierte Abgabe von Medikamenten stammt ursprünglich aus der Schmerztherapie und gehört heute in der postoperativen Analgesie zum Standard (z.B. im Konzept der sog. „Fast-track surgery“). Der Patient kann sich selbst durch Druck auf eine Taste eine bestimmte Dosis eines Medikaments mithilfe einer programmierbaren Infusionspumpe intravenös verabreichen. Die wiederholte Gabe kann optional nur zeitlich verzögert gestattet werden (sog. „Lockout“-Mechanismus), um eine Überdosierung zu vermeiden.

Die PCS mag insbesondere bei solchen endoskopischen Untersuchungen sinnvoll sein, wo relativ kurzdauernde Schmerzepisoden vom Patienten zu tolerieren sind, wie es z.B. bei der Koloskopie öfters der Fall ist (z. B. Sigmapassage, Flexurenpassage). Hierfür ist die Gabe besonders kurz wirksamer Pharmaka über diese Systeme (z.B. Graseby-3000-Pumpe) ideal, häufig wird hier eine Kombination von Propofol mit kurz wirksamen Opiaten (z. B. Alfentanyl) verwendet.

So führte der Einsatz eines PCS-Systems (Propofol plus Alfentanyl) in einer randomisierten Studie gegenüber Midazolam und Meperidin [78] und zwei randomisierten Vergleichsstudien gegenüber Diazepam und Meperidin (in Deutschland als Pethidin bezeichnet) zu einer vergleichbaren Patientenzufriedenheit [56, 79], in zwei weiteren Studien gegenüber Midazolam gar zu einer höheren Patientenzufriedenheit $[64,77]$. In einer anderen randomisierten Studie wurde jedoch unter Verwendung von PCS ein erhöhter Schmerz-Score als unter Midazolam dokumentiert [78]. Unter PCS mit Propofol traten jedoch weniger unerwünschte Ereignisse (Sauerstoffuntersättigung oder Blutdruckabfall) als unter Diazepam auf $[56,79]$. In 2 dieser Studien waren 97 bzw. $78 \%$ der mittels PCS-Technik bei der Koloskopie sedierten Patienten bereit, sich, falls nötig, mit der gleichen Methode wieder sedieren zu lassen [80, 81]. Jüngeres Alter, weibliches Geschlecht und niedrigere Patientenzufriedenheit waren unabhängige Faktoren für die Ablehnung des PCS-Verfahrens.

In einer Schweizer Studie zum Thema PCS verweigerten 35\% aller angesprochenen Probanden die Studienteilnahme, da sie entweder einen vollständigen Bewusstseinsverlust wünschten oder selbst nicht die Verantwortung für die Medikamentengabe übernehmen wollten [13]. In dieser Studie konnte aber bei den teilnehmenden Probanden eine signifikant niedrigere Propofol-Dosis unter Einsatz der PCS, wie unter einer intermittierenden Bolusgabe (durch eine Schwester, s. NAPS), bei der Koloskopie beobachtet werden [13].

\section{Empfehlung}

Bei Verwendung von Propofol mit oder ohne Opiat zur Koloskopie kann eine patientenkontrollierte Sedierung als Alternative zur Bolusgabe durchgeführt werden.

Empfehlungsgrad: 0, Evidenzstärke 2b, starker Konsens.

\subsection{Target-Controlled Infusion (TCI)}

Das Prinzip ermöglicht die intravenöse Gabe von Propofol (oder auch anderen Pharmaka) mittels Infusionspumpe, wobei Dosierung und Infusionsgeschwindigkeit computergestützt gesteuert wird [82]. Das Computersystem kalkuliert hierbei die individuelle, von der Infusionspumpe abzugebende Infusionsrate, die nötig ist, um eine voreingestellte, gewünschte Medikamentenkonzentration im Blut aufrechtzuerhalten, anhand vorgegebener Algorithmen, die verschiedene patientenseitige Parameter berücksichtigen (z.B. Alter, Geschlecht, Größe, Gewicht, Sedierungstiefe etc.). Nachdem die initiale Dosierung zum Erreichen 
der gewünschten Blutkonzentration errechnet wurde, erfolgt dann im Verlauf eine entsprechende Anpassung der Infusionsrate (hierbei wird die gewünschte Blutkonzentration vom Arzt vorgegeben, sog. „offenes System“).

Zusätzlich kann bei speziellen Systemen die Infusionsgeschwindigkeit darüber hinaus auch anhand von Muskeltonus, auditorisch evozierten Potenzialen und/oder anderen physiologischen Parametern, welche die Sedierungstiefe reflektieren, variiert werden, um die Sedierung zu steuern (sog. „geschlossene Systeme“).

Der potenzielle Vorteil der TCI-Methode gegenüber einer reinen Dauerinfusion (mit fixierter Dosis und Infusionsgeschwindigkeit) besteht in der Möglichkeit, eine Akkumulation des applizierten Pharmakons zu vermeiden (da die Infusionsgeschwindigkeit fortlaufend variiert wird).

In einer Evaluation von Fanti et al. [83] an 205 Patienten, welche in tiefer Sedierung (ohne mechanische Ventilation) einer ERCP unterzogen wurden, erfolgte unter Verwendung eines offenen TCI-Systems die Propofol-Applikation initial mit einer Zielkonzentration von $4 \mathrm{ug} / \mathrm{ml}$, welche dann im Verlauf zwischen 2 - 5 ug $/ \mathrm{ml}$ durch den Anästhesisten gehalten wurde. Zusätzlich war die Bolusapplikation von Fentanyl (50 - 100 ug i.v.) möglich. Seitens des Endoskopikers wurde die Sedierung in 201/205 Fällen als exzellent eingestuft, es wurden nur 4 Fälle mit Hypoxämie $\left(\mathrm{pO}_{2}<85 \%\right)$ und in einem Fall die Notwendigkeit einer Maskenbeatmung gesehen.

Bei Verwendung eines geschlossenen TCI-Systems, wobei die Infusionsrate EEG-gestützt (mittels Bestimmung des bispektralen Index, BIS) kontrolliert wurde, wurde bei 16 Patienten eine Koloskopie durchgeführt [84]. Im Median wurde in dieser Studie von Leslie et al. [84] eine Propofol-Konzentration von $2,3 \mathrm{ug} / \mathrm{ml}$ angesteuert, wobei überwiegend ein BIS-Level von 80 beobachtet wurde (entspricht einer leichten bis moderaten Sedierungstiefe).

Weitere Studien eruierten den kombinierten Einsatz von TCI und PCS, wobei die Applikationsrate der TCI-Pumpe durch die Betätigung einer Handtaste durch den Patienten modifiziert werden konnte. Hierbei wurden, bei allerdings kleinen Fallzahlen $(n=20-40)$, günstige Sedierungseffekte während Koloskopie oder ERCP dokumentiert [84-87]. In der Studie von Stonell et al. [87] erfolgte ein randomisierter Vergleich des TCI/PACSSystems mit der repetitiven Bolusgabe von Propofol bei der Koloskopie $(n=40)$ durch einen Anästhesisten, wobei sich keine signifikanten Unterschiede hinsichtlich Sedierungseffizienz und Komplikationsrate fanden, auch wenn die Gesamt-Propofol-Dosis in der TCI-Gruppe tendenziell niedriger war wie in der Bolusgruppe (233 vs. $288 \mathrm{mg}, \mathrm{p}=0,05$ ).

\subsection{Computer-Assisted Personalized Sedation (CAPS)}

Dieses Verfahren erweitert die TCI-Gabe von Propofol um eine zusätzliche Implementierung sowohl von physiologischen Monitoring-Parametern (Pulsfrequenz, Blutdruck, $\mathrm{O}_{2}$-Sättigung und kapnografische Bestimmung der $\mathrm{CO}_{2}$-Exhalation) wie von Patienten-Reaktionen auf gezielte verbale (über Kopfhörer) und taktile (via Vibrationsmaus) Stimuli. Somit wird die Sedierung vollständig computergestützt gesteuert durchgeführt und überwacht. Das System ermöglicht derzeit nur die Steuerung einer moderaten Sedierungstiefe, tiefe Sedierungen oder Narkosen sind derzeit nicht vorgesehen. Eine Zulassung für dieses System zum klinischen Einsatz liegt derzeit in Europa nicht vor.
In einer ersten bi-zentrischen Evaluation in USA und Belgien konnte bei insgesamt 96 Patienten, bei denen eine Gastroskopie oder Koloskopie durchgeführt wurde, ein ausreichender Sedierungseffekt ohne Komplikationen beobachtet werden. Nach initialer Bolusgabe von Fentanyl (25-100 ug) wurden zwischen 20 - $350 \mathrm{mg}$ (Median $70 \mathrm{mg}$ ) Propofol über das System verabreicht [88].

\section{Empfehlung}

TCI und CAPS wurden in einigen Studien geprüft; aufgrund der existierenden Datenlage kann zum Stellenwert dieser Verfahren zurzeit keine Aussage gemacht werden.

Empfehlungsgrad 0, Evidenzstärke 1b, starker Konsens.

\subsubsection{Benzodiazepine}

Benzodiazepine induzieren eine Anxiolyse, Amnesie und Sedierung und wirken parallel antikonvulsiv und muskelrelaxierend. Die Wirkung erklärt sich über eine Bindung dieser Substanzen an den $\mathrm{GABA}_{\mathrm{A}}$-Rezeptor. Unterschiedliche Benzodiazepine können jedoch unterschiedliche pharmakologische Eigenschaften aufweisen (z. B. stärker sedierender Effekt oder stärker anxiolytischer Effekt) [89].

\subsubsection{Diazepam}

Diazepam war zu Beginn der Endoskopie das einzig verfügbare Sedativum, ist aber inzwischen bei endoskopischen Untersuchungen in westlichen Ländern nur noch selten in Gebrauch. Dies beruht auf der vergleichsweise langen Halbwertszeit im Gegensatz zu nachfolgenden kurz wirksamen Benzodiazepinen wie Midazolam [90-92]. An Nebenwirkungen kann es vorwiegend bei Diazepam zu Atemdepression [93], Husten und Dyspnoe kommen. Weiterhin kann eine Phlebitis an der Injektionsstelle auftreten, insbesondere bei Verwendung wasserlöslicher Darreichungsformen [94]. Die übliche Dosis ist die Einmalgabe von 5-10 mg (siehe auch Dosierungsempfehlungen der Fachinformationen der verschiedenen Hersteller).

\subsubsection{Midazolam}

Midazolam ist als kurz wirkendes Benzodiazepin, das bis heute wohl am häufigsten verwandte Sedativum in der Endoskopie [95]. Die sedierende Potenz ist um den Faktor 1,5- bis 3,5-mal größer als bei Diazepam [96]. Die Substanz wirkt nach $1-3 \mathrm{~min}$, das Wirkmaximum ist nach 3-4 min erreicht, obgleich die Wirkungsdauer 15-80 min anhält [97] und von Kofaktoren wie Übergewicht, fortgeschrittenes Alter und Erkrankungen der Leber und Niere abhängig ist. Das Nebenwirkungsprofil entspricht dem des Diazepams, jedoch treten Phlebitiden seltener auf [98]. Wie gelegentlich auch bei anderen Benzodiazepinen kann nach Midazolam-Gabe selten eine paradoxe Reaktion, gekennzeichnet durch Aggressivität, Feindlichkeit und Unruhe, auftreten. Diese wird bei kurz dauernder oraler Anwendung mit ca. 5\% beschrieben [99]. In einer Studie von Christe et al. [100] zur Sedierung mit Midazolam bei älteren Patienten (mittleres Alter $84 \pm 7 \mathrm{~J}$ ) während der ÖGD zeigten sich bei $14 \%$ der Patienten noch am Folgetag Verwirrtheitszustände.

Bei der Gastroskopie wird Midazolam gewöhnlich als Bolus von $30-80 \mu g / k g$ KG verabreicht [6, 7, 100, 101]. Bei der Koloskopie wird meist ein Anfangsbolus zwischen 30 und $50 \mu \mathrm{g} / \mathrm{kg}$ mit nachfolgenden Boli geringerer Dosis bis zur nötigen Sedationstiefe $[64,102,103]$ gegeben, wobei ab einem Alter von über 60 Jahren geringere Dosierungen empfohlen werden [79, 100, 103-105]. 
Prinzipiell ist bei Sedierungswunsch die Verabreichung von Midazolam vor Untersuchungsbeginn günstiger als die Gabe nach Bedarf während der Untersuchung $[106,107]$.

\subsubsection{Antagonisierung der Wirkung von Midazolam durch} Flumazenil

Die Wirkung von Midazolam kann durch den Benzodiazepinspezifischen Antagonisten Flumazenil aufgehoben werden $[108,109]$. In einer Studie von Mora et al. [110] konnte gezeigt werden, dass Flumazenil stärker in der Antagonisierung von Benzodiazepin induzierter Sedierung und Amnesie als in der respiratorischen Hypoventilation ist. Die Aufhebung der Midazolam-induzierten Atemdepression erfolgt 120 s nach intravenöser Flumazenilgabe [111].

Die Halbwertszeit von Flumazenil beträgt $0,7-1,3 \mathrm{~h}$, mit einer durchschnittlichen Dauer des antogonisiernenden Effekts von $1 \mathrm{~h}$. Da der Effekt von Midazolam 80 min oder länger andauern kann, besteht die Gefahr der Re-Sedierung, sodass erneute Flumazenil-Gaben notwendig werden können.

In einer Studie von Andrews et al. [112], erhielten 50 Patienten nach Gastroskopie mittels Midazolam-Sedierung, unmittelbar nach der Untersuchung und 30 min später, entweder Flumazenil oder Placebo. Patienten, die Flumazenil erhielten, zeigten bereits 5 min später eine deutliche Verbesserung des Erinnerungsvermögens, der Psychomotorik und der Koordination $(\mathrm{p}<0,001)$. Eine erneute Re-Evaluation der gleichen Parameter nach 3,5 h konnte jedoch keinen Unterschied in den beiden Gruppen zeigen. Hingegen zeigten die Ergebnisse der Studie von Bartelsman et al. [113] an 69 Patienten, die nach Midazolamgabe zur ÖGD Flumazenil oder Placebo erhielten, keinen Hinweis für eine ReSedierung innerhalb von $6 \mathrm{~h}$.

Die routinemäßige Gabe von Flumazenil nach Beendigung einer Untersuchung reduziert zwar die Aufwachzeit [114], eindeutige Vorteile für den Patient und den Untersucher konnten bisher jedoch nicht dokumentiert werden.

Auch ist Vorsicht bei Patienten mit bestehender Carbamazepin-, hoch dosierter Medikation mit trizyklischen Antidepressiva oder chronischem Benzodiazepinabusus geboten, da es hier zu Krampfanfällen oder Entzugserscheinungen kommen kann. Die routinemäßige Verwendung von Flumazenil kann daher nicht empfohlen werden.

Bei Patienten, bei denen die Anwendung von Flumazenil dennoch nötig werden sollte, ist hier auf einen entsprechend längeren Überwachungszeitraum zu achten.

\subsubsection{Amnesie - Diazepam versus Midazolam Empfehlung}

Mit Ausnahme von gelegentlichen, venösen Komplikationen weist Diazepam keine Nachteile gegenüber Midazolam auf. Wenn wegen der gewünschten stärkeren Amnesie mit Benzodiazepinen sediert werden soll, ist Midazolam dem Diazepam wegen der kürzeren Halbwertszeit vorzuziehen.

Empfehlungsgrad B: Evidenzstärke IIa, Konsens.

\section{Anmerkungen}

Die Amnesie nach Midazolam ist eindeutig untersucht [123] und in allen Studien signifikant nachgewiesen. Wird eine Amnesie nicht gewünscht, muss auf Midazolam verzichtet werden. Eine Alternative als Benzodiazepin ist das Diazepam [91, 92]. Die längeren Halbwertszeiten von Diazepam haben sich in Studien nicht als nachteilig gegenüber Midazolam dargestellt [92, 96, 124]. Allerdings war der Patientenkomfort in einigen Studien nach Diazepam geringer als nach Midazolam. Unter dem Gesichtspunkt Amnesie als Nebenwirkung weist Midazolam die höchste Potenz dieser NW aller untersuchten Sedierungskonzepte auf.

\subsubsection{Propofol versus Midazolam}

Empfehlungen

Die Daten zu Wirkungsprofil und Komplikationen legen den Schluss nahe, Propofol bevorzugt vor Midazolam bei Sedierungsbedarf in der gastrointestinalen Endoskopie zu verwenden. Individuelle Abstimmungen auf die Patientensituation und den Untersuchungstyp sowie die Erfüllung der in dieser Leitlinie genannten persönlichen, personellen, apparativen und strukturellen Voraussetzungen sind jedoch erforderlich.

Empfehlungsgrad: A, Evidenzstärke 2b, starker Konsens.

\section{Anmerkungen}

Bezüglich der Patientenakzeptanz bzw. -zufriedenheit ergibt sich aus der Zusammenfassung der vorliegenden randomisierten Studien ( Tab. 10), dass diese bei der Sedierung mit Propofol im Vergleich zu Benzodiazepin von den Untersuchern als besser $[59,60]$ oder gleich gut [56-58] beurteilt wird.

Randomisierte Studien legen den bevorzugten Gebrauch von Propofol für ÖGD, Koloskopie und ERCP nahe [6-8, 58-60, $66-70,115]$. Vorteile bei der Sedierung mittels Propofol im Vergleich zu Benzodiazepinen, sowohl für den Patienten als auch den Untersucher liegen in der kürzeren Zeit bis zum Wirkungseintritt [59], der - insbesondere bei interventionellen Endoskopien (wie der ERCP) - signifikant besseren Patientenkooperation [66, 67, 115] sowie der schnelleren Erholungszeit für die Patienten $[6-8,58-60,66-70]$, auch hinsichtlich der psychomotorischen Funktionen [8].

Die Überlegenheit von Propofol hinsichtlich der Erholung der neuropsychologischen/kognitiven Funktionen im Vergleich zur Gabe von Midazolam/Fentanyl konnte zunächst in zwei Studien im Rahmen der ambulanten Koloskopie gezeigt werden [57, 58]. Auch in einer randomisierten Studie von Riphaus et al. [8] zur Erholung der psychomotorischen Funktionen (ermittelt anhand eines Fahrsimulators) nach Sedierung mittels Propofol im Vergleich zu Midazolam/Dolantin zur ÖGD/Koloskopie, zeigte sich bei der Verwendung von Propofol neben einer signifikant schneller Aufwachzeit auch eine deutliche raschere Erholung der psychomotorischen Funktionen.

Ein weiterer Vorteil bei der Verwendung von Propofol im Vergleich zu Midazolam liegt in der möglicherweise besseren Qualität der Untersuchungen.

Hierzu konnte in einer Studie von Meining et al. [116], in der die Untersuchungsqualität bei der ÖGD unter Sedierung mittels Propofol vs. Midazolam verglichen wurde, gezeigt werden, dass bei Verwendung von Propofol die Darstellungsqualität des oberen Gastrointestinaltrakts (auf Videoaufnahmen) verbessert wurde. Allerdings war die endoskopische Untersuchungszeit in der Propofol-Gruppe länger wie in der Midazolam-Gruppe [116]. Bei der Koloskopie erleichtert die Sedierung mit Propofol die Untersuchung [117], wobei eine moderate Sedierung („conscious sedation“) bei Koloskopien in der Regel ausreicht [118].

\subsubsection{Kardiorespiratorische Komplikationen}

Die vergleichenden Daten zu den Komplikationen wurden in einer Metaanalyse von Quadeer et al. [119] aus 12 randomisierten Studien zusammengestellt: Hier wurde das relative Risiko einer Sedierung mit Propofol gegenüber Benzodiazepinen 
beschrieben. Hierbei war der Einsatz von Propofol bei der Koloskopie mit signifikant weniger Nebenwirkungen assoziiert. Bei anderen endoskopischen Eingriffen (ÖGD, ERCP) war kein signifikanter Unterschied feststellbar.

Bei Verwendung von Propofol im Rahmen der ERCP zeigt sich jedoch ein im Vergleich zur Sedierung mittels Midazolam/Dolantin bzw. Meperidin teils signifikant erhöhtes Risiko für das Auftreten einer arteriellen Hypotonie [58, 66, 115, 120, 121] und auch ein Abfall der Sauerstoffsättigung unter 90\% wird bei Sedierung mittels Propofol tendenziell, wenn auch nicht signifikant, häufiger beobachtet ( $\bullet$ Tab.11).

In einer kürzlich veröffentlichten Risikofaktoren-Analyse von Wehrmann und Riphaus [122] bei 9547 Patienten, die über einen 6-Jahres-Zeitraum eine Propofolsedierung im Rahmen der interventionellen oberen Endoskopie (ÖGD, $n=5374$, ERCP, $n=3937$, EUS, $n=236$ ) erhielten, hatten 3151 Patienten eine Propofolmonosedierung und 6396 Patienten eine Kombinationstherapie mit Propofol/Midazolam. Insgesamt wurden 135 schwerwiegende Komplikationen, die $\mathrm{zu}$ einem vorzeitigen Abbruch der Untersuchung führten, dokumentiert (1,4\%). Eine kurzfristige Maskenbeatmung war bei 40 Patienten $(0,4 \%)$ und eine endotracheale Intubation bei 9 Patienten $(0,09 \%)$ notwendig. Acht Patienten bedurften einer weiteren Überwachung auf Intensivstation (0,3\%), 4 Patienten verstarben, in 3 Fällen im Rahmen potenziell auch sedierungsassoziierter Nebenwirkungen (Mortalitätsrate $0,03 \%$ ). Nach multivarianter Analyse der Daten waren Notfalluntersuchungen und eine höhere PropofolDosis als unabhängige Risikofaktoren für das Auftreten von kardiorespiratorischen Komplikationen zu werten [122].

\subsubsection{Sonstige Pharmaka als Monotherapeutika}

\subsubsection{Einleitung}

Unter sonstige Pharmaka sind weitere - nicht den gängigen Substanzklassen zuzuordnende -, entweder sedierend/hypnotisch oder analgetisch wirksame Substanzen zu nennen. Hierunter fallen Opiate und Ketamin (als Monotherapeutika), Inhalationsanästhetika, nicht steroidale Antiphlogistika sowie die Neuroleptanalgesie. Aus der Vergangenheit gibt es zu diesen Substanzen nur wenige Studien, die mit mäßigem Evidenzgrad die prinzipielle Eignung dieser Pharmaka zur Sedierung aufgezeigt haben. Unter Bewertung der vorhandenen u.a. Literatur fällt auf, dass

1. die Häufigkeit unerwünschter Nebenwirkungen erheblich höher ist als bei den gebräuchlichen Konzepten.

2. einige dieser Konzepte (wie z.B. Neurolept-Analgesie) auch in der modernen Anästhesie keinen Stellenwert mehr haben.

3. zu Ketamin die wissenschaftliche Evidenz fehlt; spezifische Nebenwirkungen lassen die Anwendung von Ketamin als Monosubstanz als nicht sinnvoll erscheinen. Bezüglich der Anwendung von Ketamin im Rahmen von Kombinationstherapien (z.B. in Kombination mit Midazolam oder Propofol) liegen nur wenige Studien mit geringer Fallzahl vor, die einen positiven Effekt vermuten lassen. Hier bedarf es jedoch der weiteren Evaluation in randomisierten Studien mit entsprechend hoher Fallzahl.

4. bezüglich Inhalationsanästhetika zu beachten ist, dass diese besondere Geräte, besondere Überwachungsverfahren und Anforderungen an die Sicherheit der Arbeitsplätze erfordern. Da bei der Endoskopie stets von offenen Inhalationssystemen ausgegangen werden kann, ist die Einhaltung von MAC-Werten der verwendeten Substanzen nicht möglich, insbesondere da eine routinemäßige Sicherung des Atemwegs (Intubation, dichte Larynxmaske) bei der Endoskopie ungebräuchlich ist.

5. für einen routinemäßigen Einsatz von NSAR im Rahmen endoskopischer Untersuchungen aufgrund der aktuellen Datenlage keine hinreichende Evidenz gegeben ist.

\section{Empfehlung}

Opiate, Ketamin, Inhalationsanästhetika oder die Neuroleptanalgesie sollten nicht als Monotherapeutika zur Sedierung in der Endoskopie eingesetzt werden.

Empfehlungsgrad B: Evidenzstärke 5, Konsens.

\subsubsection{Opiate als Monotherapeutikum}

\subsection{Fentanyl}

\subsection{Allgemeines}

Fentanyl ist ein lipophiles, chemisch dem Pethidin verwandtes, synthetisches Morphinderivat, das ca. 600-mal potenter als Pethidin und 100-mal potenter als Morphium ist. Nach intravenöser Applikation setzt die Wirkung durch Bindung an spezifischen Opioidrezeptoren im Gehirn und Rückenmark bereits nach ca. $20 \mathrm{~s}$ ein. Das Wirkungsmaximum ist nach $6 \mathrm{~min}$ zu erwarten, wobei die Dauer der Analgesie 20-30 min be-

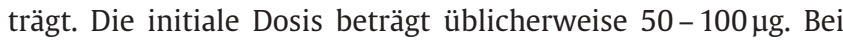
älteren Patienten ist eine entsprechende Dosisreduktion vorzunehmen. Die häufigste unerwünschte Nebenwirkung ist die Atemdepression, mit der aufgrund der starken Potenz des Präparats bereits ab einer Dosierung von $0,1 \mathrm{mg}$ (beim Erwachsenen) zu rechnen ist. Darüber hinaus kann es zu einer Thoraxrigidität kommen, welche eine etwaig notwendige Beatmung des Patienten erschweren kann. Der Effekt auf Blutdruck und Herzfrequenz sind eher gering und meist durch einen Abfall dieser Parameter aufgrund einer vagalen Stimulation bedingt. Darüber hinaus können Krämpfe der glatten Muskulatur an den Gallenwegen und Pankreas wie auch eine spastische Obstipation auftreten. Das Auftreten von Übelkeit und Erbrechen unter Fentanyl ist vergleichbar mit anderen Opiaten. Auch wenn Fentanyl üblicherweise als Anästhetikum im Rahmen der Allgemeinanästhesie (häufig in Kombination mit anderen Präparaten) oder bei chronischen Schmerzen i.A. transdermal verwendet wird, gibt es wenige Studien mit geringer Fallzahl, die die Anwendung als Analgetikum im Rahmen der Endoskopie untersucht haben.

\section{Anmerkungen}

In einer prospektiven Studie von Basu et al. [125] an 109 konsekutiven Patienten zur flexiblen Sigmoidoskopie, erhielten die Patienten auf Wunsch eine Analgesie mit einem Einzelbolus Fentanyl $(50 \mu \mathrm{g})$ oder aber keine Analgesie. Von den 46 Patienten, die sich für eine Analgesie entschieden hatten, klagten signifikant weniger über moderate/starke Schmerzen als von den 63 Patienten ohne Analgesie (9 vs. 26, p<0,05). Die Patientenzufriedenheit, insbesondere unter dem Aspekt für eine Wiederholungsuntersuchung mit dem jeweiligen Verfahren, war unter Analgesie signifikant höher als ohne Analgesie $(p<0,01)$. Ein vermehrtes Auftreten von kardiorespiratorischen Komplikationen unter Fentanylgabe wurde nicht beschrieben.

In einer weiteren randomisierten Studie von Ishido et al. [126], in der 84 Patienten zur ÖGD entweder eine Sedierung mit Fentanyl oder keine Sedierung erhalten hatten, konnte gezeigt werden, dass durch Analgesie neben einer gesteigerten Patiententoleranz auch der - durch die Endoskopie hervorgerufene - 
Anstieg der Herzfrequenz vermindert werden konnte. Neben einem minimalen Abfall des arteriellen Sauerstoffgehalts zeigte sich für die $\mathrm{O}_{2}$-Sättigung kein signifikanter Unterschied in beiden Gruppen.

\subsection{Remifentanil}

\subsection{Allgemeines}

Für die routinemäßige Verwendung von Remifentanil, einem hoch potenten synthetisch hergestellten Opioid mit extrem kurzer Halbwertszeit (2 - 3 min), gibt es derzeit nur spärliche Daten. Beachtenswert ist ein gehäuftes Auftreten von Atemdepressionen und dass die Substanz für die Analgesie bei spontan atmenden Patienten nur als kontinuierliche Infusion appliziert werden soll. Der Einsatz wird auf eine Umgebung, die vollständig zur Überwachung und Unterstützung der Atmungs- und Herz-Kreislauf-Funktionen ausgestattet ist, beschränkt (laut Fachinformation).

\section{Anmerkungen}

In einer randomisierten Studie von Akcaboy et al. [127] erhielten 100 Patienten im Rahmen der Koloskopie eine kontinuierliche Infusion von Remifentanil (Bolus: $0,5 \mu \mathrm{g} / \mathrm{kg}$, dann $0,05 \mu \mathrm{g} / \mathrm{kg} /$ min kontinuierlich) oder Propofol (Bolus: $0,5 \mathrm{mg} / \mathrm{kg}$ gefolgt von $50 \mu \mathrm{g} / \mathrm{kg} / \mathrm{min}$ ). Bei Verwendung von Remifentanil war die Untersuchungsdauer länger und die Sauerstoffsättigung nach Bolusgabe niedriger als nach Propofol. Wenngleich die Aufwachzeit nach Remifentanilgabe kürzer war, zeigte sich kein Vorteil in der Entlassungszeit. Übelkeit und Erbrechen wurden in der Remifentanilgruppe darüber hinaus wesentlich häufiger beobachtet.

\subsubsection{Ketamin als Monotherapeutikum}

2.2.4.3.1. Allgemeines

Ketamin ist ein intravenös und intramuskulär injizierbares Allgemeinanästhetikum mit starker analgetischer Wirkung, welches als Monoanästhetikum vorwiegend bei kurz dauernden diagnostischen und therapeutischen Eingriffen im Kindesalter und für spezielle Situationen im Erwachsenenalter zur Anwendung kommt. Ketamin hat nach i.v. Bolusgabe einen raschen Wirkungseintritt $(<1 \mathrm{~min})$, mit einer Wirkdauer von ca. $10-15$ min [128]. Es bewirkt eine sogenannte dissoziative Anästhesie, die mutmaßlich durch eine funktionelle Entkopplung des thalamoneokortikalen vom limbischen System hervorgerufen ist. Die analgetische Wirkung tritt bereits bei subdissoziativen Dosen auf und überdauert die Anästhesie. Die sedativen und hypnotischen Eigenschaften von Ketamin sind dagegen weit weniger ausgeprägt. Der Muskeltonus ist unter Ketamin-Anästhesie erhalten oder gesteigert, sodass die Schutzreflexe im Allgemeinen nicht beeinträchtigt werden. Die Krampfschwelle wird nicht gesenkt. Unter Spontanatmung tritt eine Erhöhung des intrakraniellen Druckes ein. Aufgrund einer sympathikotonen Wirkung führt Ketamin zu einem Anstieg von Blutdruck und Herzfrequenz, wodurch auch der myokardiale Sauerstoffverbrauch bei gleichzeitig gesteigerter Koronardurchblutung zunimmt. Am Herzen selbst zeigt Ketamin eine negativ inotrope und antiarrhythmische Wirkung. Der periphere Widerstand ändert sich aufgrund gegensätzlicher Einflüsse kaum. Nach Ketamingabe wird eine mäßige Hyperventilation beobachtet. An der Bronchialmuskulatur übt Ketamin einen relaxierenden Effekt aus. Kontraindikationen für die Anwendung sind u.a. eine schlecht eingestellte oder nicht behandelte arterielle Hypertonie (systolischer/diastoli- scher Blutdruck über 180/100 mmHg in Ruhe); Patienten, bei denen eine Blutdrucksteigerung eine erhebliche Gefährdung bedeutet (z. B. vorangegangener zerebrovaskulärer Insult).

Relative Kontraindikationen bestehen u.a. bei instabiler Angina pectoris oder Myokardinfarkt in den letzten 6 Monaten. Da die pharyngealen Reflexe im Allgemeinen erhalten sind, sollte ohne zusätzliche Verwendung von Muskelrelaxanzien eine mechanische Reizung des Pharynx vermieden werden, wenn Ketamin als Monoanästhetikum verwendet wird. Bei Eingriffen an Pharynx, Larynx und Bronchialbaum kann daher insbesondere eine Muskelrelaxation mit entsprechender Beatmung erforderlich sein. Eine unerwünschte Nebenwirkung ist das gehäufte Auftreten von Halluzinationen, Alpträumen und deliranten Zuständen, welche in 10-30\% der Fälle beobachtet wird. Durch die zusätzliche Gabe von Midazolam können diese Reaktionen vermindert werden [129].

\section{Anmerkungen}

Von einer Monotherapie mit Ketamin zum routinemäßigen Gebrauch im Rahmen endoskopischer Untersuchungen sollte aufgrund der o.g. Aspekte abgesehen werden.

Die meisten Studien zur Anwendung von Ketamin liegen im Rahmen einer Kombinationstherapie vorzugsweise - mit Midazolam - für endoskopische Eingriffe bei Kindern vor [130, 131]. Hier konnte eine adäquaten Sedierung ohne wesentliche kardiorespiratorische Komplikationen erzielt werden.

Wenngleich die Daten zur Anwendung von Ketamin bei Erwachsenen gering sind, so gibt es doch auch hier Hinweise, dass die zusätzliche Gabe von Ketamin, allerdings wiederum in Kombination mit Midazolam bei einem entsprechenden Patientengut (unter Berücksichtigung der Kontraindikationen) einen Vorteil bieten kann. In einer doppelblinden, placebokontrollierten Studie von Rosing et al. [132] zum Vergleich von Midazolam/Placebo vs. Midazolam/Ketamin bei 129 Patienten zur Koloskopie zeigte sich unter Verwendung der Kombinationstherapie neben einer geringeren Notwendigkeit für eine Nachinjektion (40 vs. 27\%), eine bessere Sedierung und Analgesie sowie eine höhere Patientenakzeptanz für eine Re-Endoskopie.

In einer randomisierten Studie von Ong et al. [133], die im Rahmen der ERCP eine Ketamin-Kombinationssedierung (Ketamin plus Midazolam, Pentazocin, Propofol) mit einer alleinigen Propofolsedierung verglichen hatte, zeigte sich gerade bei jüngeren Patienten der Vorteil des Kombinationsregimes in einer besseren Patiententoleranz. Allerdings war die Patientenzufriedenheit in beiden Gruppen nicht unterschiedlich. Darüber hinaus traten unter Verwendung der Kombinationstherapie vermehrt Hypoxien auf.

\subsubsection{Inhalationsanästhetika als Monotherapeutikum \\ 2.2.4.4.1. Distickstoffmonoxid (Lachgas)}

\subsection{Allgemeines}

Medizinisches Lachgas $\mathrm{N}_{2} \mathrm{O}$ ist ein stabiles, reaktionsträges, farb- und geruchloses Gas, das stark analgetisch und schwach narkotisch wirkt. Die Willkürmotorik bleibt unbeeinflusst. Der Vorteil liegt in einem raschen Wirkungseintritt und einer schnellen Erholungszeit. Im Gemisch mit Sauerstoff wird es üblicherweise zur Anästhesie-Einleitung, im Rahmen einer Kombinationsnarkose oder zur Analgesie unter stationären Bedingungen in der klinischen Geburtshilfe eingesetzt. Medizinisches Lachgas $\mathrm{N}_{2} \mathrm{O}$ ist zur inhalativen Anwendung bestimmt. Es darf grundsätzlich erst nach Verdampfung (Überführung in den Gaszustand) und mithilfe von geeigneten Inhalationsgerä- 
ten bzw. Narkoseapparaten verabreicht werden. Obwohl nur eine geringe Wirkung auf den Kreislauf zu erwarten ist, kann es zur Senkung des Blutdrucks, zur Abnahme des Schlagvolumens und zur Steigerung des pulmonalvaskulären Widerstands kommen. Ebenso kann es zu einer Dilatation der Hirngefäße mit nachfolgender Steigerung des intrakraniellen Druckes führen. An unerwünschten Nebenwirkungen können Übelkeit und Erbrechen auftreten. Das Auftreten von Euphorien, Träumen und Fantasien wird beschrieben. Bei Überdosierung können Hypoxie, Kreislaufdepression, Agitation oder Somnolenz bis Bewusstlosigkeit auftreten.

\section{Anmerkungen}

In einer randomisierten Studie von Saunders et al. [134] zum Vergleich der patientenkontrollierten Lachgasinhalation versus intravenöser Midazolam/Pethidin Gabe im Rahmen der Koloskopie zeigte sich neben einer signifikant schnelleren Aufwachzeit nach Lachgasinhalation ( 32 vs. $60 \mathrm{~min}, \mathrm{p}=0,01$ ), ein geringerer Abfall des Blutdrucks und der Sauerstoffsättigung. Allerdings traten unter Lachgasinhalation gehäuft Kopfschmerzen auf.

In einer weiteren Studie von Forbes et al. [135] erhielten Patienten zur Koloskopie randomisiert entweder Lachgas oder Midazolam/Meperidin (mittlere Dosierung: 4,7 mg und $55 \mathrm{mg}$ ). Lachgas war weniger effektiv, die Patienten erinnerten sich häufiger an Schmerzen während der Untersuchung $(\mathrm{p}<0,001)$ und waren signifikant unzufriedener mit der Untersuchung ( $p<0,01$ ), hingegen war die Aufwachzeit signifikant schneller als unter Midazolam/Meperidin (30 vs. $60 \mathrm{~min}, \mathrm{p}=0,0001$ ). In der kürzlich als Abstrakt veröffentlichten Studie von Maleskar et al. [136], in der 100 Patienten randomisiert entweder Lachgas oder Midazolam/Fentanyl im Rahmen der Koloskopie erhielten, war im Gegensatz zu der Studie von Forbes die Patientenzufriedenheit unter Lachgas besser, die Patienten gaben weniger Schmerzen an und die Zeit bis zur Entlassung war signifikant kürzer ( 26 vs. 44 min, $p=0,004$ ).

\subsubsection{Nicht steroidale Antiphlogistika}

2.2.4.5.1. Keterolac trometamol

\subsection{Allgemeines}

Ketorolac trometamol ist ein nicht steroidaler Entzündungshemmer mit analgetischer Wirkung und raschem Wirkungseintritt von wenigen Minuten nach i.v. Injektion, dessen Wirkungsmechanismus im Wesentlichen in der Hemmung der Prostaglandin-Biosynthese beruht. Üblicherweise wird es zur Behandlung von akuten Schmerzzuständen verwendet. In Deutschland ist die Substanz nur zur Applikation in Augentropfen erhältlich.

Ebenso wie andere nicht steroidale Entzündungshemmer kann Keterolac trometamol gastrointestinale Reizungen, Geschwüre, Blutungen oder Perforationen mit oder ohne vorausgehenden Symptomen verursachen; es sollte daher insbesondere bei Patienten mit bekannter Neigung zu Krankheiten des Gastrointestinaltrakts nicht verabreicht werden. Weitere unerwünschte Nebenwirkungen sind Flüssigkeitsretention und Ödeme, weshalb Vorsicht bei Patienten mit Herzdekompensation und Hypertension geboten ist. Auch treten gehäuft Kopfschmerzen, Benommenheit, Schläfrigkeit, Schwitzen und Schwindel auf.

\section{Anmerkungen}

In einer randomisierten, doppelblinden, placebokontrollierten Studie von Mui et al. [137] wurde der Effekt von intravenös verabreichtem Ketorolac trometamol $(60 \mathrm{mg})$ als Prämedikation 30 min vor Beginn einer patientenkontrollierten Sedierung (mittels Propofol/Alfentanil) zur Koloskopie an insgesamt 140 Patienten untersucht.

Sowohl die von den Patienten als auch von den Endoskopikern bewerteten Schmerz-Scores waren unter Prämedikation mit Ketorolac signifikant niedriger als in der Kontrollgruppe (3,99 vs. $5,28, p=0,006$ und 80,0 vs. $57,1 \%, p=0,004)$. Hinsichtlich der benötigten Medikamenten-Dosis, Patientenzufriedenheit und hämodynamischen Veränderungen ergab sich in beiden Gruppen kein signifikanter Unterschied.

Weitere Daten, welche die tatsächliche Überlegenheit von NSRA gegenüber den derzeit gebräuchlichen Sedierungsregimen belegen, fehlen, sodass hier keine generelle Empfehlung für den routinemäßigen Gebrauch von NSAR gegeben werden kann.

\subsection{Kombinationstherapien}

\subsubsection{Allgemeines}

Kombinationstherapien beinhalten in der Regel die Kombination eines Sedativums mit einem Analgetikum oder die Kombination verschiedener Sedativa. Während die alleinige Sedierung mit Benzodiazepinen, wie Diazepam oder Midazolam [138-141], die Komplikationsrate bezogen auf stressinduzierte kardiovaskuläre Komplikationen (z.B. Myokardischämien, Erhöhung des Rate-Pressure-Products) senkt, führt darüber hinaus die Kombination mit einem Opiat [142-144] oder Hypnotikum wie Propofol $[70,145]$ zu einer höheren Patientenakzeptanz.

Auch kann durch Kombination verschiedener Substanzen neben einem Einspareffekt in der benötigten Dosis [146] eine schnellere Aufwachzeit erzielt werden [59, 57, 143, 146]. Allerdings gilt es zu berücksichtigen, dass es unter einer Kombinationstherapie im Vergleich zu einer Monotherapie gehäuft zu einem Abfall von Blutdruck und Sauerstoffsättigung kommen kann [147-149] (siehe auch Abschnitt 2.3.3.).

\subsubsection{Spezifische Kombinationen}

\subsubsection{Kombination Sedativum plus Opiat}

Empfehlungen

Die Kombination eines Sedativums mit einem Analgetikum führt zu einer höheren Patientenakzeptanz bei geringerem Sedativabedarf. Bei entsprechenden strukturellen und personellen Voraussetzungen sowie schmerzhaften Eingriffen kann eine Kombinationstherapie mit einem Opiat sinnvoll sein (zu den entsprechenden Voraussetzungen Verweis auf AG Strukturqualität). Empfehlungsgrad: 0, Evidenzstärke 1b, Konsens.

\section{Anmerkungen}

In einer Studie von Milligan et al. [143] zeigte die Kombination von Alfentanil/Midazolam im Vergleich zur alleinigen Gabe von Midazolam bei oberer Intestinoskopie, neben einer Verbesserung der Untersuchungsbedingungen für den Endoskopiker, eine höhere Patientenakzeptanz und eine schnellere Aufwachzeit.

In einer weiteren randomisierten, doppelt geblindeten Studie von Radaelli et al. [144] zum Vergleich von Midazolm versus Midazolam/Meperidin im Rahmen der Koloskopie bei 253 Patienten, wurden von den Patienten unter Kombinationstherapie signifikant weniger Schmerzen und einer höhere Bereitschaft 
für eine Wiederholungsuntersuchung angegeben. Die Aufwachzeit und ein Abfall der Sauerstoffsättigung waren in beiden Untersuchungsgruppen nicht unterschiedlich.

In einer randomisierten, kontrollierten Studie von VanNatta et al. [150] erhielten 200 Patienten zur Koloskopie entweder nur Propofol zur tiefen Sedierung oder eine Kombinationstherapie mit Propofol/Fentanyl, Propofol/Midazolam oder Propofol/Midazolam/Fentanyl, um eine moderate Sedierung zu erzielen. Verglichen wurden Aufwachzeit, Patientenzufriedenheit und Vitalparameter. Patienten unter alleiniger Propofolsedierung benötigten signifikant höhere Dosierungen und zeigten signifikant tiefere Sedierungsstadien im Vergleich mit den anderen Kombinationstherapien $(\mathrm{p}<0,001)$. Die Entlassungszeit nach Kombinationstherapie war schneller als unter alleiniger Propofolgabe (Median 13,0 - 14,7 versus 18,1 min, $\mathrm{p}<0,01$ ). Hinsichtlich der Vitalparameter zeigte sich kein Unterschied zwischen den einzelnen Untersuchungsgruppen, auch die Patientenzufriedenheit war nicht signifikant unterschiedlich.

\subsubsection{Kombination Sedativum plus Propofol}

Empfehlung

Die Kombination unterschiedlicher Sedativa (z.B. Midazolam plus Propofol) führt zu gleicher Akzeptanz/Toleranz bei vermutlich geringerer Propofol-Dosis. Bei entsprechender struktureller und persönlicher Voraussetzung kann eine Kombinationstherapie Midazolam/Propofol sinnvoll sein.

Empfehlungsgrad: B, Evidenzstärke 1b, starker Konsens.

\section{Anmerkung}

In einer Studie von Cordruwisch et al. [151] an 64 Patienten, die im Rahmen zweier aufeinanderfolgender, länger dauernder (>30 min) endoskopischen Untersuchungen zunächst mit Propofol und nachfolgend mit der Kombination Midazolam/Propofol sediert wurde, lag der Vorteil der Kombinationssedierung in einem deutlichen Einspareffekt von Propofol (der mit 59\% angegeben wurde). Die postinterventionelle Aufwachzeit war unter dem Kombinationsregime allerdings doppelt so lang ( 4 vs. $8 \mathrm{~min}$ ).

Eine Studie von Seifert et al. [146] an 239 konsekutiven Patienten zur therapeutischen Endoskopie (ÖGD und EUS) ergab ebenfalls, dass die Kombination von Propofol mit Midazolam zu einer Dosiseinsparung von Propofol im Vergleich zur alleinigen Propofolgabe $(0,20 \pm 0,09 \mathrm{mg} / \mathrm{min} / \mathrm{kg}$ vs. $0,25 \pm 0,13 \mathrm{mg} / \mathrm{min} / \mathrm{kg}$, $\mathrm{p}<0,01$ ), bei ansonsten gleicher Effektivität führt. Das Kombinationsregime war allerdings auch hier mit einer verlängerten postinterventionellen Erholungszeit verbunden $(25 \pm 8 \mathrm{~min}$ vs. $19 \pm 7, \mathrm{p}<0,05$ ). Ähnliche Ergebnisse hinsichtlich des Einspareffekts bei Kombination von Propofol und Midazolam lieferte die bereits oben zitierte Studie von VanNatta et al. [150]. Hier waren jedoch die postinterventionelle Erholungszeiten in den Armen mit Kombinationstherapie kürzer als bei alleiniger Propofolgabe $(13,0-14,7$ versus $18,1 \mathrm{~min}, \mathrm{p}<0,01)$.

\subsubsection{Kombination Sedativum plus Spasmolytikum}

Empfehlung

Die Kombination von Spasmolytika und Sedativa erhöht die Rate von kardiovaskulären Nebenwirkungen und reduziert sowohl die Patientenzufriedenheit als auch die Untersucherbeurteilung, daher sollte der Einsatz von Spamolytika bei der Endoskopie in Sedierung sorgfältig erwogen werden.

Empfehlungsgrad: A, Evidenzstärke 1b, starker Konsens.

\section{Anmerkung}

In einer prospektiven, doppelt verblindeten, placebokontrollierten Studie von Mui et al. [152] wurde die Verwendung des Spasmolytikums Hyoscin N-Butylpromide (Buscopan) zur Prämedikation bei patientenkontrollierter Sedierung mittels Propofol/Alfentanil im Rahmen der Koloskopie untersucht. Hier zeigte sich neben einer verlängerten Caecum-Intubations-Zeit und signifikant geringeren Untersucherzufriedenheit auch ein signifikant erhöhter Bedarf an Sedativum/Analgetikum sowie eine signifikante hämodynamische Instabilität.

\subsubsection{Nebenwirkungen bei Kombinationstherapie}

\subsubsection{Allgemeines}

Trotz Einhaltung der empfohlen Dosisreduktion im Rahmen einer Kombinationstherapie kommt es häufiger zu einer Kompromittierung der respiratorischen Funktionen als unter Monotherapie [147-149]. Auf Basis der Studienlage kann nicht gezeigt werden, ob lebensbedrohliche Situationen unter Kombinationstherapie häufiger auftreten.

\section{Anmerkungen}

In einer Studie von Rembacken et al. [149] zum Stellenwert von Pethidin bei der Koloskopie erhielten 71 Patienten in einer randomisierten, doppelt verblindeten, placebokontrollierten Studie eine Sedierung mit nur Diazepam oder Diazepam in Kombination mit Pethidin. Während die Patientenzufriedenheit in beiden Gruppen vergleichbar war, zogen die Untersucher die Kombinationstherapie aufgrund einer besseren Patiententoleranz vor. Allerdings kam es unter Kombinationstherapie doppelt so häufig zu einem Abfall der Sauerstoffsättigung als unter alleiniger Gabe von Diazepam $(p=0,008)$.

In einer weiteren Studie von DiPalma et al. [147] zum Stellenwert von Alfentanil im Rahmen der Koloskopie erhielten 35 Patienten randomisiert entweder eine Kombinationstherapie mit Alfentanil/Midazolam oder Midazolam alleine. Auch hier zeigte sich bei Kombinationstherapie ein gehäufter Abfall der Sauerstoffsättigung mit der Notwendigkeit der Sauerstoffgabe. Patiententoleranz, Patientenzufriedenheit, Aufwachzeit und Blutdruck waren in beiden Gruppen nicht unterschiedlich.

In einer randomisierten, doppelt geblindeten Studie von Moerman et al. [148] wurde die additive Gabe Remifentanil zur Sedierung mittels Propofol bei 50 relativ gesunden Patienten (ASA I und II) zur Koloskopie untersucht. In der Remifentanil/PropofolGruppe kam es signifikant häufiger zu einem Abfall von Blutdruck und Sauerstoffsättigung. Und obwohl die Gabe von Remifentanil zu einer Dosisreduktion von Propofol führte, war die Aufwachzeit unter alleiniger Propofolsedierung signifikant kürzer $(p<0,01)$ und die Patientenzufriedenheit signifikant höher $(\mathrm{p}<0,01)$.

\subsubsection{Monitoring/Strukturqualität Empfehlung}

Aufgrund arzneimittelbedingter Interaktionen soll bei Kombinationstherapien das jeweilig benutzte Medikament bzgl. der Dosis angepasst werden. Zudem muss bei dem schwer zu kalkulierenden Risiko besonders auf die Verfügbarkeit eines adäquaten Monitorings und die unverzügliche Interventionsmöglichkeit durch einen weiteren, in der kardiopulmonalen Reanimation und Beatmung (einschließlich Intubation und manuelle Beatmung) erfahrenen Arztes geachtet werden (s.a. Strukturqualität). Empfehlungsgrad: A, Evidenzstärke 1b, Konsens. 


\subsection{Einfluss der Ko-Morbidität}

\subsubsection{Allgemeines}

Komorbidität beinhaltet im Wesentlichen eine höhere ASAKlassifikation sowie Begleiterkrankungen (auch bei ASA II und III), welche mit einer höheren Nebenwirkungsrate verbunden sind $[71,153,154]$.

Gerade ältere Patienten und insbesondere Patienten mit bereits zugrunde liegenden kardialen oder pulmonalen Erkrankungen haben ein höheres Risiko für das Auftreten von Komplikationen im Rahmen einer Endoskopie mit Sedierung [71, 138, 155]. Aber auch hepatobiliäre Erkrankungen mit verminderter Elimination von Pharmaka oder ein altersbedingt verlangsamter Metabolismus können zu einer Potenzierung der erwähnten Nebenwirkungen führen $[156,157]$.

Eine Vielfalt an physiologischen Prozessen trägt hier zu einer erhöhten Sensitivität gegenüber den verschiedenen Substanzen mit einem entsprechend erhöhtem Sedierungsrisiko bei [158]. So verschlechtert sich mit zunehmendem Alter die arterielle Sauerstoffsättigung, mit oder ohne zusätzliche Sauerstoffgabe. Auch die kardiorespiratorische Reaktion auf Hypoxie oder Hyperkapnie ist vermindert und verzögert. Narkotika und andere zentral wirksame Substanzen führen zu einer verstärkten Atemdepression und gehäuftem Auftreten einer transienten Apnoe. Altersbedingte Erkrankungen und schnelle oder übermäßige Dosierungen tragen hier mehr für das Auftreten von kardiorespiratorischen Komplikationen bei als das Alter per se [158].

\subsubsection{Risikopatienten}

Empfehlung

Patienten mit höherer ASA-Klasse und/oder ältere Patienten weisen ein höheres Risiko sedierungsbedingter Nebenwirkungen auf (kardiorespiratorische Depression). Die Dosis des benutzten Sedativums sollte dementsprechend angepasst bzw. verringert werden. Empfehlungsgrad: B, Evidenzstärke 2b, starker Konsens.

\subsubsection{Substanzart}

\section{Empfehlung}

Eine Sedierung mit Propofol kann auch bei älteren Patienten, $\mathrm{Pa}$ tienten mit kardiorespiratorischen Erkrankungen sowie bei Lebererkrankungen als Ersatz für die Verwendung von Benzodiazepien erwogen werden. Theoretisch ist z.B. eine Verschlechterung der hepatischen Enzephalopathie, welche für die Benzodiazepine belegt ist, durch Propofol nicht zu erwarten.

Empfehlungsgrad: 0, Evidenzstärke 2b, Konsens.

\section{Anmerkungen}

Entsprechend der Modifikation der Empfehlungen der American Society of Gastroenterology für ältere Patienten im Rahmen der gastrointestinalen Endoskopie [159] sollten bei diesen Patienten weniger Substanzen langsamer appliziert und Substanzen mit geringer Kumulationsdosis [159, 160] verabreicht werden.

Wie bei jungen Patienten werden zur Sedierung von älteren Patienten meist Midazolam und/oder andere Opioide/Narkotika verwendet. Da bei der Verwendung von Benzodiazepinen ein erhöhtes Hypoxämie-Risiko bei älteren Patienten, Patienten mit Übergewicht und anämischen Patienten besteht, erscheint eine Dosisminderung auch hier ratsam [161]. Für diese Substanzgruppe besteht zudem auch postinterventionell ein erhöhtes Hypoxämierisiko, insbesondere bei älteren Patienten [121, 162]. Weiterhin führen Benzodiazepine bei Patienten mit Leberzirrhose zur Verstärkung einer hepatischen Enzephalopa- thie [156, 157, 163, 164], die bei der alleinigen Verwendung von Propofol nicht beobachtet werden kann [164].

In einer randomisierten Studie von Riphaus et al. [164] bei insgesamt 60 Patienten mit bekannter Leberzirrhose und portaler Hypertension erhielten diese zur Sedierung bei der Gastroskopie in interventioneller Intention (Varizenligatur), randomisiert im Verhältnis 2:1, entweder Propofol oder Midazolam. Alle Patienten absolvierten vor und $2 \mathrm{~h}$ nach Beendigung der Untersuchung einen Zahlenverbindungstest (ZVT-A) sowie einen portosystemischen Enzephalopathie-Syndrom-Test (PSE), bestehend aus 4 zusätzlichen Einzeltest-Komponenten. Die Auswertung erfolgte anhand eines sich hieraus ergebenden Gesamtscores. Als Kontrollgruppe dienten 20 Patienten ohne Leberzirrhose, die keiner Gastroskopie unterzogen wurden. Darüber hinaus wurden die Aufwachzeit und der Aufwachscore bestimmt. Bei den mit Propofol sedierten Patienten zeigt sich eine im Vergleich zu Midazolam deutliche Verkürzung der Aufwachzeit (7,8 $\pm 2,9$ min. vs. $18,4 \pm 6,7 \mathrm{~min}$.). Darüber hinaus zeigte sich nach Sedierung mit Propofol auch ein geringerer Effekt auf den PSE-Gesamtscore im Vergleich zu Midazolam, dessen Verwendung zu einer deutlichen Aggravation einer bestehenden subklinischen, hepatischen Enzephalopathie führte. Eine Sedierung mit Propofol führt daher tendenziell nicht zur Exazerbation einer subklinischen, hepatischen Enzephalopathie bei Patienten mit Leberzirrhose und stellt somit ein alternatives Sedativum für diese Patienten dar.

Da Propofol einen engen therapeutischen Bereich hat, kann es bei älteren Hochrisikopatienten im Vergleich zu jüngeren gehäuft zu kardiorespiratorischen Komplikationen kommen [71, 165].

Niedrige Initialdosen von Propofol, im Allgemeinen die Hälfte der empfohlenen Dosis für Erwachsene, sowie eine langsame, allmähliche Titrierung und ein sorgfältiges Monitoring erscheinen daher bei Sedierung älterer Patienten sinnvoll [166, 167]. Unter Beachtung der besonderen Sorgfalt bei der Sedierung ältere Patienten konnte gezeigt werden, dass Propofol dann auch bei dieser Patientengruppe sicher angewandt werden kann [121, 165].

In einer Kohortenstudie von Vargo et al. [153] zur Frage nach Risikofaktoren für kardiopulmonale Ereignisse im Rahmen einer Propofol-Sedierung bei oberer und unterer Intestinoskopie wurde das Gesamtrisiko für das Auftreten eines kardiopulmonalen Ereignisses während 528 Gastroskopien und 1683 Koloskopien mit 11,7/1000 Fälle angegeben. Ein erhöhtes Risiko zeigte sich hier bei Patienten mit ansteigender ASA-Klassifikation im Rahmen der Koloskopie.

In einer Studie von Heuss et al [165] zur Sicherheit der Propofolsedierung bei endoskopischen Untersuchungen (Gastroskopie und Koloskopie) von Hochrisikopatienten (ASA III und IV) im Vergleich zu Patienten mit ASA-Klassifikation I und II zeigte sich ebenfalls bei den Hochriskopatienten ein erhöhtes Risiko für einen kurzfristigen, jedoch relevanten Abfall der Sauerstoffsättigung unter 90\% (3,6 versus 1,7\% für ASA I and II $[\mathrm{p}=0,036]$ ). In vier im Vergleich zu einem Fall (ASA I und II) war eine kurzfristige Maskenbeatmung notwendig. Die benötigte Dosis für eine adäquate Sedierung lag bei den Hochrisikopatienten 10-20\% unter der benötigten Dosis der Patienten mit ASA-Klassifikation I und II. Ein entsprechend sorgfältiges Monitoring bei älteren Hochrisikopatienten wird von den Autoren daher gefordert.

In einer randomisierten, kontrollierten Studien von Riphaus et al. [121] wurden 150 Patienten über 80 Jahre im Rahmen der 
ERCP entweder mittels Propofol oder Midazolam/Pethidin sediert. Klinisch relevante kardiorespiratorische Veränderungen wurden unter Propofol nicht häufiger als unter Midazolam/Pethidin beobachten. Die Aufwachzeit war unter Propofol signifikant kürzer ( $22 \pm 7$ min vs. $31 \pm 8$ min für Midazolam/Pethidin $[\mathrm{p}<0,01])$ und es kam im Rahmen der postinterventionellen Überwachung signifikant seltener zu einem Abfall der Sauerstoffsättigung unter 90\% (12 vs. 26\% für Midazolam/Pethidin, $\mathrm{p}<0,01)$.

Es gilt zu berücksichtigen, dass bei multimorbiden Patienten und solchen mit erhöhtem Risikoprofil bestimmte personelle Voraussetzungen (s.Kap. 3.3.) zu beachten sind.

\subsection{Musik in der Endoskopie \\ Empfehlung}

Die Verwendung von beruhigender Musik hat einen positiven Einfluss auf die Patientenakzeptanz bei Koloskopien und führt zu einer Verminderung der durchschnittlichen Dosis von Propofol oder Midazolam.

Empfehlungsgrad B, Evidenzstärke 2b, starker Konsens.

\section{Anmerkung}

In einer prospektiv randomisierten Studie von Harikumar et al. [166] bei koloskopierten Patienten wurde der Einfluss beruhigender Musik auf die Dosis einer patientenkontrollierten Sedierung mit Propofol untersucht [166]. Musik verminderte hier signifikant die Dosis von Propofol und von Midazolam und führte zu einer besseren Patientenakzeptanz.

In einer aktuellen Metaanalyse von Rudin et al. [167] wurden 6 randomisierte, kontrollierte Studien mit insgesamt 641 Patienten bewertet. In 3 Studien erfolgte die Endokopie unter alleiniger Musiktherapie, wodurch die Angst der Patienten im Vergleich zur Kontrollgruppe um 8,6\% reduziert werden konnten $(p=0,004)$. In den 3 verbliebenen Studien erhielten die Patienten zusätzlich zur medikamentösen Therapie (Midazolam, Pethidin oder Propofol/Alfentanil) eine Musiktherapie. Dadurch kam es zu einer signifikanten Reduktion der benötigten Analgetika um 29,7\% ( $=0,001)$ und Sedativa um 15\% $(p=0,055)$.

\section{Strukturqualität: Persönliche/personelle/apparative Voraussetzungen \\ $\nabla$}

Da zu dem Themenkomplex der Strukturqualität derzeit keine prospektiven Studien existieren, wurden für die Empfehlungen fast ausnahmslos die bereits vorhanden Leitlinien und Empfehlungen [168-186] herangezogen.

\section{Einleitung}

Sowohl der diagnostische oder therapeutische Eingriff als auch die Sedierung sind eigenständige medizinische Verfahren. Führt ein Arzt den diagnostischen oder therapeutischen Eingriff und gleichzeitig das Sedierungsverfahren durch, übernimmt er nicht nur für den Eingriff, sondern auch für die Sedierung und/oder die Analgesie einschließlich der Überwachung und gegebenenfalls Wiederherstellung vitaler Funktionen die volle Verantwortung.

Spezielle Kenntnisse in Theorie und Praxis der Sedierung und/ oder Analgesie sind nicht nur für den Arzt, sondern auch für das ihn unterstützende nicht ärztliche Personal notwendig. Ein Arzt kann nicht in Personalunion zur gleichen Zeit den invasiven Eingriff durchführen und die Sedierung und/oder das Analgesieverfahren überwachen.
Nicht der den Eingriff durchführende Arzt, sondern eine speziell geschulte, in der Regel ärztliche Person sollte verantwortlich sein für das Sedierungsverfahren und die Überwachung der Vitalfunktionen.

Die die Sedierung überwachende Person darf in dieser Zeit keine anderen Aufgaben wahrnehmen. Ob diese ärztliche Person im individuellen Fall - ausgenommen sind tiefe, durch Propofol oder Kombinationssedierung erzeugte Sedierungsgrade/Allgemeinanästhesien - durch qualifiziertes, speziell geschultes nicht ärztliches Personal ersetzt werden kann, ist im jeweiligen Einzelfall von dem die diagnostische oder therapeutische Intervention durchführenden Arzt unter Berücksichtigung der Struktur der Arbeitsstätte, des Zustands des Patienten und der Komplexität der Intervention vor Ort zu beurteilen und zu verantworten.

Der den Eingriff durchführende Arzt muss sich vergewissern, dass diese Person ausreichend qualifiziert und in der Lage ist, ihre Aufgaben adäquat zu erfüllen.

Die Problematik des Organisations-/Übernahmeverschuldens ergibt sich aus den allgemeinen Rechtsgrundsätzen, welche sich aus dem Zivil-, Straf- und Berufsrecht herleiten. Den detaillierten Hinweisen der Hersteller der jeweils verwendeten Pharmaka, insbesondere zur Strukturqualität (z.B. apparative und personelle Ausstattung), ist zu folgen.

\subsection{Persönliche Voraussetzungen \\ Empfehlung}

Der die Sedierung durchführende und für die Sedierung verantwortliche Arzt soll in der Intensivmedizin erfahren sein. Er soll in der Anwendung von Sedativa und damit in Kenntnis, Erkennung und Behandlung der zu erwartenden Nebenwirkungen, einschließlich der kardiopulmonalen Reanimation, dem Freimachen/Freihalten eines durchgängigen Luftwegs, der Intubation und manuelle Beatmung speziell geschult sein und sie beherrschen.

Empfehlungsgrad: A, Evidenzstärke 4, starker Konsens.

\section{Anmerkung}

Analog zu den Leitlinien anderer Fachgesellschaften [10, 11, $21,22,35,37,41,166,187]$ zählen zu den persönlichen Voraussetzungen für die Durchführung einer Analgosedierung die Beherrschung der Notfallsituation mit Korrektur Kreislaufzirkulatorischer Probleme und die Fähigkeit zur endotrachealen Intubation.

\subsection{Ausbildungs- und Trainingskurse \\ Empfehlung}

Im Rahmen der Qualitässicherung soll ein speziell auf die Sedierung ausgerichtetes Training für Ärzte und nicht ärztliches Assistenzpersonal durchgeführt werden.

Empfehlungsgrad: A, Evidenzstärke 4, starker Konsens.

\section{Anmerkungen}

Spezielle, auf die Prämedikation und das Notfallmanagement ausgerichtete Trainingsvorschriften existieren bisher nur vereinzelt. Sie zeigen, dass ein spezifisches Training, auch in Form von Simulationskursen, zur verbesserten Handlungssicherheit von Ärzten führt [188].

Ein weitaus umfangreicheres Trainingsprogramm für nicht ärztliches Assistenzpersonal war Gegenstand spezieller Studien mit Propofol $[14,73]$. 


\subsection{Personelle Voraussetzungen}

\subsubsection{Ausbildungsvoraussetzungen}

Empfehlung

Die Qualifikation des ärztlichen sowie des nicht ärztlichen Personals, welches an der Durchführung der Sedierung, Überwachung und Nachsorge beteiligt ist, soll durch periodische Teilnahme an strukturierten Fortbildungscurricula sichergestellt werden. Neben theoretischen Kenntnissen werden auch praktische Fähigkeiten inkl. Komplikationsmanagement (z.B. im Simulatormodell) in diesen Curricula vermittelt. Hierbei sollte insgesamt ein gemeinsames Training des gesamten Untersucherteams (ärztliches und nicht ärztliches Personal) bevorzugt werden.

Empfehlungsgrad: A, Evidenzstärke 5, starker Konsens.

\subsection{2. Überwachung der Sedierung}

\section{Empfehlung}

Der endoskopierende Arzt ist während der Durchführung der Endoskopie in aller Regel nicht in der Lage, den Vitalfunktionen des Patienten die notwendige Aufmerksamkeit zu schenken. Es ist daher für jede Endoskopie unter Sedierung erforderlich, dass neben dem endoskopierenden Arzt und seiner Endoskopieassistenz eine weitere Person, die nicht in die Endoskopie involviert ist, diese Aufgabe zuverlässig wahrnimmt. Diese qualifizierte Person soll in der Überwachung von Patienten, die Sedativa, Hypnotika und/ oder Analgetika erhalten, speziell und nachweislich geschult und erfahren sein. Wann immer der Patient ein erhöhtes Risiko aufweist oder ein langwieriger und aufwendiger Eingriff zu erwarten ist, soll ein zweiter, entsprechend qualifizierter Arzt zugegen sein, der ausschließlich die Durchführung und Überwachung der Sedierung sicherstellt.

Empfehlungsgrad: A, Evidenzstärke 5, starker Konsens.

\section{Anmerkung}

In den Leitlinien der DGAI [10] heißt es: „Da der Untersucher während der Durchführung der Endoskopie in aller Regel nicht in der Lage ist, den Vitalfunktionen des Patienten die notwendige Aufmerksamkeit zu schenken, ist es erforderlich, dass eine zweite, in der Patientenüberwachung speziell geschulte, qualifizierte Person diese Aufgabe zuverlässig wahrnimmt“.

\section{Empfehlung}

Die Überwachungsmaßnahmen sollen sich nach dem Gesundheitszustand des Patienten, der Invasivität des durchzuführenden endoskopischen Eingriffs und der Art der Sedierung richten.

Empfehlungsgrad: A, Evidenzstärke 5, starker Konsens.

\subsubsection{Durchführung der Sedierung}

3.3.3.1. Voraussetzungen bei durch Pflegepersonal verabreichter Propofol-Sedierung (sog. „Nurse-administered propofol sedation [NAPS]“")

Empfehlung

Bei einfachen endoskopischen Untersuchungen und risikoarmen Patienten soll die Sedierung durch den entsprechend qualifizierten
Arzt eingeleitet werden und kann anschließend von einer entsprechend ausgebildeten und erfahrenen Person überwacht werden. Die die Sedierung überwachende Person darf in dieser Zeit keine anderen Aufgaben wahrnehmen. Propofol kann von einer entsprechend ausgebildeten und erfahrenen Person, die ausschließlich mit dieser Aufgabe betraut ist, auf ärztliche Anordnung während der Untersuchung verabreicht werden.

Empfehlungsgrad: A, Evidenzstärke 1b, starker Konsens.

\section{Anmerkungen}

Unter entsprechenden Voraussetzungen ist NAPS sicher und effizient [14-17, 73] bei Patienten mit ASA-Klassifikation I-III ( $\odot$ Tab. 12). Bei ASA-IV/V-Patienten soll keine NAPS erfolgen $[14-17,73]$. NAPS sollte von einer entsprechend ausgebildeten Person, die ausschließlich mit dieser Aufgabe betreut ist, durchgeführt werden.

\subsubsection{Einschränkungen für die durch Pflegepersonal verabreichte Propofol-Sedierung („NAPS“) \\ Empfehlung}

Bei Patienten mit erhöhtem Risikoprofil (siehe Risikoabschätzung 1.4.) oder komplexen, lang dauernden therapeutischen Eingriffen, die einer tieferen Sedierung bedürfen und dadurch mit einem erhöhten Sedierungsrisiko assoziiert sein können, soll ein zweiter, intensivmedizinisch erfahrener, Arzt die Sedierung durchführen. Empfehlungsgrad: A, Evidenzstärke 5, starker Konsens.

\subsection{4. Überwachung nach der Endoskopie \\ Empfehlung}

Nach Beendigung der Untersuchung sollen die Patienten bezüglich der Sedierung überwacht werden.

Empfehlungsgrad: A, Evidenzstärke 5, starker Konsens.

\section{Anmerkung}

Eine postinterventionelle Überwachung ist notwendig, um mögliche Folgen der Sedierung zu erkennen. Die Dauer der postinterventionellen Überwachungsphase ist von dem zu erwartenden Risiko abhängig [20]. Die Wirkdauer und Halbwertszeit der verwendetet Substanz ist hierbei zu berücksichtigen.

Die lückenlose Überwachung der Patienten durch qualifiziertes Personal unabhängig von der verwendeten Substanz, ggf. unter Verwendung eines Pulsoxymeters, ist fortzusetzen, bis sich der Patient vollständig erholt hat. Patienten können aus dem Überwachungsbereich entlassen werden, wenn die Vitalzeichen stabil sind und sie orientiert sind [10].

\section{Empfehlung}

Die Überwachung des Patienten in der Aufwachphase soll durch entsprechend geschultes und qualifiziertes Personal vorgenommen werden.

Empfehlungsgrad: A, Evidenzstärke 5, starker Konsens.

Tab.12 Beobachtete kardiorespiratorische Komplikationsraten bei „NAPS“-Prozeduren (n. u. = nicht untersucht).

\begin{tabular}{|lllll} 
Autor & $\mathbf{n}$ & Prozeduren & assistierte Ventilation & Hypotension (RR syst. $<90$ mmHg) \\
\hline Rex DK et al. [16] & 2.000 & ÖGD und Koloskopie & $0,2 \%$ & $0 \%$ \\
\hline Heuss LT et al. [14] & 2.547 & ÖGD und Koloskopie & $0,002 \%$ & $0,08 \%$ \\
\hline Sieg A et al. [189] & 3.641 & ÖGD und Koloskopie & $0,14 \%$ & $0,3 \%$ \\
\hline Rex DK et al. [15] & 36.743 & ÖGD und Koloskopie & $0,2 \%$ & n.u. \\
\hline Tohda G et al. [18] & 27.500 & ÖGD und Koloskopie & $0 \%$ & $2,0 \%$ \\
\hline
\end{tabular}




\section{Anmerkung}

Die entsprechende Person muss ständig im Aufwachbereich sein bzw. die Überwachungseinheit im Blickkontakt haben. Sie darf jedoch z.B. telefonieren oder Befunde abheften. Aus Gründen der Patientensicherheit sollen auch bereits wache Patienten bis zur Entlassung im Überwachungsbereich verweilen. Somit kann verhindert werden, dass der evtl. noch beeinträchtigte Patient eigenständig den Untersuchungsbereich verlässt (s. BGH-Urteil, Aktenzeichen: VI ZR265/02).

\subsection{Räumliche Ausstattung Empfehlung}

Die Sedierung soll nur in einer Umgebung durchgeführt werden, die hinreichend zur Überwachung und Unterstützung der Atmungs- und Herz-Kreislauf-Überwachung ausgestattet ist. Ein zusätzlicher und separater Aufwachbereich soll vorgehalten werden.

Empfehlungsgrad: A, Evidenzstärke 5, starker Konsens.

\section{Anmerkung}

Entsprechend den Leitlinien der DGAI [10] „müssen sich die räumlichen Gegebenheiten und die Ausstattung der Behandlungs- und Überwachungsräume an den Erfordernisse von Patienten mit relevanten Begleiterkrankungen (ASA III und höher) orientieren. Der Behandlungsraum sollte mit Monitoring (Pulsoximetrie, RR, EKG), Medikamenten, Sauerstoffanschluss, Absaugung und den Hilfsmitteln und Gerätschaften für die Durchführung einer Reanimation ausgestattet sein. Im Fall einer schwerwiegenden Komplikation muss eine geeignete Transportmöglichkeit in eine qualifizierte Behandlungseinrichtung (Intensivstation) sichergestellt sein (z.B. Aufzug mit Möglichkeit des Liegendtransportes)“. Derzeit liegt zu den gültigen Empfehlungen und Standards keine evidenzbasierte Datenlage vor.

Das BGH-Urteil Aktenzeichen: VI ZR265/02 hat das Sitzen von Patienten auf dem Flur, nach erfolgter Endoskopie unter Sedierung bemängelt. Der Aufwachbereich muss als solcher definiert sein und der Patient unter ständiger Aufsicht stehen.

\subsection{Apparative Ausstattung}

\subsubsection{Klinische Überwachung/Standard-Monitoring} Empfehlung

$\mathrm{Zu}$ den notwendigen Maßnahmen des Monitorings gehören die Pulsoxymetrie und die Blutdruckmessung (insbesondere bei Sedierung mit Propofol). Bei Patienten mit schwerer Herzerkrankung sollte darüber hinaus eine EKG-Registrierung erfolgen. Empfehlungsgrad: A, Evidenzstärke 5, starker Konsens.

\section{Anmerkung}

Die für die Überwachung zuständige Person kontrolliert klinisch die Atemtätigkeit des Patienten durch Beobachtung, durch Palpation der Thorax- und Bauchdeckenbewegungen und evtl. durch Palpation des Luftstroms der Ausatmung. Voraussetzung für das Endoskopie-Personal ist eine entsprechende Notfallausbildung und die Beherrschung der kardiopulmonalen Reanimation.

Entsprechend den Empfehlungen von verschiedenen nationalen und internationalen Fachgesellschaften [9-11, 19, 22, 37] wird eine pulsoximetrische Überwachung bei allen Untersuchungen gefordert. Bei sedierten Patienten und Risikopatienten werden ergänzend noch eine kontinuierliche Blutdruckkontrolle und eine EKG-Ableitung gefordert.

Neben der klinischen Überwachung ist die Pulsoxymetrie heute (insbesondere nach Einführung der Qualitätssicherungsver- einbarung zur Koloskopie gem. §135) Voraussetzung für eine Sedierung. Hierbei werden Sauerstoffsättigung und Herzfrequenz ständig gemessen. In der Schweiz wurden laut einer Umfrage 2003 über 95\% aller Endoskopien durch die Pulsoxymetrie überwacht [190]. In einer internationalen Studie in europäischen Zentren wurden 2006 die Koloskopien zu 77\% durch Pulsoxymetrie überwacht [191]. In einer aktuellen Umfrage in Deutschland wird die Pulsoxymetrie bei 97\% der Untersuchungen angewandt [5]. Bei der Sedierung mit Propofol ist auch eine Überwachung des Blutdrucks erforderlich. Optimal sind Überwachungsgeräte, bei denen Sauerstoffsättigung, Herzfrequenz und automatische Blutdruckmessung auf einem Monitor angezeigt werden, der direkt neben dem EndoskopieMonitor angebracht ist und somit vom Untersucher mitüberwacht werden kann. Auch eine Dokumentation der Messparameter ist wünschenswert.

\subsubsection{Erweitertes Monitoring \\ 3.5.2.1. Kapnografie \\ Empfehlung}

Die Kapnografie ist nicht obligat für die gastrointestinale Endoskopie; sie kann zur frühzeitigen Detektion einer Apnoe verwendet werden.

Empfehlungsgrad: 0, Evidenzstärke 2b, starker Konsens.

\section{Anmerkung}

Bei der Kapnografie wird über eine Nasenbrille die $\mathrm{CO}_{2}$-Konzentration in der Atemluft registriert. Dabei kann die Atmung grafisch dargestellt werden. Eine Apnoe kann dabei viel früher als mit der Pulsoximetrie diagnostiziert werden, was v.a. bei pädiatrischen Eingriffen von Bedeutung ist [192-194].

In einer prospektiven Studie von Vargo et al. [195] bei $49 \mathrm{Er}$ wachsenen zur Gastroskopie in therapeutischer Intention wurden durch die Kapnografie doppelt so viele Apnoe-Episoden diagnostiziert als durch Pulsoxymetrie und klinische Beobachtung. In einer weiteren prospektiven Studie von Anderson et al. [195] bei 163 Kindern wurde keine der bei 24\% der Endoskopien beobachteten Apnoe-Episoden durch alleinige Pulsoxymetrie und klinische Beobachtung diagnostiziert.

In einer retrospektiven Studie von Koniaris et al. [196] wurden 4846 Patienten ohne und 600 Patienten mittels Kapnografie im Rahmen der Endoskopie überwacht. In der Patientengruppe ohne Überwachung mittels Kapnografie kam es insbesondere bei älteren Patienten und lang dauernden Untersuchungen tendenziell, wenn gleich nicht signifikant häufiger zu einer Übersedierung als bei Verwendung der Kapnografie. Hier wurde kein Fall von Überdosierung beschrieben.

Darüber, ob durch Nichtanwendung der Kapnografie in der gastrointestinalen Endoskopie Schäden durch Hypoventilation entstehen, existieren keine Daten.

Anhand der derzeitig existenten Datenlage kann der routinemäßige Einsatz der Kapnografie nicht empfohlen werden.

\subsubsection{Monitoring mittels EEG}

Empfehlung

Der Nutzen des EEG-Monitorings hinsichtlich relevanter Zielgrößen in der gastrointestinalen Endoskopie ist nicht nachgewiesen. Empfehlungsgrad: 0, Evidenzstärke 1b, starker Konsens.

\section{Anmerkung}

Das bispektrale Monitoring wird in der Intensivmedizin und bei chirurgischen Patienten verwandt, um die Sedationstiefe zu eva- 
luieren. In einer Pilotstudie wurde das bispektrale Monitoring als Teil eines geschlossenen Überwachungskreislaufs für die Dauerinfusion mit Propofol bei 16 für die Koloskopie sedierten Patienten beschrieben [84]. In einer weiteren randomisiert, kontrollierten Studie von Wehrmann et al. [115] konnte bei der Verwendung eines andersartigen EEG-Monitorings im Rahmen der ERCP eine signifikante Verminderung der benötigten Propofol-Dosis gezeigt werden. Für die gastrointestinale Endoskopie kann das bispektrale Monitoring, insbesondere bei kurz dauernden Untersuchungen wie z.B. der Koloskopie, nicht als Überwachungsmethode empfohlen werden, da hier anhand der vorliegenden Studiendaten $[197,198]$ keine Einsparung der Propofol-Dosis und entsprechend schnellere Erholungszeit erzielt wurde.

\section{Aufklärung, Einwilligung/Voraussetzung für Durchführung der Sedierung/Sicherung vitaler Funktionen/klinische Überwachung/Zwischenfall- management

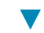

4.1. Patientenaufklärung und -einwilligung Empfehlung

Die Patienten sollen im Rahmen der Aufklärung über die Endoskopie auch über sedierungsassoziierte Wirkungen, insbesondere retrograde Amnesie und die Möglichkeit eingeschränkter psychomotorischer Leistungen nach der Sedierung aufgeklärt sein. Empfehlungsgrad: A, Evidenzstärke 5, starker Konsens.

\subsubsection{Allgemeine und rechtliche Aspekte}

Die Rechtsprechung zum ärztlichen Eingriff, der ärztlichen Aufklärung und der Patientenaufklärung ist äußert komplex.

Nach der ständigen Rechtssprechung des Bundesgerichtshofs stellt jeder ärztliche Eingriff den Tatbestand der Körperverletzung im Sinn des $\S \S 223$ ff. StGB; 823 I BGB dar. Ein ärztlicher Eingriff umfasst nicht nur die Durchführung diagnostischer Verfahren, sondern auch therapeutische Maßnahme wie eine Operation oder die Verabreichung von Medikamenten [199].

Die für diese Maßnahmen notwendige Einverständniserklärung ist nur wirksam, wenn der Patient hinreichend aufgeklärt wurde und von seinem Selbstbestimmungsrecht Gebrauch machen kann (,informend consent“).

Sollte der Patient einwilligungsunfähig sein (z. B. Kinder, schwer geistig behinderte Personen), muss der Arzt dessen Vertreter (Erziehungsberechtigte, Bevollmächtigte für Gesundheitsangelegenheiten oder Betreuer) aufklären [200, 201].

Der Patient ist nur dann einwilligungsfähig, wenn er auch die Tragweite des Eingriffs verstehen kann. Abseits des rechtlichen Hintergrunds erhöht eine gute Aufklärung auch die Patientenzufriedenheit [37].

Eine Aufklärung sollte auch erfolgen, wenn der Patient bereits früher endoskopische Eingriffe derselben Art erfahren hat oder angibt, gut informiert zu sein. Denn viele Patienten können ihr eigenes Informations- und Wissensniveau schlecht einschätzen. Ferner geraten frühere Aufklärungen in Vergessenheit oder Details der Aufklärung sind unverstanden geblieben.

\subsubsection{Aufklärende Person Empfehlung}

Die Aufklärung soll durch einen fach- und sachkundigen Arzt in für den Patienten verständlicher Form erfolgen.

Empfehlungsgrad: A, Evidenzstärke 5, starker Konsens.

\section{Anmerkungen}

Der Arzt muss über die notwendige Fach- und Sachkenntnis zur Sedierung verfügen. Die Aufklärung muss in einer für den Patienten - als medizinischen Laien - behutsamen und verständlichen Weise erfolgen. Wichtig ist, dass der Patient die Bedeutung und Tragweite des Eingriffs einschätzen kann. Davon hat sich der Arzt im Gespräch zu überzeugen. Eine Delegation, z.B. an ärztliches Hilfspersonal, ist nicht statthaft.

\subsubsection{Durchführung der Aufklärung \\ Empfehlung}

Grundlage der Aufklärung soll das Gespräch zwischen Arzt und Patient sein. Inhalt und Umfang der Aufklärung sollen dokumentiert werden. Die Aufklärung soll rechtzeitig erfolgen.

Empfehlungsgrad: A, Evidenzstärke 5, starker Konsens.

\section{Anmerkungen}

Das Gespräch muss patientenzentriert geführt werden, d.h. abhängig von der Auffassungsgabe und dem Umfang des Informationsbedürfnisses des Patienten. Standardisierte Aufklärungsbogen können zusätzlich verwandt werden, sie dienen aber nur der Unterstützung in der Informationsweitergabe und der Dokumentation und sind kein Ersatz für das persönliche Aufklärungsgespräch.

Zivilrechtlich hat der Arzt die Beweispflicht für die ordnungsgemäße Durchführung der Aufklärung, eine schriftliche Dokumentation ist deshalb essenziell [199].

Der Umfang der Aufklärung ist umgekehrt proportional zur Dringlichkeit des Eingriffs.

Ein Aufklärungsverzicht sollte dem Patienten darum nicht angeboten und erst recht nicht nahe gelegt werden. Wenn der Patient jedoch aus eigenem Antrieb eine Aufklärung ablehnt, ist der Aufklärungsverzicht zu dokumentieren und vom Patienten zu unterschreiben [201].

Die Bedeutung des Selbstbestimmungsrechts des Patienten verlangt zu dem Rechtzeitigkeit [202] der Einwilligungserklärung und damit auch eine Aufklärung, die eine Entscheidungsfreiheit ohne Zeitdruck gewährleistet.

Der richtige Zeitpunkt der Aufklärung hängt von den Umständen im Einzelfall ab. Ansprechbare Patienten sollten auch in Notfällen vor ihrer Einwilligung soweit aufgeklärt werden, wie es die Zeit zulässt [201].

Insgesamt sollte die Aufklärung so früh wie möglich erfolgen, es ist anzustreben, dass sie begonnen wird, wenn der Termin für den endoskopischen Eingriff vereinbart wird [201].

\subsubsection{Inhalte des Aufklärungsgesprächs \\ Empfehlung}

Das Gespräch soll Informationen über die Vorbereitung der Sedierung, verschiedene Sedierungsmethoden und deren mögliche Komplikationen umfassen. Inhalt des Gesprächs soll es auch sein, den Patienten auf die Möglichkeit hinzuweisen, auf die Sedierung zu verzichten.

Empfehlungsgrad: A, Evidenzstärke 5, starker Konsens.

\section{Anmerkungen}

In fast allen Untersuchungen sind unerwünschte Wirkungen der Sedierung für mindestens $50 \%$ der Komplikationen verantwortlich [203]. Somit sind die Sedierungskomplikationen (Aspiration, arterielle Hypotension, Bradykardie, Apnoe usw.) typische Komplikationen und müssen mit dem Patienten ausführlich besprochen werden. Über typische Risiken der Sedierung ist unabhängig 
von der Komplikationsrate aufzuklären. Auch gravierende atypische Risiken müssen aufgeklärt werden. Gleichzeitig muss die Aufklärung schonend erfolgen, der Patient darf nicht durch eine übertriebene Risikoaufklärung verängstigt werden.

Der Arzt sollte hinsichtlich des „Ob und Wie“ der Sedierung zum Berater des Patienten werden. Bezüglich der Dosis der Sedierung erscheint es sinnvoll, neben Geschlecht und Alter auch den Beunruhigungs-/Angstlevel der Patienten zu erheben, da sich diese 3 Faktoren als geeignet für die Vorhersage der Kooperation des Patienten während der Untersuchung und seiner Zufriedenheit danach erwiesen haben [105, 204, 205]. Jüngeren und ängstlicheren Patienten sowie Frauen sollte eher zu einer Sedierung geraten werden [20, 27, 30, 48, 105].

Da aber gerade bei länger dauernden Untersuchungen und diffizilen interventionellen Eingriffen (z.B. ERCP, schwierige Polypektomie) die Vermeidung von ungewollten Spontanbewegungen der Patienten durch eine Sedierung in der Regel erforderlich $[11,23,91,206]$ erscheint, sollte dies auch den Patienten gegenüber begründet werden.

Kommt der Patient zu Schaden, indem er die Zustimmung aufgrund einer zu „harten“ Aufklärung zu einer notwendigen Untersuchung verweigert, trägt der Arzt die Verantwortung [207]. Wenn entweder der Patient in eine hohe ASA-Risikoklasse fällt [37] oder wenn die Einrichtung und das Personal nicht ausreichen, um die Sedierung fachgerecht durchzuführen, kann eine Sedierung auch abgelehnt werden. In diesen Fällen muss der Arzt dem Patienten erklären, warum keine Sedierung erfolgen kann.

Ob über die Möglichkeit des Todes durch den Eingriff aufgeklärt werden muss, ist umstritten, in 2 Urteilen der Oberlandesgerichte Stuttgart $[207,208]$ und einem Urteil des Oberlandesgerichts Zweibrücken [209] wurde gefordert, dass der Patient vor der Koloskopie schonend darüber informiert werden müsste, dass er an den Folgen einer Perforation versterben könnte. Im anderen Fall wurde eingefordert, dass die Patientin darüber informiert wurde, dass sie durch eine ERCP versterben kann.

\subsubsection{Sicherungsaufklärung (Verhalten nach der Sedierung) Empfehlung}

Der Patient soll über das korrekte Verhalten nach der Sedierung und der Entlassung aus der ambulanten Therapie aufgeklärt werden. Er soll ein Informationsblatt ausgehändigt bekommen. Empfehlungsgrad: A, Evidenzstärke 5,starker Konsens.

\section{Anmerkungen}

Verhalten nach der Untersuchung (Sicherungsaufklärung): Ambulant untersuchte Patienten sollten darauf hingewiesen werden, dass sie einer Begleitperson bedürfen, die sie zumindest sicher nach Hause bringt und sie möglichst auch für einige Stunden beobachtet. Alle Patienten, die eine Sedierung erhalten haben, sind darüber hinaus darauf hinzuweisen, dass sie am Untersuchungstag nicht aktiv am Straßenverkehr teilnehmen dürfen, insbesondere nicht ein Fahrzeug führen, keine schwierigen Maschinen bedienen, keinen Alkohol trinken und keine wichtigen oder rechtlich bindenden Entscheidungen treffen dürfen. Grundsätzlich ist allen Patienten eine telefonische Kontaktmöglichkeit zum Arzt oder Klinikum zu benennen für den Fall, dass nach der Endoskopie Befindlichkeitsstörungen oder Blutungen auftreten [11, 23, 201, 210]. Die Aufklärungen über die Verhaltensmaßnahme für die Zeit nach der Untersuchung sollten dem Patienten schriftlich an die Hand gegeben werden [11, 201, 210]. Bei kritischen Prozeduren oder Patienten war hier ein Telefonanruf am nächsten Tag wirksamer als eine schriftliche Erinnerung [211].

\subsection{Voraussetzung für die Durchführung der Sedierung Empfehlung \\ Voraussetzung für eine Sedierung ist ein permanenter intravenöser Zugang. \\ Empfehlungsgrad: A, Evidenzstärke 2b, starker Konsens.}

\section{Anmerkung}

Die Verabreichung von Sedativa erfordert prinzipiell einen peripher-venösen Zugang.

In einer vergleichenden Studie von Smith et al. [212] zur Funktionstüchtigkeit von Butterfly und Venenverweilkanüle $1 \mathrm{~h}$ nach erfolgter Endoskopie, also einem Zeitraum, in dem die Halbwertszeit der meisten Benzodiazepine und Opiate nicht einmal erreicht ist, waren nur noch $44 \%$ der Butterflys funktionstüchtig, während 98\% der Venenverweilkanülen noch funktionstüchtig waren.

\subsection{Sicherung vitaler Funktionen \\ Empfehlung \\ Sedierte Patienten sollen prophylaktisch Sauerstoff über eine Na- sensonde erhalten. \\ Empfehlungsgrad: A, Evidenzstärke 2b, starker Konsens.}

\section{Anmerkung}

Zwischenfälle, die auf eine Sedierung zurückzuführen sind, sind in erster Linie kardiopulmonale Ereignisse [203]. Diese machen etwa die Hälfte aller Komplikationen in der Endoskopie aus und können je nach Risiko des Patienten bei Gastroskopien auch ohne Sedierung auftreten [213]. Die Häufigkeit kardiopulmonaler Ereignisse wird in der älteren Literatur und unter Verwendung von Benzodiazepinen um 5\% beziffert [124]. In neueren Studien mit Propofol zwischen 0,0 und 0,65\% und in einer großen Beobachtungsstudie über die Vorsorge-Koloskopie aus Deutschland mit $0,1 \%$, wobei $80 \%$ der Untersuchungen mit Sedierung durchgeführt wurden, eine Spezifikation der Medikamente aber nicht angegeben war [214]. Besonders ältere Patienten sind für eine Hypoxie unter Sedierung gefährdet $[13,103]$.

Eine prophylaktische Sauerstoffgabe über eine Nasensonde kann die Häufigkeit hypoxämischer Ereignisse signifikant vermindern [215 - 217]. Allerdings gibt es auch Hinweise, dass die prophylaktische Sauerstoffgabe die frühzeitige Detektion von hypoxämischen Ereignissen mittels Pulsoxymetrie verzögern kann [20].

\subsection{Zwischenfallmanagement}

4.4.1. Hypoxie

Eine Hypoxie liegt vor bei einem Abfall der Sauerstoffsättigung unter 90\%, gemessen mit dem Pulsoximeter. Bei den meisten Pulsoxymetern wird die Höhe der Sauerstoffsättigung durch die Tonhöhe des Impulses angezeigt. Sinkt also die Tonfrequenz oder die digital sichtbare Sauerstoffsättigung kontinuierlich ab, müssen Gegenmaßnahmen getroffen werden. Diese bestehen zunächst darin, dass man den Patienten laut auffordert und durch taktile Reize anregt, tiefer zu atmen. In Rückenlage kann durch Esmarch-Handgriff das Kinn nach oben gezogen werden, sodass der Patient wieder frei durch den Mund atmen kann, evtl. hilft hier das Einlegen eines Güdel- oder Wendel-Tubus. Außerdem sollte der Sauerstofffluss erhöht werden (z.B. von 2 auf $4-51 / \mathrm{min}$ ). 
Sollte der Patient unter diesen Maßnahmen keine Spontanatmung entwickeln, muss die Atemhilfe weiter fortgesetzt werden. Der Atemweg ist dann instrumentell zu sichern (z.B. Intubation).

Im Falle einer Sedierung mit Benzodiazepinen ist zusätzlich sofort der Antagonist Flumazenil intravenös zu verabreichen, wodurch eine Beatmung häufig vermieden werden kann. Ansonsten soll bei einer Hypoxie unter Benzodiazepinen gleich verfahren werden wie unter Propofol.

\subsubsection{Herzrhythmusstörungen}

\subsubsection{Allgemeines}

Allein die endoskopische Intubation des Kolons erzeugt beim unsedierten Patienten ein Übergewicht des sympathischen autoregulativen Nervensystems [218] und erhöht damit die Wahrscheinlichkeit für kardiovaskuläre Ereignisse. Der Einfluss auf die Herzfrequenz-Variabilität wird aber durch eine Sedierung noch verstärkt [219]. Kardiopulmonale Ereignisse können aber auch bei unsedierten Patienten während der Gastroskopie beobachtet werden [140].

\subsubsection{Tachykarde Herzrhythmusstörungen}

Über interventionspflichtige Tachykardien wird in der Literatur nicht berichtet, lediglich über gehäufte supraventrikuläre und ventrikuläre Extrasystolen [220]. Im Notfall sollten Antiarrhythmika der Klasse Ia-IV und ein Defibrillator nach den Leitlinien der kardiologischen Fachgesellschaften [221] bereitgehalten werden.

\subsubsection{Bradykarde Herzrhythmusstörungen}

Insbesondere bei der Koloskopie mit oder ohne Sedierung treten mitunter Bradykardien auf. Die Häufigkeit wird mit 0,5\% angegeben [189], wobei aber nur bei einem Drittel der Patienten eine medikamentöse Intervention erforderlich war. Die Intervention besteht aus der Gabe von 0,5 mg Atropin i.v., evtl. wiederholt bis $3 \mathrm{mg}$ und/oder Adrenalin 0,02-0,1 mg i.v., in bedrohlichen Fällen Reanimation mit Herzmassage [221].

\subsubsection{Arterielle Hypotonien}

Die Häufigkeit einer arteriellen Hypotension bei Koloskopie schwankt je nach Definition zwischen 0,3\% [189] und $3-19 \%$ [218]. Im Falle einer arteriellen Hypotension besteht die Intervention in der Infusion von physiologischer Kochsalzlösung. Die prophylaktische Infusion bei allen Koloskopien wird nicht empfohlen [222], ist aber bei älteren exsikkierten Patienten durchaus sinnvoll. Auch bei länger dauernden Eingriffen unter Propofol-Sedierung kann, wegen der ausgeprägten blutdrucksenkenden Eigenschaften von Propofol, die prophylaktische intravenöse Gabe von Flüssigkeit als sinnvoll erachtet werden.

\subsubsection{Myokardiale Ischämien}

Myokardiale Ischämien können während der Endoskopie bei sedierten und unsedierten Patienten auftreten. In einer prospektiven Studie wurde eine ST-Segmentdepression in 7\% der koloskopierten Patienten beschrieben, wobei $3 / 4$ der Ereignisse vor der eigentlichen Endoskopie auftraten [223]. ST-Senkungen können durch Sauerstoff-Supplementation während der Endoskopie signifikant verringert werden [217]. In der Literatur wird ein Fall eines Herzinfarkts während der Koloskopie beschrieben [155].

\subsubsection{Seltene Ereignisse bei Sedierung}

Selten werden bei Endoskopien von sedierten Patienten Allergien oder lokale Schmerzreaktionen (meist bei Injektion in kleine Venen) beobachtet. Bei 80000 Koloskopien wurde eine allergische Reaktion gegen Midazolam beobachtet [205].

\section{Qualitätsziele: Interne Qualitätssicherung/Entlas- sungskriterien/ „Street-Fitness“|Arbeitsfähigkeit| Dokumentation/„Benchmarking“ $\nabla$}

Um eine hohe Ergebnissqualität in einem Prozess erreichen zu können, bedarf es zunächst der Festlegung und Einhaltung von Qualitätszielen.

Die Moderation des Entwicklungsprozesses von Qualitätsmessverfahren folgt einem fest strukturierten Ablauf und beginnt mit der Definition der Qualitätsziele. Qualitätsziele können sich auf den Versorgungsprozess oder auf das Behandlungsergebnis beziehen. In der internationalen Literatur wird anstelle des Qualitätsziels oft ein „Indicator statement“ festgelegt.

Am Anfang steht die Ergänzung des Halbsatzes „Gute Qualität ist, wenn"

- Patienten sich durch optimale Aufklärung selbstständig für oder gegen eine Sedierungsform entscheiden können,

- Patienten eine adäquate, individuell an die Bedürfnisse und den Gesundheitszustand angepasste Sedierung im Rahmen der gastrointestinalen Endoskopie erhalten,

- eine hohe Patientenakzeptanz für endoskopische Untersuchungen insbesondere im Rahmen der Vorsorge erzielt werden kann,

- Endoskopiker optimale Untersuchungsbedingungen durch die Sedierung haben,

- Patienten durch ein adäquates Monitoring während der Sedierung keine Komplikationen erleiden bzw. eine hohe Patientensicherheit durch Minimierung der sedierungsbezogenen Komplikationsrate erzielt werden kann,

- Patienten im Falle von unvorhersehbaren Komplikationen nach den Regeln der Wissenschaft schnell und sorgfältig behandelt werden,

- Patienten bis zur Entlassung ausreichend betreut werden,

- Patienten erst entlassen werden, wenn durch die u.U. noch nicht vollständig abgeklungene Wirkung der Medikamente keine schwerwiegende Beeinträchtigung mehr nachweisbar ist.

Die oben benannten Punkte sollen zur Optimierung und Einhaltung der definierten Qualitätsziele beitragen.

Da dies die erste Version einer S3-Leitlinie zur Sedierung in der gastrointestinalen Endoskopie ist und für dieses Thema nur eine sehr begrenzte Datenlage zur Verfügung steht, wurden über die Qualitätsziele hinaus noch keine expliziten Indikatoren mit Referenzbereichen definiert.

Die Leitliniengruppe befand, dass hierfür zunächst umfassende Daten aus der Versorgungspraxis erhoben werden sollten, um zu prüfen, inwieweit die hier vorgeschlagenen Qualitätsziele bereits abgebildet werden. Anhand dieser Ergebnisse ist eine weitere Konkretisierung der Qualitätsindiaktoren entsprechend den BQS-Verfahren (siehe www.bqs-online.de, Bundesgeschäftsstelle für Qualitätssicherung) im Rahmen der Aktualisierung angestrebt.

\subsection{Interne Qualitätssicherung Empfehlung \\ Es soll eine schriftliche und klar verständliche Ablaufplanung für die Durchführung von Sedierungen, die Überwachung der Patien-}


ten nach der Sedierung, die Entlassungskriterien in den ambulanten bzw. allgemein-stationären Bereich sowie hinsichtlich eines möglichen Komplikationsmanagements vorliegen. Die jeweiligen Zuständigkeiten sollen hierbei klar definiert sein.

Empfehlungsgrad: A, Evidenzstärke 5, starker Konsens.

\subsection{Entlassungskriterien}

5.2.1. Patienteninstruktionen

Empfehlung

Die Entlassung der Patienten sollte in Begleitung erfolgen. Weiterhin soll der Patient schriftliche Instruktionen einschließlich einer 24-h-erreichbaren Notfalltelefonnummer für den Fall von Komplikationen erhalten.

Empfehlungsgrad: A, Evidenzstärke 5, starker Konsens.

\subsubsection{Minimalkriterien für die Entlassung}

Empfehlung

Die Minimalkriterien für die Entlassung (gemäß des „Report of the Working Party on Guidelines for Sedation by Non-Anaesthetists“ [225]) sollen erfüllt und dokumentiert sein.

Empfehlungsgrad: A, Evidenzstärke 5, starker Konsens ( $\bullet$ Tab. 13).

\subsubsection{Verwendung von Score-Systemen für die Entlassung Empfehlung}

Score-Systeme (z.B. Aldrete-Score) sollten nicht alleine zur Beurteilung der Entlassungsfähigkeit verwendet werden, da sie die psychomotorische Funktion der Patienten nicht valide beurteilen können. Empfehlungsgrad: B, Evidenzstärke 2b, starker Konsens.

\section{Anmerkung}

Die Verwendung eines modifizierten Aldrete-Scores [226] ermöglicht nur Aussagen zu den Vitalfunktionen des Patienten, aber nicht zur psychomotorischen Leistungsfähigkeit. Diese ist bei Erreichen eines maximalen Score-Wertes noch erheblich eingeschränkt [227].

Auch wenn Patienten nach einer Sedierung stabile Vitalzeichen zeigen und ausreichend wach erscheinen, ist bekannt, dass bei Verwendung mittellang wirkender Substanzen (z. B. Dormicum, Pethidin) eine verlängerte Phase von Amnesie und eine Beeinträchtigung des Urteilsvermögens sowie der Reflexe vorhanden sind.

Bei Patienten, die im Rahmen einer Koloskopie mit dem häufig verwendeten Sedierungsregime Midazolam plus Opiat sediert werden, sind Reaktionszeit, Feinmotorik und die Wahrnehmung zumindest für 30 min nach der Untersuchung eingeschränkt. Die Studiendaten zeigen, dass die verbleibenden Nachwirkungen von Midazolam unterschiedliche Aspekte der psychomotorischen Funktion für mindestens eine Stunde nach der Applikation beeinträchtigen [227, 228]. Midazolam scheint hier der Hauptgrund für die anhaltende psychomotorische Funktionseinschränkung nach Sedierung zu sein [229]. Ein ähnliches Ergebnis erbrachte auch die Studie von Thapar et al. [229], in der die Wirkung von Midazolam mit Fentanyl und Propofol verglichen wurde.

\section{3. „Street-Fitness“}

5.3.1. Verkehrstauglichkeit

Empfehlung

Die aktive und passive Teilnahme am Straßenverkehr soll in Abhängigkeit von der Halbwertszeit der verwendeten Substanzen und vom Risikoprofil des Patienten zum Entlassungszeitpunkt individuell entschieden werden.

Empfehlungsgrad: A, Evidenzstärke 1b, starker Konsens.
Tab. 13 Minimalkriterien für die Entlassung nach sedierter Endoskopie (nach [225]).

Gehen ohne Hilfe
komplette (oder weitgehende) Schmerzfreiheit
orale Flüssigkeitsaufnahme ohne Schwierigkeiten
fehlende oder minimale Übelkeit
ausreichende Nachsorge zu Hause gegeben
ggf. nochmaliger Hinweis auf typische Anzeichen von Komplikationen
durch den Arzt, Notfalltelefonnummer mitgeben
Entlassung in Begleitung

\section{Anmerkung}

Die Normalisierung der psychomotorischen Funktion am Untersuchungstag hängt entscheidend von der Halbwertszeit der verwendeten Substanz ab, wobei kurz wirksame Substanzen einen Vorteil bieten.

In einer Studie von Riphaus et al. [8] an 98 Patienten, die im Rahmen der Gastroskopie und Koloskopie entweder mittels Propofol oder Midazolam/Pethidin sediert wurden, zeigte sich $2 \mathrm{~h}$ nach der Sedierung mit Propofol im Vergleich zu Midazolam/Pethidin keine Einschränkung der psychomotorischen Fähigkeiten (unter Verwendung eines Fahrsimulators).

Nach Verwendung von kurz wirksamen Hypnotika (z. B.Propofol) scheint unter Berücksichtigung der Halbwertszeit die Verkehrstauglichkeit wiederhergestellt zu sein. Gleichwohl wird aufgrund der in der vorliegenden Studie geringen Fallzahlen derzeit allenfalls die Benutzung von Bus und Bahn (auch ohne Begleitperson) für möglich gehalten. Für das Führen eines Kraftfahrzeugs/Fahrrads bedarf es jedoch weiterer großer Feldstudien.

Die derzeit geltenden Empfehlungen der einzelnen Fachgesellschaften [9-11], für $24 \mathrm{~h}$ weder aktiv noch passiv am Straßenverkehr teilzunehmen, erscheint bei fehlender Evidenz für die Festlegung dieses Zeitraums, insbesondere bei der Verwendung von ultrakurz wirksamen Substanzen, wie Propofol und Remifentanil, als zu weit gefasst.

Bereits in einer ganzen Reihe von Studien von Kortilla et al. [230-232] aus den 70er-Jahren, in denen die psychomotorischen Fähigkeiten nach Sedierung mit verschiedenen Substanzen untersucht wurde, konnte gezeigt werden, dass selbst bei der Verwendung von Benzodiazepinen in einer höheren Dosierung (Diazepam $0,45 \mathrm{mg} / \mathrm{kg} / \mathrm{KG}$ ) die psychomotorischen Funktionen bereits nach $10 \mathrm{~h}$ nicht mehr eingeschränkt waren [231]. Lediglich bei der heute im Rahmen der Endoskopie nicht mehr üblichen Verwendung von $75 \mathrm{mg}$ Dolantin i. m. zeigten sich die psychomotorischen Fähigkeiten für bis zu $12 \mathrm{~h}$ eingeschränkt, sodass hier die Empfehlung eines „Fahrverbots“ für $24 \mathrm{~h}$ gerechtfertigt erscheint [230].

\subsection{Dokumentation \\ Empfehlung}

Die Akte oder das Dokumentationsblatt soll eine zeitabhängige Dokumentation der Vitalparameter (Sauerstoffsättigung, Herzfrequenz und Blutdruck), der verwendeten Medikamente mit Namen und Dosierung sowie der Gabe intravenöser Flüssigkeit enthalten und Angaben darüber machen, ob und in welcher Flussrate der Patient Sauerstoff erhalten hat. Idealerweise sollen periodisch auch der Sedierungsgrad und Schmerzangaben des Patienten dokumentiert werden.

Empfehlungsgrad: A, Evidenzstärke 5, starker Konsens. 


\subsubsection{Allgemeines}

Die Dokumentation ist ein essenzieller Bestandteil der Patientenfürsorge und soll während aller Phasen der Untersuchung durchgeführt werden.

Hierzu zählen

- die präinterventionelle Einschätzung des Patienten,

- die Aufklärung des Patienten,

- das Monitoring während der Untersuchung,

- die Patientenerholung,

- die Patientenentlassung.

Idealerweise sollte hier ein standardisierter Dokumentationsbogen verwandt werden, da hierdurch die Einhaltung der Dokumentation verbessert werden kann [233].

\subsubsection{Arbeitsunfähigkeit}

\section{Empfehlung}

Die Dauer einer Arbeitsunfähigkeit soll individuell, arbeitsplatzkonkret und unter Berücksichtigung der Dauer und Tiefe der Sedierung sowie der verwendeten Substanz beurteilt werden. Empfehlungsgrad: 0, Evidenzstärke 5, Konsens.

\section{Anmerkung}

Eine generelle Empfehlung über die Dauer einer Arbeitsunfähigkeit kann nach der Verwendung von Sedativa und Analgetika im Rahmen gastrointestinaler Endoskopien nicht abgegeben werden. Die üblichen Empfehlungen einer generellen Arbeitsunfähigkeit von 24 Stunden nach einer Sedierung können bei Verwendung kurzwirksamer Pharmaka als zu weit gefasst angesehen werden.

\subsection{Benchmarking \\ Empfehlung}

Alle Komplikationen (Abfall der Sauerstoffsättigung, Hypotonien, Maskenbeatmungen, Inkubationen und Todesfälle), die bei der Endoskopie mit und ohne Sedierung auftreten, sollten dokumentiert werden.

Empfehlungsgrad: 0, Evidenzstärke 5, Konsens.

\section{Anmerkungen}

„Benchmark“ bedeutet übertragen die Orientierungsgröße (Kennzahl) bzw. die Gesamtheit der Vergleichsgrößen für eine relative Bewertung eines Produkts, einer Dienstleistung oder einer Organisationseinheit im wettbewerblichen Vergleich.

Die wesentliche Orientierungsgröße bei der Sedierung im Rahmen der gastrointestinalen Endoskopie sollte eine möglichst geringe Komplikationsrate sein.

Eine bundesweite Erhebung sämtlicher endoskopischer Untersuchungen und assoziierter Komplikationen mit und ohne Sedierung ist erstrebenswert.

Die o.a. Komplikationen sollten zentral registriert und entsprechend ausgewertet werden, um nachfolgend Prozessabläufe optimieren zu können.

\section{Literatur}

1 Daneshmend TK, Bell GD, Logan RF. Sedation for upper gastrointestinal endoscopy: results of a nationwide survey. Gut 1991; 32: 12-15

2 Keefe EB, O'Connor KW. ASGE survey of endoscopic sedation and monitoring practices. Gastrointest Endosc 1990; 36: 13-18

3 Froehlich F, Gonvers JJ, Fried M. Conscious sedation, clinically relevant complications and monitoring of endoscopy: results of a nationwide survey in Switzerland. Endoscopy 1994; 26: 231-234
4 Frühmorgen P, Kriel L. Guidelines of the German Society of Digestive and Metabolic Diseases. Guidelines for endoscopic colorectal polypectomy with the sling. German Society of Digestive and Metabolic Diseases. Gastroenterol 1998; 36: 117-119

5 Riphaus A, Rabofski M, Wehrmann T. Sedierung in der gastrointestinalen Endoskopie in Deutschland. Z-Gastroenterol 2007; 45: 782

6 Carlsson U, Grattidge P. Sedation for upper gastrointestinal endoscopy: a comparative study of propofol and midazolam. Endoscopy 1995; 27: 240-243

7 Patterson KW, Casey PB, Murray JP et al. Propofol sedation for outpatient upper gastrointestinal endoscopy: comparison with midazolam. Br J Anaesth 1991; 67: 108-111

8 Riphaus A, Gstettenbauer T, Frenz MB et al. Quality of psychomotor recovery after propofol sedation for routine endoscopy: a randomized and controlled study. Endoscopy 2006; 38: 677-683

9 Clinical Practice Guidelines: Safety and Sedation During Endoscopic Procedures. Gut 2003. http://www.bsg.org.uk/pdf_word_docs/sedation.doc

10 Stufe 1 Leitlinie Sedierung und Analgesie (Analgosedierung) von Patienten durch Nicht-Anästhesisten. 2008. http://www.dgai.de/ 06pdf/13_573-Leitlinie.pdf, http://intranet/awmf11/001 - 011.htm

11 Hofmann C, Jung $M$. Sedierung und Überwachung bei endoskopischen Eingriffen. 2003. http://www.dgvs.de/media/1.2.Sedierungueberwachung.pdf

12 Kulling $D$, Rothenbuhler $R$, Inauen $W$. Safety of nonanesthetist sedation with propofol for outpatient colonoscopy and esophagogastroduodenoscopy. Endoscopy 2003; 35: 679-682

13 Heuss LT, Drewe J, Schnieper P et al. Patient-controlled versus nurseadministered sedation with propofol during colonoscopy. A prospective randomized trial. Am J Gastroenterol 2004; 99: 511-518

14 Heuss LT, Schnieper P, Drewe J et al. Risk stratification and safe administration of propofol by registered nurses supervised by the gastroenterologist: a prospective observational study of more than 2000 cases. Gastrointest Endosc 2003; 57: 664-671

15 Rex DK, Heuss LT, Walker JA et al. Trained registered nurses/endoscopy teams can administer propofol safely for endoscopy. Gastroenterology 2005; 129: 1384-1391

16 Rex DK, Overley C, Kinser K et al. Safety of propofol administered by registered nurses with gastroenterologist supervision in 2000 endoscopic cases. Am J Gastroenterol 2002; 97: 1159-1163

17 Walker JA, McIntyre RD, Schleinitz PF et al. Nurse-administered propofol sedation without anesthesia specialists in 9152 endoscopic cases in an ambulatory surgery center. Am J Gastroenterol 2003; 98: $1744-1750$

18 Tohda G, Higashi S, Wakahara S et al. Propofol sedation during endoscopic procedures: safe and effective administration by registered nurses supervised by endoscopists. Endoscopy 2006; 38: 360-367

19 American Society of Anesthesiologists Task Force. Practice guidelines for sedation and analgesis by non-anesthesiologists: an updated report by the American Society of Anestesiologists Task Force on Sedation and Analgesia by Non- Anesthesiologists. Anesthesiology 2002; 96: 1004-1017

20 American Society for Gastrointestinal Endoscopy. Guidelines for conscious sedation and monitoring during gastrointestinal endoscopy. Gastrointestinal Endoscopy 2003; 58: 317-322

21 American Society of Anesthesiologist. Practice guidelines for postanesthetic care: a report by the American Society of Anesthesiologists Task Force on Postanesthetic Care. Anesthesiology 2002; 96: 742-752

22 Joint statement of a Working from the American College of Gastroenterology (ACG), the American Gastroenterological (AGA), and the American Society for Gastrointestina Endoscopy (ASGE). Recommendations on the administration of sedation for the performance of endoscopic procedures. 2006. www.gi.org/physicians/nataffairs/trisociety.asp

23 Schreiber F. Austrian Society of Gastroenterology and Hepatology (OGGH) - guidelines on sedation and monitoring during gastrointestinal endoscopy. Endoscopy 2007; 39: 259-262

24 American Society for Gastrointestinal Endoscopy. Guidelines for training in patient monitoring and sedation and analgesia. Gastrointest Endosc 1998; 48 (6): 669-671

25 Waring JP, Baron TH, Hirota WK et al. Guidelines for conscious sedation and monitoring during gastrointestinal endoscopy. Gastrointest Endosc 2003; 58: 317-322 
26 Probert CS, Jayanthi V, Quinn J et al. Information requirements and sedation preferences of patients undergoing endoscopy of the upper gastrointestinal tract. Endoscopy 1991; 23: 218-219

27 Rex DK, Imperiale TF, Portish V. Patients willing to try colonoscopy without sedation: associated clinical factors and results of a randomized controlled trial. Gastrointest Endosc 1999; 49: 554-559

28 Eckardt VF, Kanzler G, Schmitt T et al. Complications and adverse effects of colonoscopy with selective sedation. Gastrointest Endosc 1999; 49: 560-565

29 Yoruk G, Aksoz K, Unsal B et al. Colonoscopy without sedation. Turk J Gastroenterol 2003; 14: 59-63

30 Early DS, Saifuddin T, Johnson JC et al. Patient attitudes toward undergoing colonoscopy without sedation. Am J Gastroenterol 1999; 94: 1862-1865

31 Cohen LB, Wecsler JS, Gaetano JN et al. Endoscopic sedation in the United States: results from a nationwide survey. Am J Gastroenterol 2006; 101: 967-974

32 Thompson DG, Lennard-Jones JE, Evans SJ et al. Patients appreciate premedication for endoscopy. Lancet 1980; 2: 469-470

33 Dillon M, Brown S, Casey W et al. Colonoscopy under general anesthesia in children. Pediatrics 1998; 102: 381-383

34 Ely EW et al. Monitoring sedation status over time in ICU patients: reliability and validity of the Richmond Agitation-Sedation Score (RASS). JAMA 2003; 289: 2983-2991

35 Cohen LB, Delegge $M H$, Aisenberg J et al. AGA Institute review of endoscopic sedation. Gastroenterology 2007; 133: 675-701

36 Dripps RD, Lamont A, Eckenhoff JE. The role of anesthesia in surgical mortality. JAMA 1961; 178: 261-266

37 American Society of Anesthesiologists. Practice guidelines for sedation and analgesia by non-anesthesiologists. A report by the American Society of Anesthesiologists Task Force on Sedation and Analgesia by Non-Anesthesiologists. Anesthesiology 1996; 84: 459-471

38 Society of Gastroenterology Nurses and Associates. SGNA position statement: Statement on the use of sedation and analgesia in the gastrointestinal endoscopy setting. Gastroenterol Nurs 2003; 26: 209-211

39 Society of Gastroenterology Nurses and Associates. SGNA position statement. Statement on the use of sedation and analgesia in the gastrointestinal endoscopy setting. Gastroenterol Nurs 2004; 27: 142-144

40 Faigel DO, Baron TH, Goldstein JL et al. Guidelines for the use of deep sedation and anesthesia for GI endoscopy. Gastrointest Endosc 2002; 56: 613-617

41 American Society for gastrointestinal Endoscopy. Sedation and monitoring of patients undergoing gastrointestinal endoscopic procedures. Gastrointestinal Endoscopy 1995; 42: 626-629

42 Deutsche Gesellschaft für Anästhesiologie und Intensivmedizin. Leitlinie der Deutschen Gesellschaft für Anästhesiologie und Intensivmedizin Airway management. AWMF 2004; 45: 302-306

43 Koch DG, Arguedas MR, Fallon MB. Risk of aspiration pneumonia in suspected variceal hemorrhage: the value of prophylactic endotracheal intubation prior to endoscopy. Dig Dis Sci 2007; 52: 2225-2228

44 Rudolph SJ, Landsverk BK, Freeman ML. Endotracheal intubation for airway protection during endoscopy for severe upper GI hemorrhage. Gastrointest Endosc 2003; 57: 58-61

45 Deutsche Gesellschaft für Anästhesiologie und Intensivmedizin. Vereinbarung des Berufsverbandes Deutscher Anästhesisten und des Berufverbandes der Deutschen Chirurgen. Verantwortung für die prä-, intra- und postoperative Lagerung des Patienten. Anästh Intensivmed 1987; AWMF 65.41

46 Olithselvan A, McIntyre AS, Gorard DA. Are patients' sedation preferences at gastroscopy influenced by preceding patients' decisions? Aliment Pharmacol Ther 2004; 20: 989-992

47 Campo R, Brullet E, Montserrat A et al. Identification of factors that influence tolerance of upper gastrointestinal endoscopy. Eur J Gastroenterol Hepatol 1999; 11: 201-204

48 Subramanian S, Liangpunsakul S, Rex DK. Preprocedure patient values regarding sedation for colonoscopy. J Clin Gastroenterol 2005; 39 : 516-519

49 Abraham NS, Fallone CA, Mayrand S et al. Sedation versus no sedation in the performance of diagnostic upper gastrointestinal endoscopy: a Canadian randomized controlled cost-outcome study. Am J Gastroenterol 2004; 9: 1692-1699
50 Hedenbro JL, Ekelund M, Aberg Tet al. Oral sedation for diagnostic upper endoscopy. Endoscopy 1991; 23: 8-10

51 Kinoshita $Y$, Ishido $S$, Nishiyama $K$ et al. Arterial oxygen saturation, blood pressure, and pulse rate during upper gastrointestinal endoscopy - influence of sedation and age. J Clin Gastroenterol 1991; 13: 656-660

52 Yuno $K$, Iishi $H$, Tatsuta $M$ et al. Intravenous midazolam as a sedative for colonoscopy: a randomized, double-blind clinical trial. Aliment Pharmacol Ther 1996; 10: 981-984

53 Marriott P, Laasch HU, Wilbraham L et al. Conscious sedation for endoscopic and non-endoscopic interventional gastrointestinal procedures: meeting patients' expectations, missing the standard. Clin Radiol 2004; 59: 180-185

54 Ristikankare M, Hartikainen J, Heikkinen $M$ et al. Is routinely given conscious sedation of benefit during colonoscopy? Gastrointest Endosc 1999; 49: 566-572

55 Walmsley RS, Montgomery SM. Factors affecting patient tolerance of upper gastrointestinal endoscopy. J Clin Gastroenterol 1998; 26 : 253-255

56 Roseveare C, Seavell C, Patel P et al. Patient-controlled sedation and analgesia, using propofol and alfentanil, during colonoscopy: a prospective randomized controlled trial. Endoscopy 1998; 30: 768-773

57 Ulmer BJ, Hansen JJ, Overley CA et al. Propofol versus midazolam/fentanyl for outpatient colonoscopy: administration by nurses supervised by endoscopists. Clin Gastroenterol Hepatol 2003; 1: 425-432

58 Vargo JJ, Zuccaro Jr G, Dumot JA et al. Gastroenterologist-administered propofol versus meperidine and midazolam for advanced upper endoscopy: a prospective, randomized trial. Gastroenterology 2002; 123: $373-375$

59 Sipe BW, Rex DK, Latinovich $D$ et al. Propofol versus midazolam/meperidine for outpatient colonoscopy: administration by nurses supervised by endoscopists. Gastrointest Endosc 2002; 55: 815-825

60 Weston BR, Chadalawada $V$, Chalasani $N$ et al. Nurse-administered propofol versus midazolam and meperidine for upper endoscopy in cirrhotic patients. Am J Gastroenterol 2003; 98: 2440-2447

61 Mui LM, Teoh AY, Ng EK et al. Premedication with orally administered midazolam in adults undergoing diagnostic upper endoscopy: a double-blind placebo-controlled randomized trial. Gastrointest Endosc 2005; 61: 195-200

62 von Delius S, Hollweck R, Schmid RM et al. Midazolam-pain, but one cannot remember it: a survey among Southern German endoscopists. Eur J Gastroenterol Hepatol 2007; 19: 465-470

63 Laluna L, Allen ML, Dimarino Jr AJ. The comparison of midazolam and topical lidocaine spray versus the combination of midazolam, meperidine, and topical lidocaine spray to sedate patients for upper endoscopy. Gastrointest Endosc 2001; 53: 289-293

$64 \mathrm{Ng} \mathrm{JM}$, Kong CF, Nyam D. Patient-controlled sedation with propofol for colonoscopy. Gastrointest Endosc 2001; 54: 8-13

65 Patel S, Vargo JJ, Khandwala F et al. Deep sedation occurs frequently during elective endoscopy with meperidine and midazolam. Am J Gastroenterol 2005; 100: 2689-2695

66 Jung M, Hofmann C, Kiesslich $R$ et al. Improved sedation in diagnostic and therapeutic ERCP: propofol is an alternative to midazolam. Endoscopy 2000; 32: 233-238

67 Wehrmann T, Kokabpick H, Jacobi V et al. Long-term results of endoscopic injection of botulinum toxin in elderly achalasic patients with tortuous megaesophagus or epiphrenic diverticulum. Endoscopy 1999; 31: 352-358

68 Hofmann C, Kiesslich R, Brackertz A et al. Propofol for sedation in gastroscopy - a randomized comparison with midazolam. Z Gastroenterol 1999; 37: 589-595

69 Koshy G, Nair S, Norkus EP et al. Propofol versus midazolam and meperidine for conscious sedation in GI endoscopy. Am J Gastroenterol 2000; 95: 1476-1479

70 Reimann FM, Samson U, Derad I et al. Synergistic sedation with lowdose midazolam and propofol for colonoscopies. Endoscopy 2000; 32: 239-244

71 Heuss LT, Schnieper P, Drewe J et al. Conscious sedation with propofol in elderly patients: a prospective evaluation. Aliment Pharmacol Ther 2003; 17: 1493-1501

72 Kazama T, Takeuchi K, Ikeda Ket al. Optimal propofol plasma concentration during upper gastrointestinal endoscopy in young, middleaged, and elderly patients. Anesthesiology 2000; 93: 662-669 
73 Rex DK, Overley CA, Walker J. Registered nurse-administered propofol sedation for upper endoscopy and colonoscopy: Why? When? How? Rev Gastroenterol Disord 2003; 3: 70-80

74 Gottschling $S$, Larsen $R$, Meyer $S$ et al. Acute pancreatitis induced by short-term propofol administration. Paediatr Anaesth 2005; 15: 1006-1008

75 Jawaid Q, Presti ME, Neuschwander-Tetri BA et al. Acute pancreatitis after single-dose exposure to propofol: a case report and review of literature. Dig Dis Sci 2002; 47: 614-618

76 Fodale V, La Monaca E. Propofol infusion syndrome: an overview of a perplexing disease. Drug Saf 2008; 31: 293-303

77 Kulling D, Fantin AC, Biro P et al. Safer colonoscopy with patient-controlled analgesia and sedation with propofol and alfentanil. Gastrointest Endosc 2001; 54: 1-7

78 Bright E, Roseveare C, Dalgleish D et al. Patient-controlled sedation for colonoscopy: a randomized trial comparing patient-controlled administration of propofol and alfentanil with physician-administered midazolam and pethidine. Endoscopy 2003; 35: 683-687

79 Lee DW, Chan AC, Sze TS et al. Patient-controlled sedation versus intravenous sedation for colonoscopy in elderly patients: a prospective randomized controlled trial. Gastrointest Endosc 2002; 56: 629-632

80 Crepeau T, Poincloux L, Bonny C et al. Significance of patient-controlled sedation during colonoscopy. Results from a prospective randomized controlled study. Gastroenterol Clin Biol 2005; 29: 1090-1096

81 Lee DW, Chan AC, Wong SK et al. The safety, feasibility, and acceptability of patient-controlled sedation for colonoscopy:prospective study. Hong Kong Med J 2004; 10: 84-88

82 Egan TD, Kern SE, Johnson KB et al. The pharmacokinetics and pharmacodynamics of propofol in a modified cyclodextrin formulation (Captisol) versus propofol in a lipid formulation (Diprivan): an electroencephalographic and hemodynamic study in a porcine model. Anesth Analg 2003; 97: 72-79

83 Fanti L, Agostoni M, Arcidiacono PG et al. Target-controlled infusion during monitored anesthesia care in patients undergoing EUS: propofol alone versus midazolam plus propofol. A prospective doubleblind randomised controlled trial. Dig Liver Dis 2007; 39: 81-86

84 Leslie K, Absalom A, Kenny GN. Closed loop control of sedation for colonoscopy using the Bispectral Index. Anaesthesia 2002; 57: 693-697

85 Campbell L, Imrie G, Doherty P et al. Patient maintained sedation for colonoscopy using a target controlled infusion of propofol. Anaesthesia 2004; 59: 127-132

86 Gillham MJ, Hutchinson RC, Carter $R$ et al. Patient-maintained sedation for ERCP with a target-controlled infusion of propofol: a pilot study. Gastrointest Endosc 2001; 54: 14-17

87 Stonell CA, Leslie K, Absalom AR. Effect-site targeted patient-controlled sedation with propofol: comparison with anaesthetist administration for colonoscopy. Anaesthesia 2006; 61: 240-247

88 Pambianco J, Meroric J, Martin R. Feasibility assesment of computer assisted personalized sedation: a sedation delivery system to administer propofol for gastrointestinal endoscopy. Gastrointestinal Endoscopy 2006; 63 (5): AB 189

89 Reves JG, Fragen RJ, Vinik HR et al. Midazolam: pharmacology and uses. Anesthesiology 1985; 62: 310-324

90 Donnelly MB, Scott WA, Daly DS. Sedation for upper gastrointestinal endoscopy: a comparison of alfentanil-midazolam and meperidinediazepam. Can J Anaesth 1994; 41: 1161-1165

91 Ginsberg GG, Lewis JH, Gallagher JE et al. Diazepam versus midazolam for colonoscopy: a prospective evaluation of predicted versus actual dosing requirements. Gastrointest Endosc 1992; 38: 651-656

92 Macken E, Gevers AM, Hendrickx A et al. Midazolam versus diazepam in lipid emulsion as conscious sedation for colonoscopy with or without reversal of sedation with flumazenil. Gastrointest Endosc 1998 47: $57-61$

93 Lavies NG, Creasy T, Harris K et al. Arterial oxygen saturation during upper gastrointestinal endoscopy: influence of sedation and operator experience. Am J Gastroenterol 1988; 83: 618-622

94 Carrougher JG, Kadakia S, Shaffer RT et al. Venous complications of midazolam versus diazepam. Gastrointest Endosc 1993; 39: 396-399

95 Ladas SD, Aabakken L, Rey JF et al. Use of sedation for routine diagnostic upper gastrointestinal endoscopy: a European Society of Gastrointestinal Endoscopy Survey of National Endoscopy Society Members. Digestion 2006; 74: 69-77
96 Zakko SF, Seifert HA, Gross JB. A comparison of midazolam and diazepam for conscious sedation during colonoscopy in a prospective double-blind study. Gastrointest Endosc 1999; 49: 684-689

97 Cantor DS, Baldridge ET. Premedication with meperidine and diazepam for upper gastrointestinal endoscopy precludes the need for topical anesthesia. Gastrointest Endosc 1986; 32: 339-341

98 Lee MG, Hanna $W$, Harding $H$. Sedation for upper gastrointestinal endoscopy: a comparative study of midazolam and diazepam. Gastrointest Endosc 1989; 35: 82-84

99 Lader M, Morton S. Benzodiazepine problems. Br J Addict 1991; 86: 823-828

100 Christe C, Janssens JP, Armenian B et al. Midazolam sedation for upper gastrointestinal endoscopy in older persons: a randomized, doubleblind, placebo-controlled study. J Am Geriatr Soc 2000; 48: 1398-1403

101 Froehlich F, Schwizer W, Thorens J et al. Conscious sedation for gastroscopy: patient tolerance and cardiorespiratory parameters. Gastroenterology 1995; 108: 697-704

102 Froehlich $F$, Thorens J, Schwizer W et al. Sedation and analgesia for colonoscopy: patient tolerance, pain, and cardiorespiratory parameters. Gastrointest Endosc 1997; 45: 1-9

103 Yano $H$, Iishi H, Tatsuta $M$ et al. Oxygen desaturation during sedation for colonoscopy in elderly patients. Hepatogastroenterology 1998; 45: 2138-2141

104 Hirsh I, Vaissler A, Chernin J et al. Fentanyl or tramadol, with midazolam, for outpatient colonoscopy: analgesia, sedation, and safety. Dig Dis Sci 2006; 51: 1946-1951

105 Lee SY, Son HJ, Lee JM et al. Identification of factors that influence conscious sedation in gastrointestinal endoscopy. J Korean Med Sci 2004; 19: 536-540

106 Morrow JB, Zuccaro Jr G, Conwell DL et al. Sedation for colonoscopy using a single bolus is safe, effective, and efficient: a prospective, randomized, double-blind trial. Am J Gastroenterol 2000; 95: 2242-2247

107 Terruzzi V, Meucci G, Radaelli F et al. Routine versus „on demand“ sedation and analgesia for colonoscopy: a prospective randomized controlled trial. Gastrointest Endosc 2001; 54: 169-174

108 Kankaria A, Lewis JH, Ginsberg G et al. Flumazenil reversal of psychomotor impairment due to midazolam or diazepam for conscious sedation for upper endoscopy. Gastrointest Endosc 1996; 44: 416-421

109 Saletin M, Malchow $H$, Muhlhofer $H$ et al. A randomised controlled trial to evaluate the effects of flumazenil after midazolam premedication in outpatients undergoing colonoscopy. Endoscopy 1991; 23: 331-333

110 Mora CT, Torjman $M$, White PF. Sedative and ventilatory effects of midazolam infusion: effect of flumazenil reversal. Can J Anaesth 1995 ; 42: 677-684

111 Carter AS, Bell GD, Coady Tet al. Speed of reversal of midazolam-induced respiratory depression by flumazenil - a study in patients undergoing upper G.I.endoscopy. Acta Anaesthesiol Scand 1990; Suppl 92: 59-64

112 Andrews PJ, Wright DJ, Lamont MC. Flumazenil in the outpatient. A study following midazolam as sedation for upper gastrointestinal endoscopy. Anaesthesia 1990; 45: 445-448

113 Bartelsman JF, Sars PR, Tytgat GN. Flumazenil used for reversal of midazolam-induced sedation in endoscopy outpatients. Gastrointest Endosc 1990; 36: S9-S12

114 Norton AC, Dundas CR. Induction agents for day-case anaesthesia. A double- blind comparison of propofol and midazolam antagonised by flumazenil. Anaesthesia 1990; 45: 198-203

115 Wehrmann T, Grotkamp J, Stergiou N et al. Electroencephalogram monitoring facilitates sedation with propofol for routine ERCP: a randomized, controlled trial. Gastrointest Endosc 2002; 56: 817-824

116 Meining A, Semmler V, Kassem AM et al. The effect of sedation on the quality of upper gastrointestinal endoscopy: an investigator-blinded, randomized study comparing propofol with midazolam. Endoscopy 2007; 39: 345-349

117 Hansen JJ, Ulmer BJ, Rex DK. Technical performance of colonoscopy in patients sedated with nurse-administered propofol. Am J Gastroenterol 2004; 99: 52-56

118 Rudner R, Jalowiecki P, Kawecki P et al. Conscious analgesia/sedation with remifentanil and propofol versus total intravenous anesthesia with fentanyl, midazolam, and propofol for outpatient colonoscopy. Gastrointest Endosc 2003; 57: 657-663 
119 Qadeer MA, Vargo JJ, Khandwala F et al. Propofol versus traditional sedative agents for gastrointestinal endoscopy: a meta-analysis. Clin Gastroenterol Hepatol 2005; 3: 1049-1056

120 Krugliak P, Ziff B, Rusabrov Y et al. Propofol versus midazolam for conscious sedation guided by processed EEG during endoscopic retrograde cholangiopancreatography: a prospective, randomized, double-blind study. Endoscopy 2000; 32: 677-682

121 Riphaus A, Stergiou N, Wehrmann T. Sedation with propofol for routine ERCP in high-risk octogenarians: a randomized, controlled study. Am J Gastroenterol 2005; 100: 1957-1963

122 Wehrmann T, Riphaus A. Sedation with propofol for interventional endoscopic procedures: A risk factor analysis. Scand J Gastroenterol 2007; 10: 1-7

123 Ishiguro T, Ishiguro C, Ishiguro $G$ et al. Midazolam sedation for upper gastrointestinal endoscopy: comparison between the states of patients in partial and complete amnesia. Hepatogastroenterology 2002; 49: 438-440

124 Arrowsmith JB, Gerstman BB, Fleischer DE et al. Results from the American Society for Gastrointestinal Endoscopy/U.S. Food and Drug Administration collaborative study on complication rates and drug use during gastrointestinal endoscopy. Gastrointest Endosc 1991; 37: 421-427

125 Basu S, Krishnamurthy B, Walsh TH. Value of fentanyl in flexible sigmoidoscopy. World J Surg 2004; 28: 930-934

126 Ishido S, Kinoshita Y, Kitajima $N$ et al. Fentanyl for sedation during upper gastrointestinal endoscopy. Gastrointest Endosc 1992; 38 : 689-692

127 Akcaboy ZN, Akcaboy EY, Albayrak D et al. Can remifentanil be a better choice than propofol for colonoscopy during monitored anesthesia care? Acta Anaesthesiol Scand 2006; 50: 736-741

128 Green SM, Li J. Ketamine in adults: what emergency physicians need to know about patient selection and emergence reactions. Acad Emerg Med 2000; 7: 278-281

129 White PF, Way WL, Trevor AJ. Ketamine - its pharmacology and therapeutic uses. Anesthesiology 1982; 56: 119-136

130 Aggarwal A, Ganguly S, Anand VK et al. Efficacy and safety of intravenous ketamine for sedation and analgesia during pediatric endoscopic procedures. Indian Pediatr 1998; 35: 1211-1214

131 Gilger MA, Spearman RS, Dietrich CL et al. Safety and effectiveness of ketamine as a sedative agent for pediatric GI endoscopy. Gastrointest Endosc 2004; 59: 659-663

132 Rosing C, Trinkl W, Rosing $K$ et al. Midazolam plus ketamine as premedication for colonoscopies. A double-blind, randomized study. Med Klin 1991; 86: 190-193

133 Ong WC, Santosh D, Lakhtakia $S$ et al. A randomized controlled trial on use of propofol alone versus propofol with midazolam, ketamine, and pentazocine „sedato-analgesic cocktail“ for sedation during ERCP. Endoscopy 2007; 39: 807-812

134 Saunders BP, Fukumoto M, Halligan S et al. Patient-administered nitrous oxide/oxygen inhalation provides effective sedation and analgesia for colonoscopy. Gastrointest Endosc 1994; 40: 418-421

135 Forbes GM, Collins BJ. Nitrous oxide for colonoscopy: a randomized controlled study. Gastrointest Endosc 2000; 51: 271-277

136 Maslekar SK, Hughes M, Skinn E et al. Randomised controlled trial of sedation for colonoscopy:entonox versus intravenous sedation. Gastrointestinal Endoscopy 2006; 63 (5): AB97-AB 97 S

137 Mui WL, Kwong WH, Li AC et al. Premedication with intravenous ketorolac trometamol (Toradol) in colonoscopy: a randomized controlled trial. Am J Gastroenterol 2005; 100: 2669-2673

138 Lieberman DA, Wuerker CK, Katon RM. Cardiopulmonary risk of esophagogastroduodenoscopy. Role of endoscope diameter and systemic sedation. Gastroenterology 1985; 88: 468-472

139 Mizuno J, Matsuki M, Gouda Y et al. Sedation with intravenous midazolam during upper gastrointestinal endoscopy - changes in hemodynamics, oxygen saturation and memory. Masui 2003; 52: 976-980

140 Oei-Lim VL, Kalkman CJ, Makkes PC et al. Patient-controlled versus anesthesiologist-controlled conscious sedation with propofol for dental treatment in anxious patients. Anesth Analg 1998; 86: 967-972

141 Ristikankare $M$, Julkunen $R$, Heikkinen $M$ et al. Sedation, topical pharyngeal anesthesia and cardiorespiratory safety during gastroscopy. J Clin Gastroenterol 2006; 40: 899-905

142 Boldy DA, Lever LR, Unwin PR et al. Sedation for endoscopy: midazolam or diazepam and pethidine? Br J Anaesth 1988; 61: 698-701
143 Milligan KR, Howe JP, McLoughlin J et al. Midazolam sedation for outpatient fibreoptic endoscopy: evaluation of alfentanil supplementation. Ann R Coll Surg Engl 1988; 70: 304-306

144 Radaelli F, Meucci G, Terruzzi V et al. Single bolus of midazolam versus bolus midazolam plus meperidine for colonoscopy: a prospective, randomized, double- blind trial. Gastrointest Endosc 2003; 57: 329-335

145 Paspatis GA, Manolaraki M, Xirouchakis G et al. Synergistic sedation with midazolam and propofol versus midazolam and pethidine in colonoscopies: a prospective, randomized study. Am J Gastroenterol 2002; 97: 1963-1967

146 Seifert H, Schmitt TH, Gultekin Tet al. Sedation with propofol plus midazolam versus propofol alone for interventional endoscopic procedures: a prospective, randomized study. Aliment Pharmacol Ther 2000; 14: 1207-1214

147 DiPalma JA, Herrera JL, Weis FR et al. Alfentanil for conscious sedation during colonoscopy. South Med J 1995; 88: 630-634

148 Moerman AT, Struys MM, Vereecke HE et al. Remifentanil used to supplement propofol does not improve quality of sedation during spontaneous respiration. J Clin Anesth 2004; 16: 237-243

149 Rembacken BJ, Axon AT. The role of pethidine in sedation for colonoscopy. Endoscopy 1995; 27: 244-247

150 VanNatta ME, Rex DK. Propofol alone titrated to deep sedation versus propofol in combination with opioids and/or benzodiazepines and titrated to moderate sedation for colonoscopy. Am J Gastroenterol 2006; 101: 2209-2217

151 Cordruwisch $W$, Doroschko $M$, Wurbs D. Deep sedation in gastrointestinal endoscopic interventions: safety and reliability of a combination of midazolam and propofol. Dtsch Med Wochenschr 2000; 125: 619-622

152 Mui LM, Ng EK, Chan KC et al. Randomized, double-blinded, placebocontrolled trial of intravenously administered hyoscine N-butyl bromide in patients undergoing colonoscopy with patient-controlled sedation. Gastrointest Endosc 2004; 59: 22-27

153 Vargo JJ, Holub JL, Faigel DO et al. Risk factors for cardiopulmonary events during propofol-mediated upper endoscopy and colonoscopy. Aliment Pharmacol Ther 2006; 24: 955-963

154 Iber FL, Sutberry M, Gupta R et al. Evaluation of complications during and after conscious sedation for endoscopy using pulse oximetry. Gastrointest Endosc 1993; 39: 620-625

155 Thompson AM, Park KG, Kerr F et al. Safety of fibreoptic endoscopy: analysis of cardiorespiratory events. Br J Surg 1992; 79: 1046-1049

156 Assy N, Rosser BG, Grahame GR et al. Risk of sedation for upper GI endoscopy exacerbating subclinical hepatic encephalopathy in patients with cirrhosis. Gastrointest Endosc 1999; 49: 690-694

157 Vasudevan AE, Goh KL, Bulgiba AM. Impairment of psychomotor responses after conscious sedation in cirrhotic patients undergoing therapeutic upper GI endoscopy. Am J Gastroenterol 2002; 97: 1717-1721

158 Muravchick S. Anesthesia for the geriatric patient. Clinical Anesthesia 2001: 1205-1216

159 Qureshi WA, Zuckerman MJ, Adler DG et al. ASGE guideline: modifications in endoscopic practice for the elderly. Gastrointest Endosc 2006; 63: 566-569

160 Darling E. Practical considerations in sedating the elderly. Crit Care Nurs Clin North Am 1997; 9: 371-380

161 Dhariwal A, Plevris JN, Lo NT et al. Age, anemia, and obesity-associated oxygen desaturation during upper gastrointestinal endoscopy. Gastrointest Endosc 1992; 38: 684-688

162 Scholer SG, Schafer DF, Potter JF. The effect of age on the relative potency of midazolam and diazepam for sedation in upper gastrointestinal endoscopy. J Clin Gastroenterol 1990; 12: 145-147

163 Hamdy NA, Kennedy HJ, Nicholl J et al. Sedation for gastroscopy: a comparative study of midazolam and Diazemuls in patients with and without cirrhosis. Br J Clin Pharmacol 1986; 22: 643-647

164 Riphaus A, Lechowicz I, Porth J et al. Sedierung mit Propofol für endoskopische Interventionen bei Patienten mit Leberzirrhose. Ein alternatives Verfahren, um die Exacerbation einer subklinischen hepatischen Encephalopathie zu verhindern. Eine randomisiert-kontrollierte Studie. Z- Gastroenterol 2007; 45: 784

165 Heuss LT, Schnieper P, Drewe J et al. Safety of propofol for conscious sedation during endoscopic procedures in high-risk patients-a prospective, controlled study. Am J Gastroenterol 2003; 98: 1751-1757 
166 Harikumar R, Raj M, Paul A et al. Listening to music decreases need for sedative medication during colonoscopy: a randomized, controlled trial. Indian J Gastroenterol 2006; 25: 3-5

167 Rudin D, Kiss A, Wetz RV et al. Music in the endoscopy suite: a metaanalysis of randomized controlled studies. Endoscopy 2007; 39: 507-510

168 Aisenberg J, Cohen LB. Sedation in endoscopic practice. Gastrointest Endosc Clin N Am 2006; 16: 695-708

169 American Medical Association. The use of pulse oximetry during conscious sedation. Council on Scientific Affairs. JAMA 1993; 270: 1463-1468

170 Conigliaro $R$, Rossi A. Implementation of sedation guidelines in clinical practice in Italy: results of a prospective longitudinal multicenter study. Endoscopy 2006; 38: 1137-1143

171 Eisenbacher $S$, Heard L. Capnography in the gastroenterology lab. Gastroenterol Nurs 2005; 28: 99-105

172 Graber $R G$. Propofol in the endoscopy suite: an anesthesiologist's perspective. Gastrointest Endosc 1999; 49: 803-806

173 Harris JK, Froehlich F, Wietlisbach V et al. Factors associated with the technical performance of colonoscopy: An EPAGE Study. Dig Liver Dis 2007; 39: 678-689

174 Jamieson J. Anesthesia and sedation in the endoscopy suite? (Influences and options). Curr Opin Anaesthesiol 1999; 12: 417-423

175 Kulling D, Orlandi $M$, Inauen $W$. Propofol sedation during endoscopic procedures: how much staff and monitoring are necessary? Gastrointest Endosc 2007; 66: 443-449

176 Leslie K, Stonell CA. Anaesthesia and sedation for gastrointestinal endoscopy. Curr Opin Anaesthesiol 2005; 18: 431-436

177 Lopez RL. Subcomite de Protocolos Of The Spanish Society Of Gastrointestinal Endoscopy Seed. Sedation/analgesia guidelines for endoscopy. Rev Esp Enferm Dig 2006; 98: 685-692

178 Mahoney LB, Lightdale JR. Sedation of the Pediatric and Adolescent Patient for GI Procedures. Curr Treat Options Gastroenterol 2007; 10: $412-421$

179 Moos DD, Cuddeford JD. Methemoglobinemia and benzocaine. Gastroenterol Nurs 2007; 30: 342-345

180 Morlote EB, Zweng TN, Strodel WE. Hemodynamic monitoring and pulse oximetry during percutaneous gastrostomy and jejunostomy: necessity or nuisance? Surg Endosc 1991; 5: 130-134

181 Overley $C A, \operatorname{Rex} D K$. A nursing perspective on sedation and nurse-administered propofol for endoscopy. Gastrointest Endosc Clin N Am 2004; 14: 325-333

182 Robbertze R, Posner KL, Domino KB. Closed claims review of anesthesia for procedures outside the operating room. Curr Opin Anaesthesiol 2006; 19: 436-442

183 Society of Gastroenterology Nurses and Associates. Guidelines for nursing care of the patient receiving sedation and analgesia in the gastrointestinal endoscopy setting. Gastroenterol Nurs 1997; 20 (2): Suppl 1-6

184 American Society for Gastrointestinal Endoscopy. Training guideline for use of propofol in gastrointestinal endoscopy. Gastrointest Endosc 2004; 60 (2): 167-172

185 Vargo JJ, Ahmad AS, Aslanian HR et al. Training in patient monitoring and sedation and analgesia. Gastrointest Endosc 2007; 66: 7-10

186 Zebris J, Maurer W. Quality assurance in the endoscopy suite: sedation and monitoring. Gastrointest Endosc Clin N Am 2004; 14: 415-429

187 Silvermann W, Chotiprasidhi R, Chuttani J et al. Monitoring equipment for endoscopy. Gastrointestinal Endoscopy 2003; 59 (7): 761-765

188 Kiesslich R, Moenk S, Reinhardt Ket al. Combined simulation training: a new concept and workshop is useful for crisis management in gastrointestinal endoscopy. Z Gastroenterol 2005; 43: 1031-1039

189 Sieg A. Propofol sedation in outpatient colonoscopy by trained practice nurses supervised by the gastroenterologist: a prospective evaluation of over 3000 cases. Z Gastroenterol 2007; 45: 697-701

190 Heuss LT, Froehlich F, Beglinger C. Changing patterns of sedation and monitoring practice during endoscopy: results of a nationwide survey in Switzerland. Endoscopy 2005; 37: 161-166

191 Froehlich F, Harris JK, Wietlisbach V et al. Current sedation and monitoring practice for colonoscopy: an International Observational Study (EPAGE). Endoscopy 2006; 38: 461-469

192 Anderson JL, Junkins E, Pribble C et al. Capnography and depth of sedation during propofol sedation in children. Ann Emerg Med 2007; 49: 9-13
193 Levine DA, Platt SL. Novel monitoring techniques for use with procedural sedation. Curr Opin Pediatr 2005; 17: 351-354

194 Soto RG, Fu ES, Vila Jr H et al. Capnography accurately detects apnea during monitored anesthesia care. Anesth Analg 2004; 99: 379-382, table

195 Vargo JJ, Zuccaro Jr G, Dumot JA et al. Automated graphic assessment of respiratory activity is superior to pulse oximetry and visual assessment for the detection of early respiratory depression during therapeutic upper endoscopy. Gastrointest Endosc 2002; 55: 826-831

196 Koniaris LG, Wilson S, Drugas G et al. Capnographic monitoring of ventilatory status during moderate (conscious) sedation. Surg Endosc 2003; 17: 1261-1265

197 Chen SC, Rex DK. An initial investigation of bispectral monitoring as an adjunct to nurse-administered propofol sedation for colonoscopy. Am J Gastroenterol 2004; 99: 1081-1086

198 Drake LM, Chen SC, Rex DK. Efficacy of bispectral monitoring as an adjunct to nurse-administered propofol sedation for colonoscopy: a randomized controlled trial. Am J Gastroenterol 2006; 101: 2003-2007

199 Parzreller M, Wenk M. Aufklärung und Einwilligung bei ärztlichen Eingriffen. Deutsches Ärzteblatt 2007; 109: 576-586

200 Christopher J. Gastrointestinal Bleeding in the Elderly Patient. Am J Gastroenterol 2000; 95: 590-595

201 Hochberger J, Maiss J, Hahn EG. The use of simulators for training in GI endoscopy. Endoscopy 2002; 34: 727-729

202 Rieger H.-J. Zur Rechtzeitigkeit der Patientenaufklärung. Dtsch Med Wochschr 2003; 128: 1728-1729

203 Sieg A, Hachmoeller-Eisenbach $U$, Eisenbach T. Prospective evaluation of complications in outpatient GI endoscopy: a survey among German gastroenterologists. Gastrointest Endosc 2001; 53: 620-627

204 Mahajan RJ, Johnson JC, Marshall JB. Predictors of patient cooperation during gastrointestinal endoscopy. J Clin Gastroenterol 1997; 24: 220-223

205 Pena LR, Mardini HE, Nickl NJ. Development of an instrument to assess and predict satisfaction and poor tolerance among patients undergoing endoscopic procedures. Dig Dis Sci 2005; 50: 1860-1871

206 Lazzaroni M, Bianchi PG. Preparation, premedication, and surveillance. Endoscopy 2005; 37: 101-109

207 Laufs A, Uhlenbruck W. Handbuch des Arztrechts 2002; 3. Aufl

208 Med R41 - 42 OLG Stuttgart, 1986

209 MedR407 - 408 OLG Zweibrücken, 1995

210 Hochberger J. Die Einverständniserklärung zu endoskopischen Eingriffen. In: Sauerbruch T, Scheurlen C (Hrsg). Empfehlungen der Deutschen Gesellschaft für Verdauungs- und Stoffwechselkrankheiten (DGVS) zur Durchführung endoskopischer Untersuchungen. Stuttgart: Demter Verlag, 2002; 3. erweiterte und überarbeitete Aufl: $1-11$

211 Hayes A, Buffum M. Educating patients after conscious sedation for gastrointestinal procedures. Gastroenterol Nurs 2001; 24: 54-57

212 Smith MR, Bell GD, Fulton B et al. A comparison of winged steel needles and Teflon cannulas in maintaining intravenous access during gastrointestinal endoscopy. Gastrointest Endosc 1993; 39: 33-36

213 Alcain G, Guillen P, Escolar A et al. Predictive factors of oxygen desaturation during upper gastrointestinal endoscopy in nonsedated patients. Gastrointest Endosc 1998; 48: 143-147

214 Sieg A, Theilmeier A. Results of coloscopy screening in 2005 - an Internet-based documentation. Dtsch Med Wochenschr 2006; 131: 379-383

215 Jurell KR, O'Connor KW, Slack J et al. Effect of supplemental oxygen on cardiopulmonary changes during gastrointestinal endoscopy. Gastrointest Endosc 1994; 40: 665-670

216 Reshef $R$, Shiller $M$, Kinberg $R$ et al. A prospective study evaluating the usefulness of continuous supplemental oxygen in various endoscopic procedures. Isr J Med Sci 1996; 32: 736-740

217 Wang CY, Ling LC, Cardosa MS et al. Hypoxia during upper gastrointestinal endoscopy with and without sedation and the effect of preoxygenation on oxygen saturation. Anaesthesia 2000; 55: 654-658

218 Ristikankare M, Julkunen R, Mattila $M$ et al. Conscious sedation and cardiorespiratory safety during colonoscopy. Gastrointest Endosc 2000; 52: 48-54

219 Petelenz M, Gonciarz M, Macfarlane P et al. Sympathovagal balance fluctuates during colonoscopy. Endoscopy 2004; 36: 508-514 
220 Bhalla A, Sood A, Sachdeva A et al. Cardiorespiratory compromise under conscious sedation during upper gastrointestinal endoscopy. J Coll Physicians Surg Pak 2006; 16 (9): 585-589

221 Lewalter T. Notfall Herzrhythmusstörungen. Deutsches Ärzteblatt 2007; 104 (17): 1172-1180

222 Leslie K, Tay T, Neo E. Intravenous fluid to prevent hypotension in patients undergoing elective colonoscopy. Anaesth Intensive Care 2006; 34: 316-321

223 Ristikankare M, Julkunen R, Laitinen Tet al. Effect of conscious sedation on cardiac autonomic regulation during colonoscopy. Scand J Gastroenterol 2000; 35: 990-996

224 Sieg A, Hachmoeller-Eisenbach U, Heisenbach T. How safe is premedication in ambulatory endoscopy in Germany.A prospective study in gastroenterology specialty practices. Dtsch Med Wochenschr 2000; 125: $1288-1293$

225 Royal College of Surgeons of England Commission on the Provision of Surgical Services. Report of the working party on guidelines for sedation by non- anesthetists. 1993. www.rcseng.ac.uk/fds/clinical_guidelines-31k

226 Aldrete JA. The post-anesthesia recovery score revisited. J Clin Anesth 1995; 7: 89-91
227 Willey J, Vargo JJ, Connor JT et al. Quantitative assessment of psychomotor recovery after sedation and analgesia for outpatient EGD. Gastrointest Endosc 2002; 56: 810-816

228 Nuotto EJ, Korttila KT, Lichtor JL et al. Sedation and recovery of psychomotor function after intravenous administration of various doses of midazolam and diazepam. Anesth Analg 1992; 74: 265-271

229 Thapar P, Zacny JP, Thompson $W$ et al. Using alcohol as a standard to assess the degree of impairment induced by sedative and analgesic drugs used in ambulatory surgery. Anesthesiology 1995; 82: 53-59

230 Korttila K, Linnoila M. Psychomotor skills related to driving after intramuscular administration of diazepam and meperidine. Anesthesiology 1975; 125: 619-622

231 Korttila K, Linnoila M. Recovery and skills related to driving after intravenous sedation: dose-response relationship with diazepam. $\mathrm{Br} \mathrm{J}$ Anaesth 1975; 47: 457-463

232 Korttila K. Recovery after intravenous sedation. A comparison of clinical and paper and pencil tests used in assessing late effects of diazepam. Anaesthesia 1976; 31: 724-731

233 Piletti R, Davis PJ, Redlinger $R$ et al. Effect on hospital wide sedation practice after Implentation of the 2001 JCAHO procedural sedation and analgesia guidelines. Arch Adolsec Med 2006; 160: 211-216 